\title{
1993 lower Cook Inlet geologic field program
}

Amoco Oil Co.

GMC DATA REPORT 453

This GMC data report from the Amoco Heritage collection has been made available through funding from the FY2018 USGS National Geological and Geophysical Data Preservation Program, Grant Number G18AP00054. This project report is presented in its original format and has not been reviewed for technical content or for conformity to the editorial standards of DGGS. It should not be used or cited as reviewed data.

2019

State of Alaska

Department of Natural Resources

Division of Geological \& Geophysical Surveys

GEOLOGIC MATERIALS CENTER
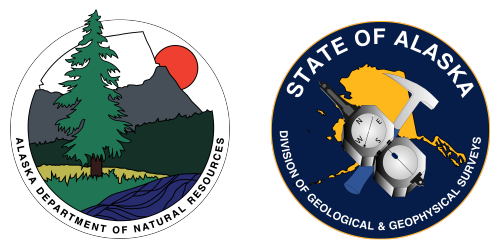
1993--Lower Cook Inlet

Geologic Field Program

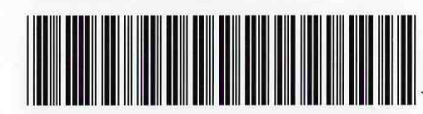

GMC-000082542 


\begin{tabular}{|c|c|c|c|c|c|}
\hline SECTION NAME & CODE & TRS & QUAD & DATE & MEASURED BY \\
\hline Douglas River & 93 KAT 102 & TBA & TBA & $8 / 9 / 93$ & GFH, Data entered on computer by MAC \\
\hline & & & & & \\
\hline DEPTH & GR1 & GR2 & GR3 & GR AVE. & LITHOLOGY \\
\hline 0 & 1687 & 1685 & 1710 & 1694.00 & Mudstone \\
\hline 5 & 1137 & 1095 & 1128 & 1120.00 & fg ss \\
\hline 10 & 1456 & 1505 & 1521 & 1494.00 & slts \\
\hline 15 & 1416 & 1334 & 1458 & 1402.67 & slts as above \\
\hline 20 & 1370 & 1349 & 1402 & 1373.67 & slts as above \\
\hline 25 & 1538 & 1598 & 1421 & 1519.00 & slts as above \\
\hline 30 & 1506 & 1541 & 1473 & 1506.67 & slts as above \\
\hline 35 & 1344 & 1331 & 1270 & 1315.00 & vfg ss \\
\hline 40 & 1397 & 1338 & 1326 & 1353.67 & vfg ss a.a., ash beds \\
\hline 45 & 1462 & 1498 & 1433 & 1464.33 & slts \\
\hline 50 & 1233 & 1261 & 1257 & 1250.33 & slts a.a. \\
\hline 55 & 1410 & 1344 & 1292 & 1348.67 & slts a.a. \\
\hline 60 & 1344 & 1301 & 1254 & 1299.67 & slts a.a. \\
\hline 65 & 1244 & 1233 & 1201 & 1226.00 & sits a.a. \\
\hline 70 & 1233 & 1225 & 1267 & 1241.67 & slts a.a. \\
\hline 75 & 1429 & 1469 & 1430 & 1442.67 & slts a.a. \\
\hline 80 & 1121 & 1102 & 1065 & 1096.00 & fg ss \\
\hline 85 & 0 & 0 & 0 & 0.00 & covered section \\
\hline 90 & 1564 & 1540 & 1561 & 1555.00 & slts \\
\hline 95 & 1468 & 1558 & 1491 & 1505.67 & slts a.a. \\
\hline 100 & 1586 & 1623 & 1610 & 1606.33 & slts a.a. \\
\hline 105 & 1581 & 1515 & 1616 & 1570.67 & slts a.a. \\
\hline 110 & 1608 & 1612 & 1620 & 1613.33 & slts a.a. \\
\hline 115 & 1550 & 1490 & 1549 & 1529.67 & slts a.a. \\
\hline 120 & 1614 & 1655 & 1684 & 1651.00 & slts a.a. \\
\hline 125 & 0 & 0 & 0 & 0.00 & No gamma ray obtained \\
\hline 130 & 0 & 0 & 0 & 0.00 & No gamma ray obtained \\
\hline
\end{tabular}

Page 1 


\begin{tabular}{|c|c|c|c|c|c|}
\hline 135 & 0 & 0 & 0 & 0.00 & No gamma ray obtained \\
\hline 140 & 0 & 0 & 0 & 0.00 & No gamma ray obtained \\
\hline 145 & 0 & 0 & 0 & 0.00 & No gamma ray obtained \\
\hline 150 & 0 & 0 & 0 & 0.00 & No gamma ray obtained \\
\hline 155 & 0 & 0 & 0 & 0.00 & No gamma ray obtained \\
\hline 160 & 0 & 0 & 0 & 0.00 & No gamma ray obtained \\
\hline 165 & 0 & 0 & 0 & 0.00 & No gamma ray obtained \\
\hline 170 & 0 & 0 & 0 & 0.00 & No gamma ray obtained \\
\hline 175 & 0 & 0 & 0 & 0.00 & No gamma ray obtained \\
\hline 180 & 0 & 0 & 0 & 0.00 & No gamma ray obtained \\
\hline 185 & 0 & 0 & 0 & 0.00 & No gamma ray obtained \\
\hline 190 & 0 & 0 & 0 & 0.00 & No gamma ray obtained \\
\hline 195 & 0 & 0 & 0 & 0.00 & No gamma ray obtained \\
\hline 200 & 0 & 0 & 0 & 0.00 & No gamma ray obtained \\
\hline 205 & 0 & 0 & 0 & 0.00 & No gamma ray obtained \\
\hline 210 & 0 & 0 & 0 & 0.00 & No gamma ray obtained \\
\hline 215 & 0 & 0 & 0 & 0.00 & No gamma ray obtained \\
\hline 220 & 0 & 0 & 0 & 0.00 & No gamma ray obtained \\
\hline 225 & 0 & 0 & 0 & 0.00 & No gamma ray obtained \\
\hline 230 & 0 & 0 & 0 & 0.00 & No gamma ray obtained \\
\hline 235 & 0 & 0 & 0 & 0.00 & No gamma ray obtained \\
\hline 240 & 0 & 0 & 0 & 0.00 & No gamma ray obtained \\
\hline 245 & 0 & 0 & 0 & 0.00 & No gamma ray obtained \\
\hline 250 & 0 & 0 & 0 & 0.00 & No gamma ray obtained \\
\hline 255 & 0 & 0 & 0 & 0.00 & No gamma ray obtained \\
\hline 260 & 0 & 0 & 0 & 0.00 & No gamma ray obtained \\
\hline 265 & 0 & 0 & 0 & 0.00 & No gamma ray obtained \\
\hline 270 & 0 & 0 & 0 & 0.00 & No gamma ray obtained \\
\hline 275 & 0 & 0 & 0 & 0.00 & No gamma ray obtained \\
\hline 280 & 1586 & 1652 & 1668 & 1635.33 & slts \\
\hline 285 & 1460 & 1425 & 1495 & 1460.00 & slts \\
\hline 290 & 1683 & 1653 & 1678 & 1671.33 & Mud rich slts \\
\hline 295 & 1654 & 1675 & 1684 & 1671.00 & Mud rich slts a.a. \\
\hline
\end{tabular}


Gamma Ray 93 KAT 102

\begin{tabular}{|c|c|c|c|c|c|}
\hline 300 & 1480 & 1407 & 1404 & 1430.33 & slts \\
\hline 305 & 1583 & 1628 & 1671 & 1627.33 & slts a.a. \\
\hline 310 & 0 & 0 & 0 & 0.00 & Covered section \\
\hline 315 & 0 & 0 & 0 & 0.00 & Covered section \\
\hline 320 & 1550 & 1548 & 1587 & 1561.67 & sits \\
\hline 325 & 1551 & 1585 & 1530 & 1555.33 & slts a.a. \\
\hline 330 & 1664 & 1648 & 1641 & 1651.00 & Flaggy slty mdst \\
\hline 335 & 1496 & 1544 & 1517 & 1519.00 & slts \\
\hline 340 & 1517 & 1601 & 1501 & 1539.67 & slts a.a. \\
\hline 345 & 1461 & 1446 & 1447 & 1451.33 & vfg ss/slts \\
\hline 350 & 1510 & 1589 & 1570 & 1556.33 & slts \\
\hline 355 & 1972 & 1938 & 1881 & 1930.33 & Highly horizontally bioturb. slts \\
\hline 360 & 1608 & 1609 & 1673 & 1630.00 & sits \\
\hline 365 & 1512 & 1629 & 1605 & 1582.00 & slts a.a. \\
\hline 375 & 1289 & 1474 & 1436 & 1399.67 & slts a.a. \\
\hline 380 & 1504 & 1460 & 1521 & 1495.00 & slts a.a. \\
\hline 385 & 1628 & 1604 & 1695 & 1642.33 & slts a.a. \\
\hline 390 & 1424 & 1435 & 1391 & 1416.67 & v. f. ss, more fossils \\
\hline 395 & 1444 & 1469 & 1465 & 1459.33 & v. f. ss, more fossils a.a. \\
\hline 400 & 1630 & 1608 & 1608 & 1615.33 & sltst \\
\hline 405 & 1464 & 1467 & 1445 & 1458.67 & slst a.a. \\
\hline 410 & 1243 & 1249 & 1236 & 1242.67 & v.f. ss \\
\hline 415 & 1334 & 1304 & 1348 & 1328.67 & v.f. ss a.a. \\
\hline 420 & 1579 & 1597 & 1582 & 1586.00 & slst \\
\hline 425 & 1373 & 1366 & 1372 & 1370.33 & slst \\
\hline 430 & 1535 & 1530 & 1562 & 1542.33 & slst \\
\hline 435 & 1550 & 1494 & 1551 & 1531.67 & slst \\
\hline 440 & 1324 & 1338 & 1340 & 1334.00 & v. f. ss \\
\hline 445 & 1435 & 1527 & 1522 & 1494.67 & slst \\
\hline 450 & 1336 & 1391 & 1314 & 1347.00 & v. f. ss \\
\hline 455 & 1735 & 1699 & 1680 & 1704.67 & slst \\
\hline 460 & 1412 & 1478 & 1487 & 1459.00 & slst \\
\hline 465 & 1518 & 1466 & 1436 & 1473.33 & slst \\
\hline
\end{tabular}


Gamma Ray 93 KAT 102

\begin{tabular}{|l|l|l|l|l|l|}
\hline 470 & 1348 & 1367 & 1395 & 1370.00 & v. f. ss \\
\hline 475 & 1325 & 1339 & 1340 & 1334.67 & slst \\
\hline 480 & 1624 & 1547 & 1560 & 1577.00 & slst \\
\hline 485 & 1487 & 1489 & 1512 & 1496.00 & slst \\
\hline 490 & 1425 & 1491 & 1423 & 1446.33 & slst \\
\hline 495 & 1524 & 1536 & 1532 & 1530.67 & slst \\
\hline 500 & 1572 & 1591 & 1542 & 1568.33 & slst \\
\hline 505 & 1601 & 1595 & 1575 & 1590.33 & slst \\
\hline 510 & 1614 & 1580 & 1580 & 1591.33 & slst \\
\hline 515 & 1748 & 1758 & 1796 & 1767.33 & slst, near ash \\
\hline 520 & 1318 & 1360 & 1334 & 1337.33 & slst \\
\hline 525 & 1470 & 1459 & 1492 & 1473.67 & slst \\
\hline 530 & 1231 & 1279 & 1192 & 1234.00 & v.f. ss \\
\hline 535 & 1442 & 1402 & 1444 & 1429.33 & slst \\
\hline 540 & 1264 & 1179 & 1238 & 1227.00 & slst \\
\hline
\end{tabular}




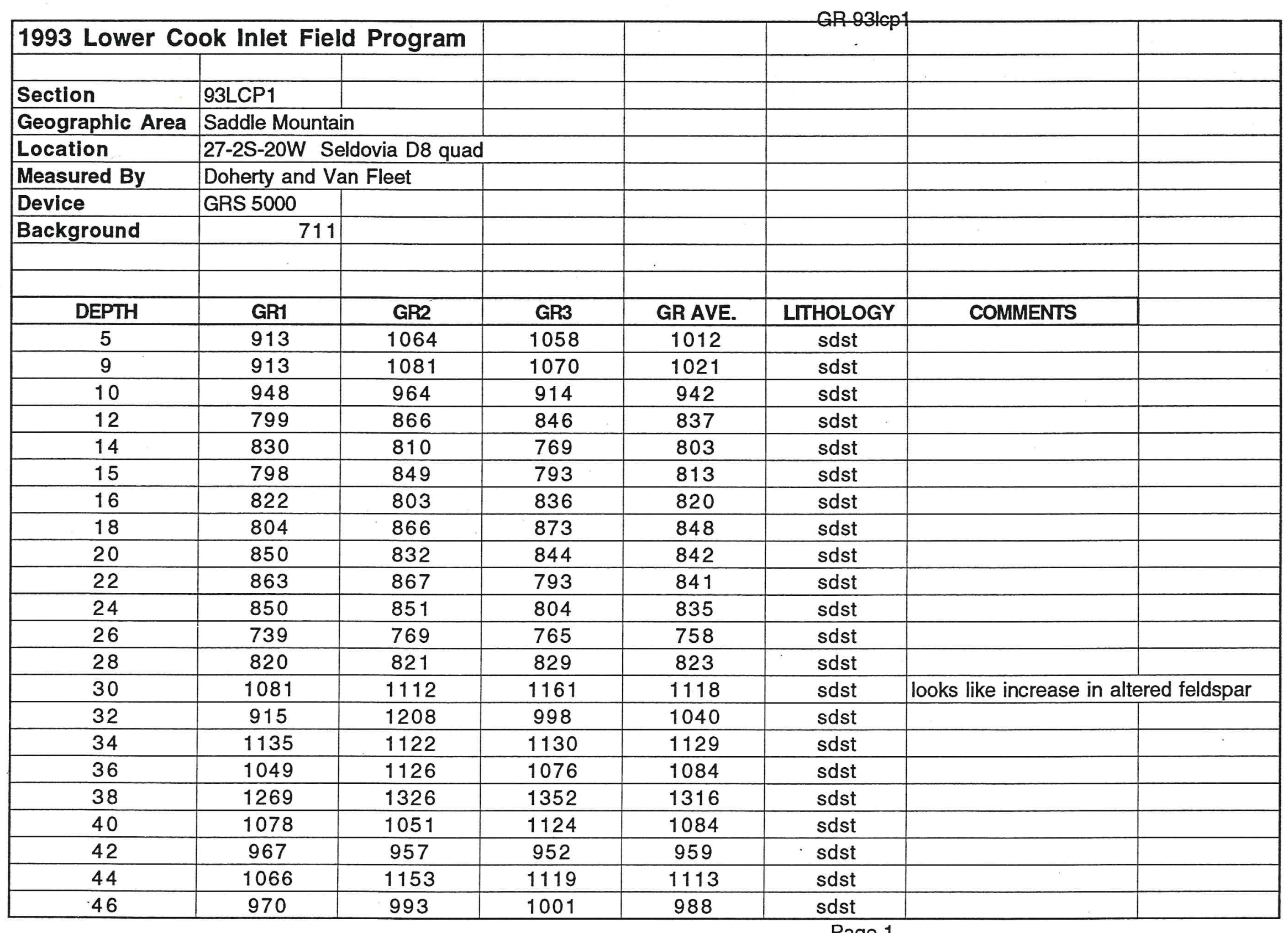




\begin{tabular}{|c|c|c|c|c|c|c|c|}
\hline 48 & 1006 & 999 & 1092 & 1032 & sdst & & \\
\hline 50 & 1127 & 1128 & 1092 & 1116 & sdst & & \\
\hline 52 & 1262 & 1189 & 1242 & 1231 & sdst & & \\
\hline 54 & 1007 & 982 & 996 & 995 & sdst & & \\
\hline 56 & 1008 & 1015 & 1020 & 1014 & sdst & & \\
\hline 58 & 900 & 927 & 874 & 900 & sdst & & \\
\hline 60 & 974 & 965 & 911 & 950 & sdst & & \\
\hline 62 & 1058 & 1081 & 1112 & 1084 & sdst & & \\
\hline 64 & 1121 & 1079 & 1148 & 1116 & sdst & & \\
\hline 66 & 978 & 975 & 1057 & 1003 & sdst & & \\
\hline 68 & 1084 & 1304 & 1260 & 1216 & sdst & & \\
\hline 70 & 1704 & 1683 & 1675 & 1687 & sdst & & \\
\hline 72 & 1188 & 1151 & 1174 & 1171 & sdst & & \\
\hline 74 & 1669 & 1657 & 1619 & 1648 & sdst & & \\
\hline 76 & 1868 & 1882 & 1857 & 1869 & sdst & & \\
\hline 78 & 1498 & 1422 & 1424 & 1448 & sdst & & \\
\hline
\end{tabular}




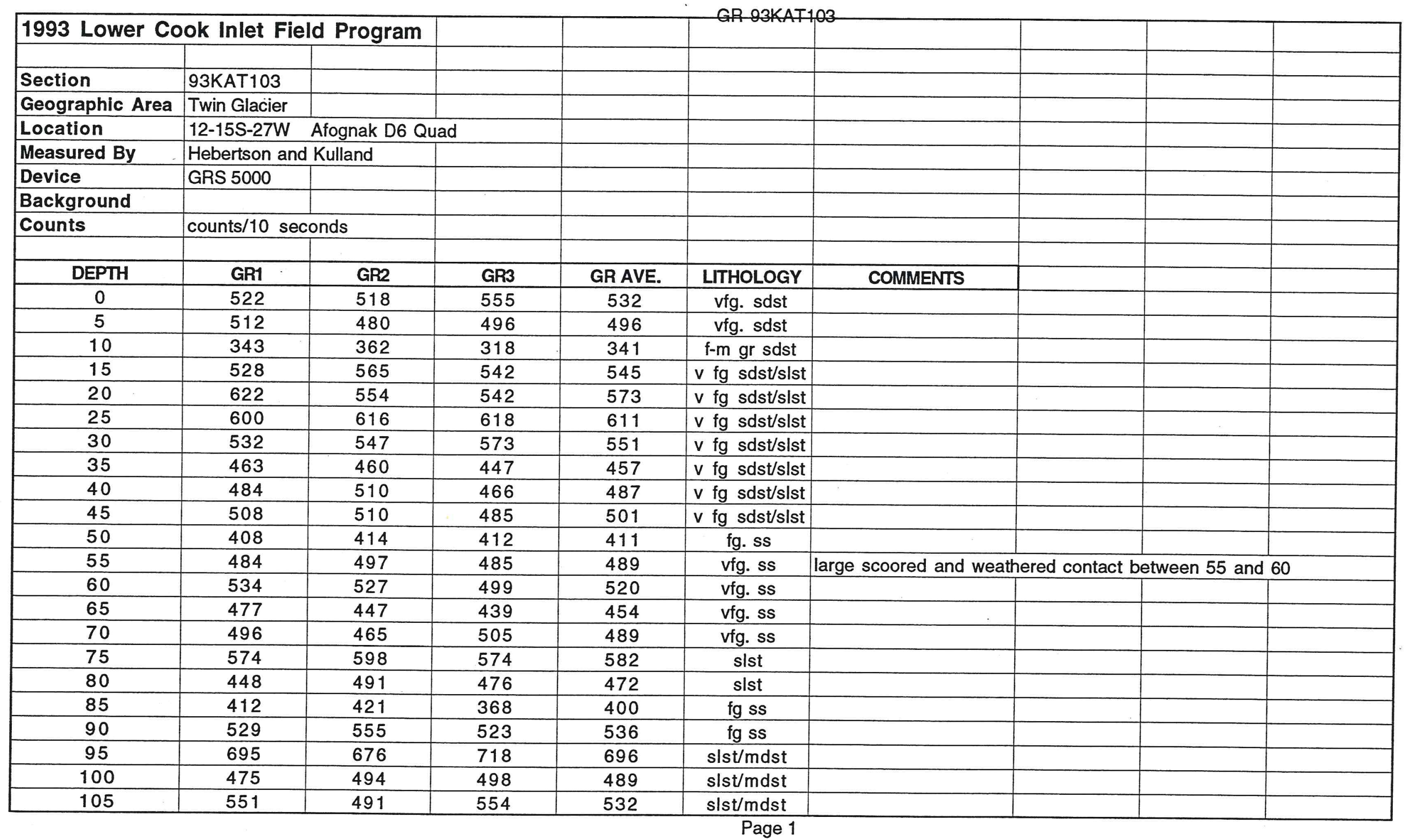




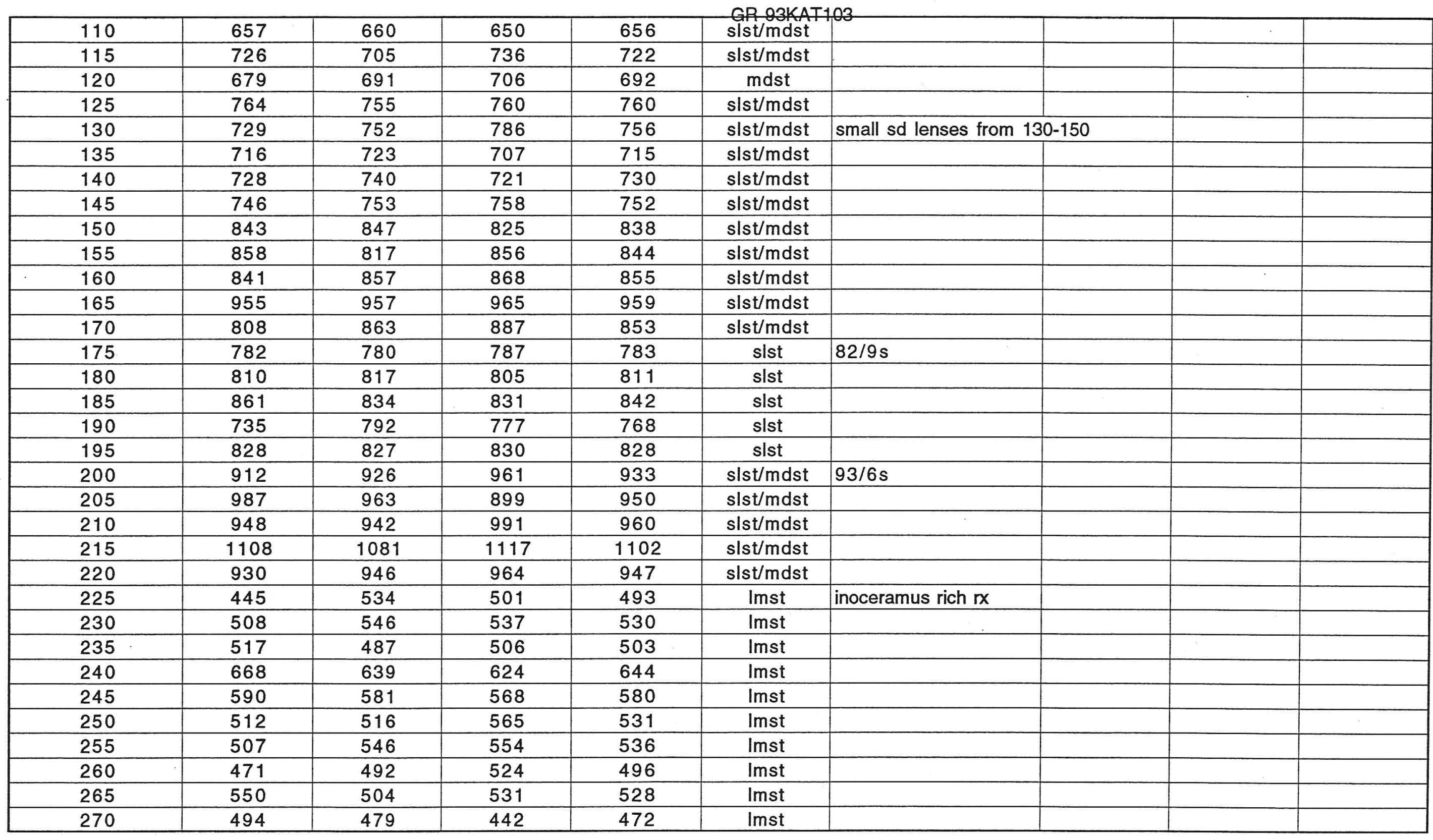

Page 2 


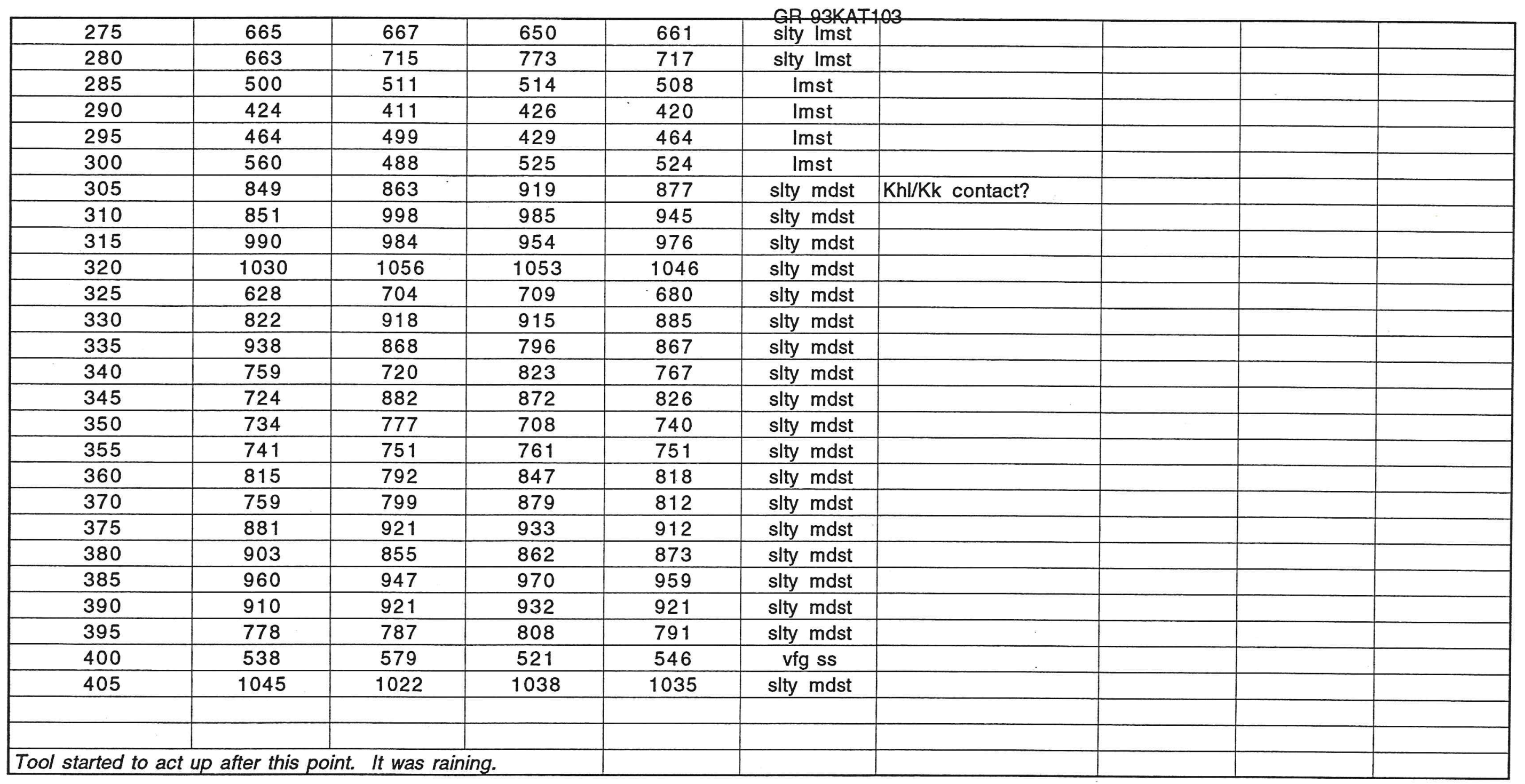

Page 3 


\begin{tabular}{|c|c|c|c|c|c|c|}
\hline SECTION NAME & CODE & TRS & QUAD & DATE & MEASUREDBY & INSTRUMENT \\
\hline HALLO GLACIER & 93KAT104-A & SW/NE 32-20S-30W & MT. KATMAI B1 & 32735.00 & RK, JVF & SCINTEX GIS-5 \\
\hline & & & & & & \\
\hline & & & & & & \\
\hline DEPTH & GR1 & GR2 & GR3 & GR AVE. & LITHOLOGY & COMMENTS \\
\hline 0 & 1111 & 1124 & 1089 & 1108.00 & SLTST & dip: 13 deg., S5E, observed conj. shear sets. \\
\hline 5 & 948 & 961 & 915 & 941.33 & SLTST & \\
\hline 10 & 1189 & 1171 & 1138 & 1166.00 & SLTST & \\
\hline 20 & 1019 & 1013 & 1035 & 1022.33 & SLTST & \\
\hline 25 & 1079 & 1044 & 1090 & 1071.00 & SLTST & acute angle conjugate shears $\mathrm{S} 20 \mathrm{~W}$ \\
\hline 30 & 1201 & 1304 & 1344 & 1283.00 & SLTST & \\
\hline 35 & 1030 & 998 & 990 & 1006.00 & SLTST & \\
\hline 40 & 1023 & 1000 & 1045 & 1022.67 & SLTST & conjugate shears S4OE \\
\hline 45 & 1019 & 1060 & 1082 & 1053.67 & SLTST & \\
\hline 50 & 1004 & 955 & 950 & 969.67 & SLTST & conjugate shears due $\mathrm{S}$ \\
\hline 55 & 1022 & 1075 & 1005 & 1034.00 & SLTST & \\
\hline 60 & 998 & 1008 & 1024 & 1010.00 & SLTST & conjugate shears $\mathrm{S} 10 \mathrm{E}$ \\
\hline 65 & 1017 & 962 & 1020 & 999.67 & SLTST & conjugate shears $\mathrm{S} 5 \mathrm{E}$, adjacent $8^{\prime \prime}$ dike \\
\hline 70 & 977 & 988 & 989 & 984.67 & SLTST & dike as above \\
\hline 75 & 997 & 967 & 951 & 971.67 & SLTST & vertical fractures \\
\hline 80 & 1072 & 1081 & 1084 & 1079.00 & SLTST & \\
\hline 85 & 960 & 1055 & 985 & 1000.00 & SLTST & \\
\hline 90 & 1176 & 1141 & 1100 & 1139.00 & SLTST & conjugate shears S20E \\
\hline 95 & 1018 & 988 & 986 & 997.33 & SLTST & end of transect A. $100^{\prime}$ estimated missing section \\
\hline 195 & 1489 & 1487 & 1516 & 1497.33 & SLTST & begin transect B, dip 17 deg., S14E \\
\hline 200 & 1484 & 1429 & 1467 & 1460.00 & SLTST & \\
\hline 205 & 1371 & 1352 & 1412 & 1378.33 & SLTST & \\
\hline 210 & 1360 & 1296 & 1240 & 1298.67 & SLTST & conjugate shears S10E \\
\hline 215 & 1370 & 1317 & 1302 & 1329.67 & SLTST & \\
\hline 220 & 1367 & 1465 & 1480 & 1437.33 & SLTST & \\
\hline 225 & 1434 & 1439 & 1424 & 1432.33 & SLTST & densely fractured, blocky \\
\hline 230 & 1240 & 1212 & 1266 & 1239.33 & SLTST & densely fractured, blocky \\
\hline
\end{tabular}

Page 1 


\begin{tabular}{|c|c|c|c|c|c|c|}
\hline 235 & 1278 & 1254 & 1317 & 1283.00 & SLTST & densely fractured, blocky \\
\hline 240 & 1167 & 1124 & 1164 & 1151.67 & SLTST & densely fractured, blocky \\
\hline 245 & 648 & 608 & 588 & 614.67 & SLTST & dike \\
\hline 250 & 1203 & 1194 & 1211 & 1202.67 & SLTST & fractures parallel to dip \\
\hline 255 & 1271 & 1252 & 1301 & 1274.67 & SLTST & densely fractured, blocky \\
\hline 260 & 1235 & 1255 & 1325 & 1271.67 & SLTST & densely fractured, blocky \\
\hline 265 & 1378 & 1293 & 1364 & 1345.00 & SLTST & dip 16 degrees S20E \\
\hline 270 & 1534 & 1571 & 1567 & 1557.33 & SLTST & densely fractured, blocky \\
\hline 275 & 1297 & 1321 & 1315 & 1311.00 & SLTST & densely fractured, blocky \\
\hline 280 & 1307 & 1423 & 1398 & 1376.00 & SLTST & densely fractured, blocky \\
\hline 285 & 1230 & 1264 & 1336 & 1276.67 & SLTST & densely fractured, blocky \\
\hline 290 & 1439 & 1396 & 1456 & 1430.33 & SLTST & densely fractured, blocky \\
\hline 295 & 1303 & 1266 & 1251 & 1273.33 & SLTST & densely fractured, blocky \\
\hline 300 & 1327 & 1120 & 1222 & 1223.00 & SLTST & densely fractured, blocky \\
\hline 305 & 1229 & 1222 & 1255 & 1235.33 & SLTST & densely fractured, blocky \\
\hline 310 & 1428 & 1304 & 1293 & 1341.67 & SLTST & densely fractured, blocky \\
\hline 315 & 1304 & 1366 & 1288 & 1319.33 & SLTST & densely fractured, blocky \\
\hline 320 & 1310 & 1333 & 1303 & 1315.33 & SLTST & densely fractured, blocky \\
\hline 325 & 1406 & 1436 & 1413 & 1418.33 & SLTST & densely fractured, blocky \\
\hline 330 & 1168 & 1236 & 1212 & 1205.33 & SLTST & densely fractured, blocky \\
\hline 335 & 1224 & 1230 & 1305 & 1253.00 & SLTST & dip 15 degrees due $S$ \\
\hline 340 & 1301 & 1385 & 1335 & 1340.33 & SLTST & densely fractured, blocky \\
\hline 345 & 1226 & 1241 & 1185 & 1217.33 & SLTST & densely fractured, blocky \\
\hline 350 & 1447 & 1381 & 1382 & 1403.33 & SLTST & weathers splintery-blocky \\
\hline 355 & 1206 & 1214 & 1255 & 1225.00 & SLTST & densely fractured, blocky \\
\hline 360 & & & & 0.00 & & covered section \\
\hline 365 & 1420 & 1372 & 1345 & 1379.00 & SLTST & densely fractured, blocky \\
\hline 370 & 1330 & 1325 & 1360 & 1338.33 & SLTST & densely fractured, blocky \\
\hline 377 & 1365 & 1308 & 1371 & 1348.00 & SLTST & densely fractured, blocky \\
\hline 380 & 1072 & 1130 & 1147 & 1116.33 & SLTST & densely fractured, blocky \\
\hline 386 & 1371 & 1325 & 1256 & 1317.33 & SLTST & densely fractured, blocky \\
\hline 390 & 1409 & 1369 & 1345 & 1374.33 & SLTST & densely fractured, blocky \\
\hline 400 & 1267 & 1197 & 1271 & 1245.00 & SLTST & dip 17 degrees S3E \\
\hline
\end{tabular}

Page 2 


\begin{tabular}{|l|l|l|l|l|l|l|}
\hline 405 & 1142 & 1165 & 1147 & 1151.33 & SLTST & densely fractured, blocky \\
\hline 410 & 1327 & 1349 & 1356 & 1344.00 & SLTST & densely fractured, blocky \\
\hline 415 & 1119 & 1116 & 1174 & 1136.33 & SLTST & densely fractured, blocky \\
\hline & & & & 0.00 & & \\
\hline & & & & 0.00 & & \\
\hline & & & & 0.00 & & \\
\hline & & & & 0.00 & & \\
\hline & & & & 0.00 & & \\
\hline
\end{tabular}




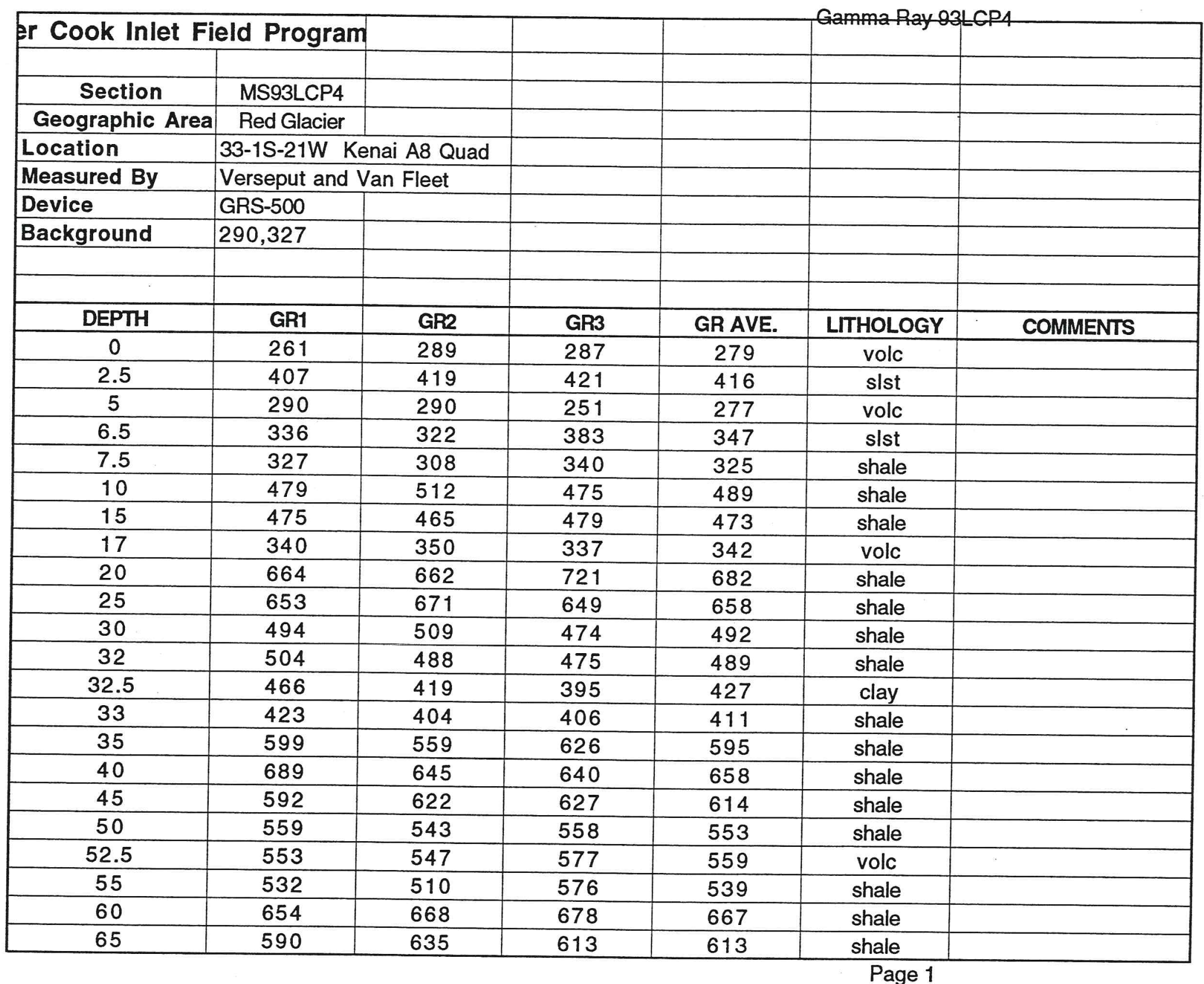




\begin{tabular}{|c|c|c|c|c|c|c|}
\hline 68 & 314 & 309 & 308 & 310 & volc sd & \\
\hline 70 & 282 & 309 & 285 & 292 & volc sd & \\
\hline 71 & 370 & 386 & 359 & 372 & volc sd & \\
\hline 75 & 660 & 684 & 710 & 685 & shale & \\
\hline 77.5 & 481 & 449 & 470 & 467 & alt volc & \\
\hline 80 & 621 & 593 & 603 & 606 & shale & \\
\hline 85 & 664 & 607 & 611 & 627 & shale & \\
\hline 90 & 584 & 587 & 545 & 572 & shale & \\
\hline 92.5 & 313 & 320 & 324 & 319 & volc sd & \\
\hline 95 & 672 & 640 & 694 & 669 & shale & \\
\hline 96 & 544 & 521 & 518 & 528 & sd & \\
\hline 100 & 659 & 645 & 695 & 666 & shale & \\
\hline 102 & 528 & 526 & 561 & 538 & shale & \\
\hline 105 & 550 & 578 & 621 & 583 & shale & \\
\hline 110 & 634 & 573 & 582 & 596 & sd & \\
\hline 113.5 & 477 & 533 & 473 & 494 & shale & \\
\hline 115 & 646 & 567 & 612 & 608 & shale & \\
\hline 120 & 577 & 557 & 500 & 545 & shale & \\
\hline 125 & 554 & 536 & 514 & 535 & shale & \\
\hline 130 & 535 & 510 & 506 & 517 & shale & \\
\hline 135 & 529 & 543 & 509 & 527 & shale & \\
\hline 140 & 441 & 443 & 441 & 442 & sd & \\
\hline 145 & 465 & 505 & 466 & 479 & shale & \\
\hline 150 & 564 & 588 & 563 & 572 & shale & \\
\hline 155 & 543 & 591 & 583 & 572 & shale & \\
\hline 160 & 590 & 599 & 637 & 609 & shale & \\
\hline 165 & 623 & 642 & 584 & 616 & shale & \\
\hline 170 & 791 & 833 & 797 & 807 & shale & \\
\hline 172.5 & 586 & 598 & 587 & 590 & sd & \\
\hline 175 & 711 & 717 & 724 & 717 & shale & \\
\hline 178 & 546 & 559 & 555 & 553 & sd & \\
\hline 180 & 613 & 665 & 656 & 645 & shale & \\
\hline 184 & 652 & 642 & 670 & 655 & shale & \\
\hline
\end{tabular}




\begin{tabular}{|c|c|c|c|c|c|c|}
\hline 185 & 636 & 614 & 666 & 639 & $\begin{array}{c}\text { Gamma pay } 93 \\
\text { shale }\end{array}$ & $6+44$ \\
\hline 190 & 594 & 610 & 596 & 600 & shale & \\
\hline 195 & 554 & 570 & 559 & 561 & shale & \\
\hline 200 & 570 & 571 & 595 & 579 & shale & \\
\hline 202.5 & 470 & 488 & 490 & 483 & shale & \\
\hline 205 & 632 & 633 & 636 & 634 & shale & \\
\hline \multirow[t]{4}{*}{210} & 535 & 522 & 501 & 519 & shale & \\
\hline & & & & \#DIV/O! & & \\
\hline & & & & \#DIV/O! & & \\
\hline & & & & \#DIV/0! & & \\
\hline
\end{tabular}

Page 3 


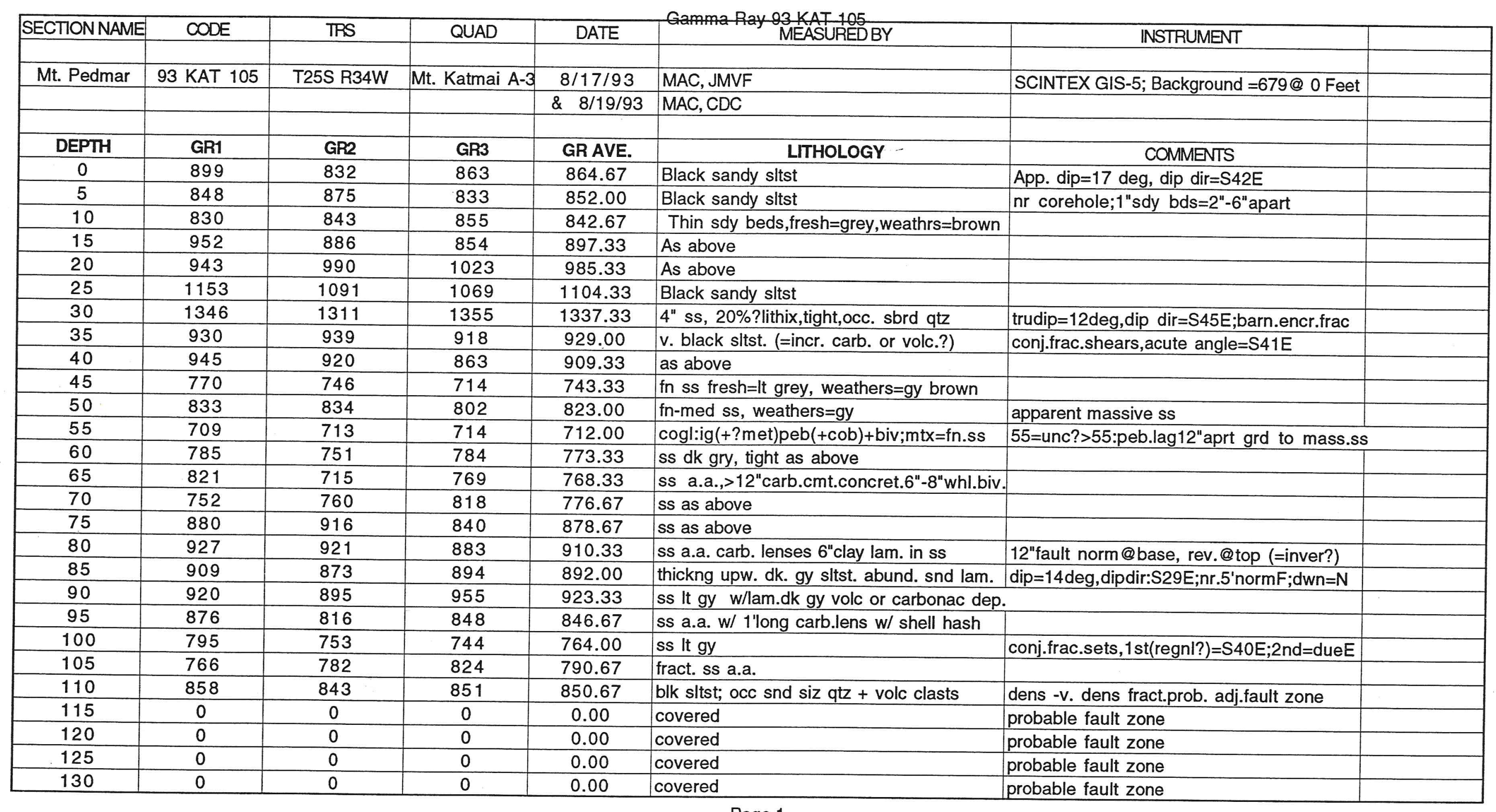

Page 1 


\begin{tabular}{|c|c|c|c|c|c|c|}
\hline 135 & 0 & 0 & 0 & 0.00 & covered & probable fault zone \\
\hline 140 & 0 & 0 & 0 & 0.00 & covered & probable fault zone \\
\hline 145 & 0 & 0 & 0 & 0.00 & covered & probable fault zone \\
\hline 150 & 0 & 0 & 0 & 0.00 & covered & probable fault zone \\
\hline 155 & 0 & 0 & 0 & 0.00 & covered & probable fault zone \\
\hline 160 & 1437 & 1475 & 1465 & 1459.00 & sltst w/concrts:weathr gr/blk-rust=volc? & dip=8.5 deg, dipdir131deg; Bckgrd=724@ @ 160 \\
\hline 165 & 1472 & 1504 & 1515 & 1497.00 & sltst a.a. & \\
\hline 170 & 1589 & 1542 & 1513 & 1548.00 & sltst weathers gy; densely fractured & unit more brittle than units above \&below it \\
\hline 175 & 1141 & 1119 & 1163 & 1141.00 & sltst more silty than units above, bioturb. & dip=11deg; dip dir=149deg \\
\hline 180 & 1089 & 1124 & 1081 & 1098.00 & v.fn.ss abund cly incl; $>1$ 'diam.limey concrt & \\
\hline 185 & 0 & 0 & 0 & 0.00 & No reading/ lithol: $185-190$ due to fault & \\
\hline 195 & 1043 & 1068 & 1040 & 1050.33 & sdy sltst & \\
\hline 200 & 996 & 970 & 1012 & 992.67 & sdy sltst a.a. & \\
\hline 205 & 807 & 869 & 908 & 861.33 & lam. fn ss w/ concret, bedng intensly fract & fract due to poss fault zone (covered) \\
\hline 210 & 1699 & 1698 & 1713 & 1703.33 & dk. gy. blocky slst & \\
\hline 215 & 1304 & 1244 & 1344 & 1297.33 & a.a. & \\
\hline 220 & 1362 & 1375 & 1420 & 1385.67 & a.a. & \\
\hline 225 & 1758 & 1781 & 1852 & 1797.00 & a.a. & \\
\hline 230 & 1410 & 1395 & 1458 & 1421.00 & slst w/ dk gy volc or organic rich deposits & \\
\hline 235 & 1118 & 1107 & 1144 & 1123.00 & a.a. & \\
\hline 240 & 1042 & 1068 & 1026 & 1045.33 & gy slst & \\
\hline 245 & 988 & 1024 & 973 & 995.00 & f-med ss $w /$ concretions $6^{\prime \prime}->12^{\prime \prime}$ diam & \\
\hline 250 & 1100 & 1117 & 1132 & 1116.33 & gy slst & \\
\hline 255 & 1239 & 1160 & 1223 & 1207.33 & gy slst blocky & \\
\hline 260 & 1096 & 1077 & 1063 & 1078.67 & gy slst & \\
\hline 265 & 987 & 1147 & 1167 & 1100.33 & gy f ss w/ Inoc shells & \\
\hline 270 & 1077 & 1078 & 1188 & 1114.33 & f. ss \&slst w/ Inoc shells & \\
\hline 275 & 1160 & 1292 & 1226 & 1226.00 & a.a. + concretions & Fault zone strike slip no vertical displacment \\
\hline 280 & 1236 & 1210 & 1120 & 1188.67 & gy slst w/gy concr(cob sze)\&wht 1 "concr & \\
\hline 285 & 1126 & 1078 & 1128 & 1110.67 & gy slst \& minor fine ss & \\
\hline 290 & 992 & 1017 & 1056 & 1021.67 & gy slst & 2 rev. fault down to $\mathrm{N}$ \\
\hline 295 & 1038 & 978 & 990 & 1002.00 & gy f ss \& sltst & \\
\hline 300 & 885 & 1039 & 991 & 971.67 & gy f-med ss & \\
\hline
\end{tabular}

Page 2 


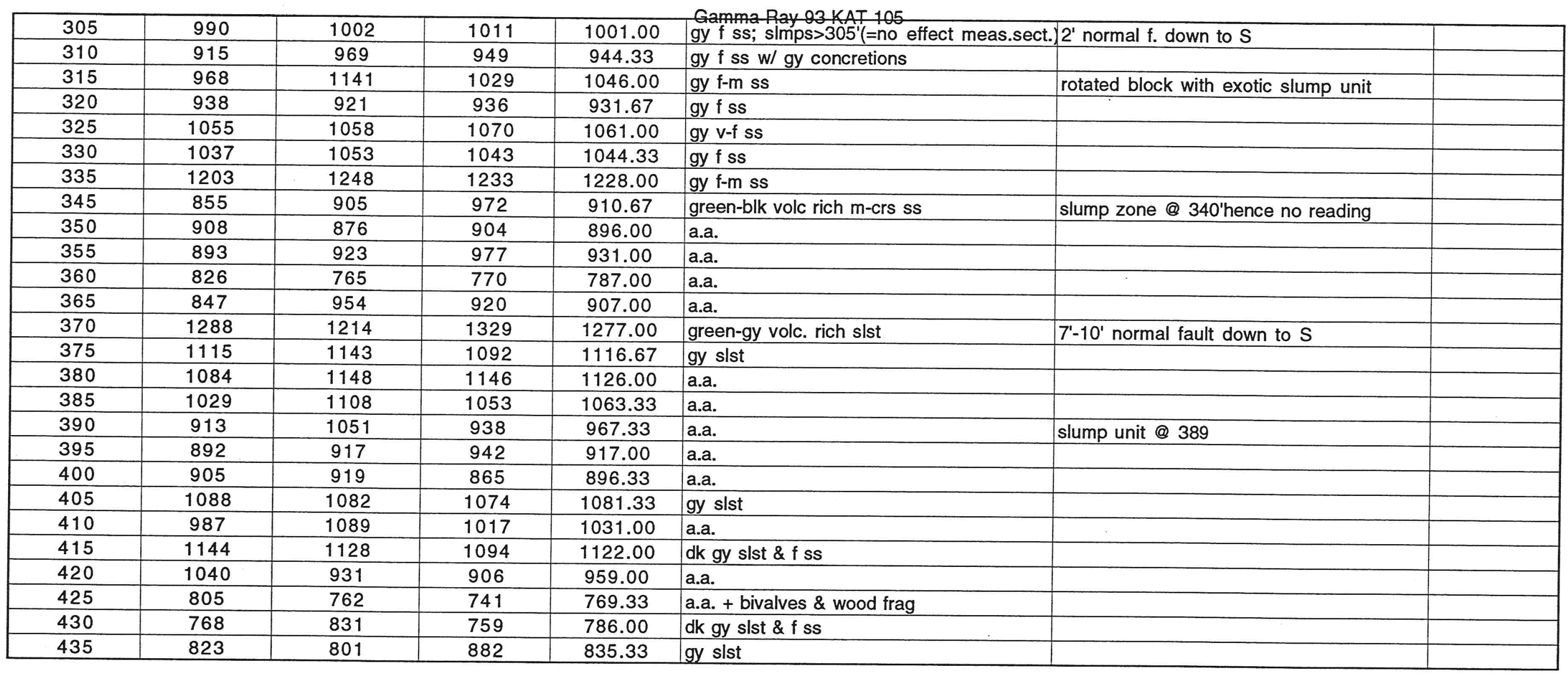


TABLE 1

Milligal Values for LaCoste \& Romberg Model G Gravity Meter \#30

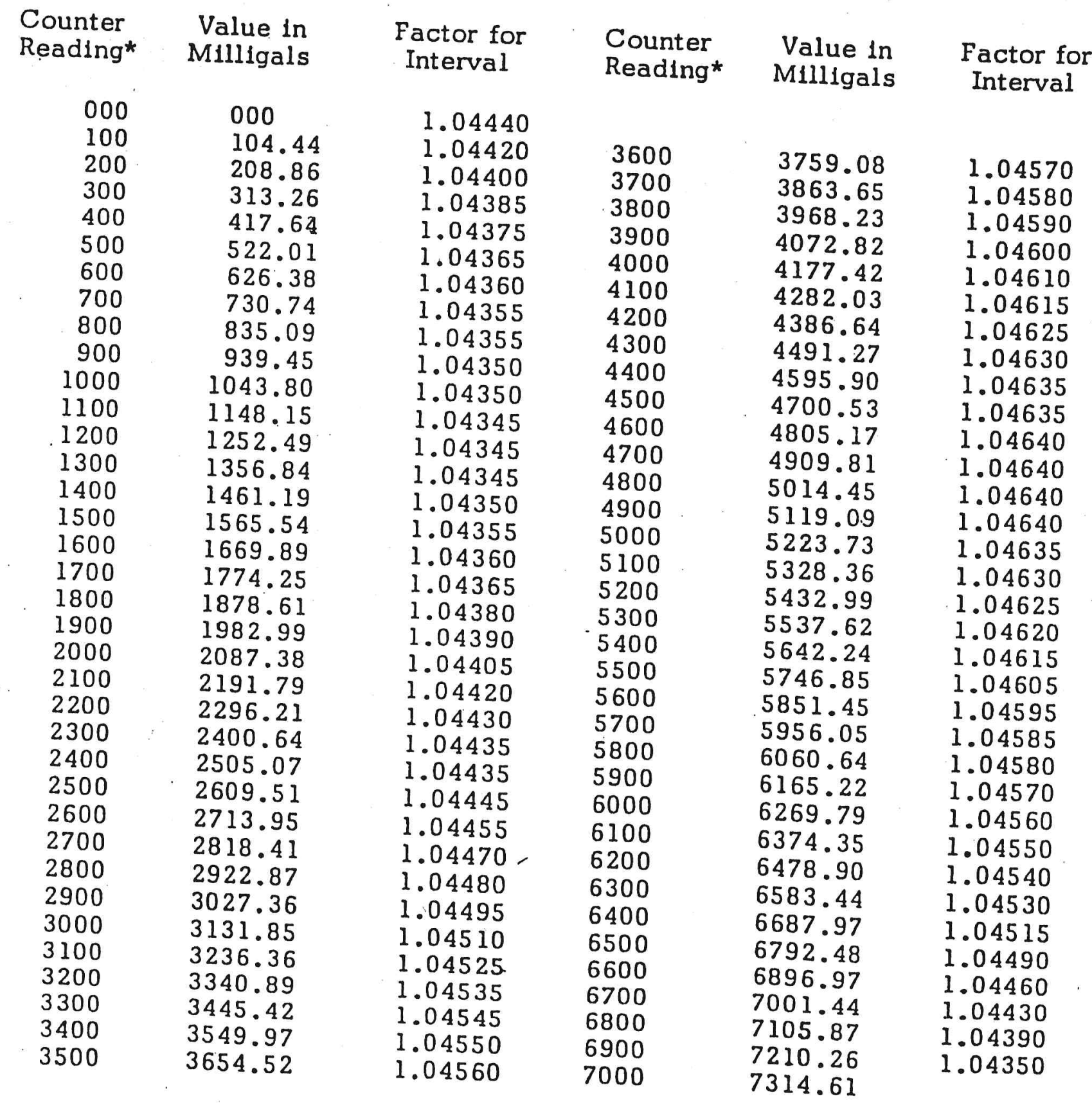

*NOTE: Right hand wheel on counter equals approximately .1 milligal.
AWS $7-18-62$ 


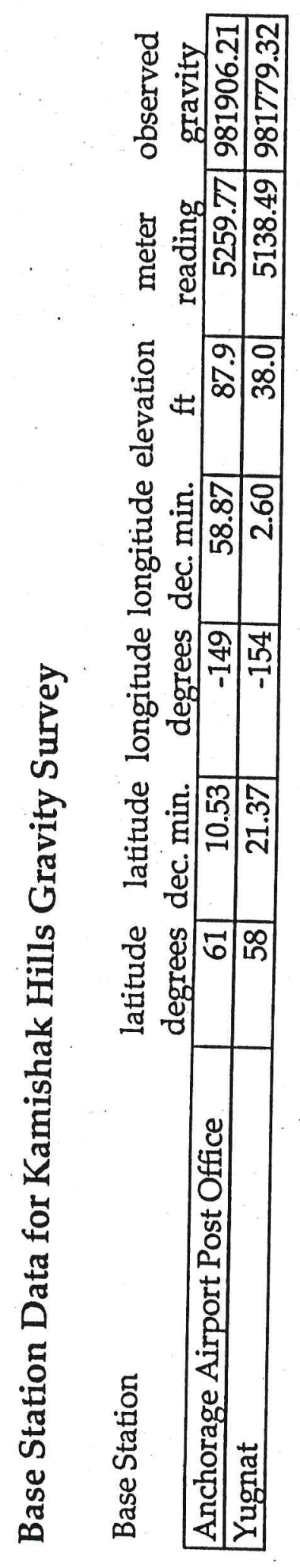


station year julian time latitude latitude longitude longitude elevation meter date hrs-min degrees dec. min. degrees dec.min. $\mathrm{ft}$ reading

\begin{tabular}{|c|c|c|c|c|c|c|c|c|c|}
\hline yugnat & 93 & 228 & 1047 & 58 & 21.37 & -154 & 2.60 & 38.0 & 5138.39 \\
\hline ia3-01 & 93 & 228 & 1134 & 59 & 3.35 & -153 & 54.58 & 814.0 & 5135.66 \\
\hline ia3-04 & 93 & 228 & 1149 & 59 & 2.76 & -154 & 1.34 & 1450.0 & 5098.74 \\
\hline ia3-05 & 93 & 228 & 1204 & 59 & 1.07 & -153 & 57.21 & 2012.0 & 5060.42 \\
\hline ia3-03 & 93 & 228 & 1219 & 59 & 0.65 & \begin{tabular}{|l|}
-153 \\
\end{tabular} & 55.38 & 1705.0 & 5078.93 \\
\hline ia3-02 & 93 & 228 & 1231 & 59 & 2.20 & -153 & 55.41 & 1585.0 & 5089.52 \\
\hline base 2 & 93 & 228 & 1249 & 59 & 3.87 & -153 & 49.41 & 5.0 & 5188.09 \\
\hline isle & 93 & 228 & 1349 & 59 & 6.52 & -153 & \begin{tabular}{l|}
52.58 \\
\end{tabular} & 0.0 & 5194.18 \\
\hline ia3-06 & 93 & 228 & 1404 & 59 & 0.20 & -153 & 52.68 & 1350.0 & 5100.35 \\
\hline ad6-01 & 93 & 228 & 1415 & 58 & 59.56 & -153 & 56.22 & 1570.0 & 5087.53 \\
\hline ad6-02 & 93 & 228 & 1424 & 58 & 58.25 & -153 & 56.14 & 1950.0 & 5062.15 \\
\hline ad6-03 & 93 & 228 & 1433 & 58 & 57.24 & -153 & 59.85 & 2665.0 & 5014.09 \\
\hline ad6-04 & 93 & 228 & 1442 & 58 & 55.48 & -153 & 59.08 & 2125.0 & 5045.11 \\
\hline ad6-05 & 93 & 228 & 1501 & 58 & 53.78 & -153 & 56.53 & 1205.0 & 5097.00 \\
\hline ad6-07 & 93 & 228 & 1511 & 58 & 53.98 & -153 & 52.65 & 1250.0 & 5099.57 \\
\hline yugnat & 93 & 228 & 1632 & 58 & 21.37 & -154 & 2.60 & 38.0 & 5138.45 \\
\hline yugnat & 93 & 229 & 947 & 58 & 21.37 & -154 & 2.60 & 38.0 & 5138.48 \\
\hline ad6-06 & 93 & 229 & 1017 & 58 & 52.65 & -153 & 59.92 & 2065.0 & 5043.64 \\
\hline ad6-11 & 93 & 229 & 1034 & 58 & 59.30 & -153 & 51.68 & 1230.0 & 5108.26 \\
\hline base2 & 93 & 229 & 1046 & 59 & 3.87 & -153 & 49.41 & 5.0 & 5188.09 \\
\hline ial-01 & 93 & 229 & 1051 & 59 & 6.26 & -153 & 43.96 & -10.0 & 5194.63 \\
\hline ia1-02 & 93 & 229 & 1104 & 59 & 5.85 & -153 & 43.54 & -5.0 & 5194.01 \\
\hline ia1-03 & 93 & 229 & 1114 & 59 & 5.09 & -153 & 42.20 & 5.0 & 5192.08 \\
\hline ia1-04 & 93 & 229 & 1122 & 59 & 4.26 & -153 & \begin{tabular}{|l|}
41.99 \\
\end{tabular} & 10.0 & 5189.28 \\
\hline ia1-05 & 93 & 229 & 1129 & 59 & 3.72 & -153 & 40.92 & 10.0 & 5188.71 \\
\hline ia1-06 & 93 & 229 & 1137 & 59 & 2.93 & -153 & 40.08 & 5.0 & 5187.32 \\
\hline ia 1-07 & 93 & 229 & 1149 & 59 & 2.24 & -153 & 39.44 & 5.0 & 5186.31 \\
\hline ia 1-08 & 93 & 229 & 1152 & 59 & 1.58 & -153 & 38.58 & 5.0 & 5185.80 \\
\hline ia1-09 & 93 & 229 & 1158 & 59 & 1.01 & -153 & 37.72 & 5.0 & 5184.54 \\
\hline ia1-10 & 93 & 229 & 1205 & 59 & 0.47 & -153 & 36.79 & 5.0 & 5184.12 \\
\hline ad5-04 & 93 & 229 & 1212 & 58 & 59.75 & -153 & 34.85 & 5.0 & 5185.20 \\
\hline ad5-05 & 93 & 229 & 1219 & 58 & 59.28 & -153 & 33.41 & 5.0 & 5186.92 \\
\hline $\operatorname{ad5} 5-06$ & 93 & 229 & 1226 & 58 & 58.58 & -153 & 32.29 & 10.0 & 5187.26 \\
\hline ad5-07 & 93 & 229 & 1238 & 58 & 58.32 & -153 & 30.16 & 140.0 & 5184.02 \\
\hline ad5-08 & 93 & 229 & 1243 & 58 & 57.31 & -153 & 31.74 & 1265.0 & 5112.66 \\
\hline ad5-09 & 93 & 229 & 1358 & 58 & 59.48 & -153 & 30.34 & 10.0 & 5191.91 \\
\hline ad5-01 & 93 & 229 & 1533 & 58 & 55.48 & -153 & 38.77 & 2165.0 & 5050.95 \\
\hline $\mathrm{d} 5-02$ & 93 & 229 & 1541 & 58 & 54.28 & -153 & 38.47 & 2470.0 & 5030.18 \\
\hline d5-03 & 93 & 229 & 1552 & 58 & 52.24 & -153 & 34.93 & 3620.0 & 4958.55 \\
\hline d6-14 & 93 & 229 & 1608 & 58 & 57.89 & -153 & 42.44 & 90.0 & 5173.72 \\
\hline d $6-13$ & 93 & 229 & 1618 & 58 & 58.37 & -153 & 44.83 & 405.0 & 5158.61 \\
\hline ad $6-12$ & 93 & 229 & 1628 & 58 & 58.61 & -153 & 48.28 & 650.0 & 5142.66 \\
\hline base2 & 93 & 229 & 1639 & 59 & 3.87 & -153 & 49.41 & 5.0 & 5188.05 \\
\hline
\end{tabular}


station year julian time latitude latitude longitude longitude elevation meter date hrs-min degrees dec. min. degrees dec.min. $\mathrm{ft}$ reading

\begin{tabular}{|c|c|r|r|r|r|r|r|r|r|}
\hline ia3-08 & 93 & 229 & 1652 & 59 & 1.78 & -154 & 2.71 & 1980.0 & 5062.87 \\
\hline ia3-09 & 93 & 229 & 1657 & 59 & 1.48 & -154 & 1.44 & 1815.0 & 5074.99 \\
\hline ia3-07 & 93 & 229 & 1704 & 59 & 0.65 & -154 & 0.68 & 2454.0 & 5031.56 \\
\hline kd1-01 & 93 & 229 & 1715 & 58 & 56.21 & -154 & 7.39 & 1220.0 & 5099.85 \\
\hline kd1-02 & 93 & 229 & 1722 & 58 & 55.74 & -154 & 4.75 & 2470.0 & 5018.24 \\
\hline kd1-03 & 93 & 229 & 1732 & 58 & 54.61 & -154 & 3.70 & 2915.0 & 4989.02 \\
\hline kd1-04 & 93 & 229 & 1738 & 58 & 54.87 & -154 & 5.46 & 2470.0 & 5018.47 \\
\hline kd1-05 & 93 & 229 & 1749 & 58 & 55.09 & -154 & 7.94 & 1220.0 & 5096.42 \\
\hline yugnat & 93 & 229 & 1827 & 58 & 21.37 & -154 & 2.60 & 38.0 & 5138.59 \\
\hline yugnat & 93 & 231 & 947 & 58 & 21.37 & -154 & 2.60 & 38.0 & 5138.59 \\
\hline ia1-11 & 93 & 231 & 1033 & 59 & 4.89 & -153 & 43.64 & -10.0 & 5191.89 \\
\hline ia1-12 & 93 & 231 & 1039 & 59 & 4.46 & -153 & 44.75 & -10.0 & 5190.89 \\
\hline ia3-10 & 93 & 231 & 1044 & 59 & 4.65 & -153 & 47.33 & -10.0 & 5191.77 \\
\hline ia3-11 & 93 & 231 & 1051 & 59 & 4.47 & -153 & 48.56 & -10.0 & 5191.57 \\
\hline base2 & 93 & 231 & 1057 & 59 & 3.87 & -153 & 49.41 & 5.0 & 5188.21 \\
\hline ia3-12 & 93 & 231 & 1102 & 59 & 3.27 & -153 & 51.50 & -5.0 & 5187.03 \\
\hline ia3-13 & 93 & 231 & 1108 & 59 & 3.61 & -153 & 52.91 & -5.0 & 5187.74 \\
\hline ia3-14 & 93 & 231 & 1114 & 59 & 3.63 & -153 & 54.49 & -5.0 & 5186.42 \\
\hline ia3-15 & 93 & 231 & 1119 & 59 & 3.71 & -153 & 55.52 & 0.0 & 5187.84 \\
\hline ia3-16 & 93 & 231 & 1129 & 59 & 4.07 & -153 & 56.86 & 0.0 & 5186.47 \\
\hline ia3-17a & 93 & 231 & 1134 & 59 & 4.16 & -153 & 57.63 & 0.0 & 5185.45 \\
\hline ia3-17b & 93 & 231 & 1139 & 59 & 4.22 & -153 & 58.57 & 0.0 & 5186.39 \\
\hline ia3-18 & 93 & 231 & 1144 & 59 & 4.35 & -153 & 59.75 & 0.0 & 5186.39 \\
\hline ia3-19 & 93 & 231 & 1149 & 59 & 4.52 & -154 & 0.94 & 5.0 & 5189.60 \\
\hline ia3-20 & 93 & 231 & 1154 & 59 & 4.52 & -154 & 2.08 & -5.0 & 5189.25 \\
\hline ia3-21 & 93 & 231 & 1159 & 59 & 4.43 & -154 & 3.86 & -10.0 & 5190.25 \\
\hline base2 & 93 & 231 & 1214 & 59 & 3.87 & -153 & 49.41 & 5.0 & 5188.15 \\
\hline ad6-11 & 93 & 231 & 1232 & 58 & 53.73 & -153 & 48.55 & 495.0 & 5144.04 \\
\hline ad6-15 & 93 & 231 & 1239 & 58 & 54.50 & -153 & 43.07 & 815.0 & 5126.88 \\
\hline yugnat & 93 & 231 & 1515 & 58 & 21.37 & -154 & 2.60 & 38.0 & 5138.49 \\
\hline & & & & & & & & & \\
\hline
\end{tabular}




\begin{tabular}{|c|c|c|c|c|c|c|c|}
\hline SECTION NAME & CODE & TRS & QUAD & DATE & MEASUREDBY & INSTRUMENT & \\
\hline DOUGLAS R. IS. & 93KAT 106 & N/SW 2-13S-2 & LLIAMNA A1/A & $8 / 20 / 93$ & ntered on comp & SCINTEX GIS-5; Background =463@ 0 Feet & \\
\hline & & & & & & & \\
\hline & & & & & & & \\
\hline DEPTH & GR1 & GR2 & GR3 & GR AVE. & LITHOLOGY & COMMENTS & \\
\hline 0 & 483 & 507 & 530 & 507 & cgr ss & & \\
\hline 5 & 501 & 505 & 511 & 506 & cgr ss & & \\
\hline 10 & 504 & 465 & 516 & 495 & cgr ss & & \\
\hline 15 & 507 & 502 & 495 & 501 & cgr ss & & \\
\hline 20 & 548 & 550 & 551 & 550 & cgr ss & & \\
\hline 25 & 592 & 577 & 547 & 572 & cgr ss & & \\
\hline 30 & 636 & 661 & 654 & 650 & mgr ss & & \\
\hline 35 & 647 & 643 & 649 & 646 & mgr ss & & \\
\hline 40 & 662 & 667 & 664 & 664 & mgr ss & & \\
\hline 45 & 585 & 589 & 604 & 593 & mgr ss & & \\
\hline 50 & 715 & 796 & 617 & 709 & fgr ss & & \\
\hline 55 & 567 & 600 & 601 & 589 & fgr ss & & \\
\hline 60 & 466 & 497 & 509 & 491 & fgr ss & & \\
\hline 65 & 499 & 529 & 549 & 526 & fgr ss & & \\
\hline 70 & 491 & 447 & 479 & 472 & cgr ss & & \\
\hline 75 & 502 & 499 & 527 & 509 & cgr ss & & \\
\hline 80 & 515 & 495 & 532 & 514 & cgr ss & & \\
\hline 85 & 562 & 533 & 520 & 538 & cgr ss & & \\
\hline 90 & 488 & 555 & 537 & 527 & cgr ss & & \\
\hline 95 & 560 & 534 & 551 & 548 & cgr ss & & \\
\hline 100 & 563 & 533 & 530 & 542 & cgr ss & & \\
\hline 105 & 528 & 561 & 526 & 538 & cgr ss & & \\
\hline 110 & 569 & 530 & 577 & 559 & cgr ss & & \\
\hline 115 & 597 & 538 & 572 & 569 & cgr ss & & \\
\hline 120 & 595 & 564 & 538 & 566 & cgr ss & & \\
\hline 125 & 650 & 670 & 627 & 649 & cgr ss & & \\
\hline 130 & 572 & 584 & 559 & 572 & mgr ss & & \\
\hline
\end{tabular}




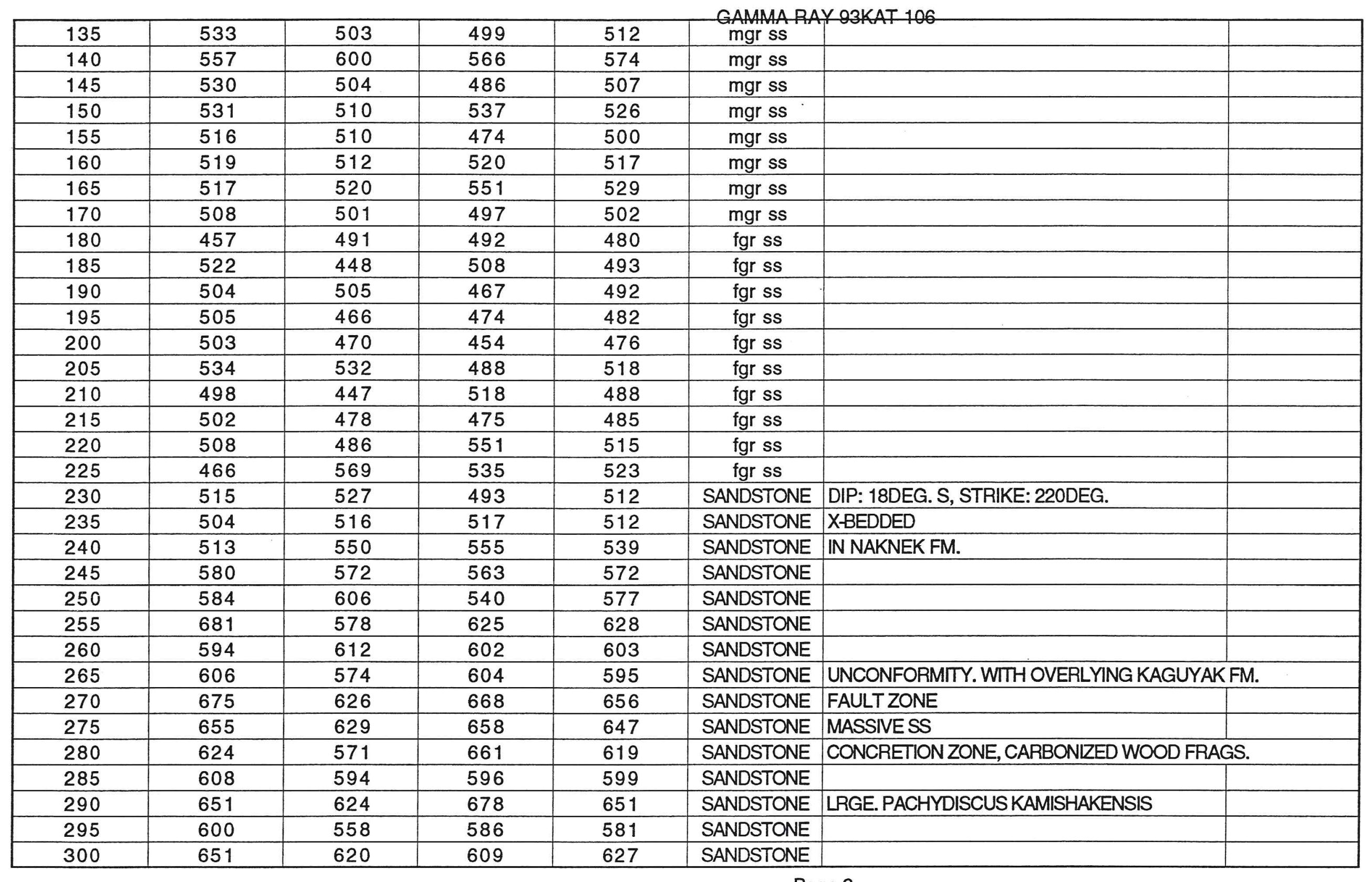




\begin{tabular}{|c|c|c|c|c|c|c|c|}
\hline 305 & 600 & 602 & 575 & 592 & SANDSTONE & & \\
\hline 310 & 593 & 652 & 610 & 618 & SANDSTONE & & \\
\hline 315 & 651 & 584 & 614 & 616 & SANDSTONE & 2 SPECIES AMMONTES & \\
\hline 320 & 632 & 621 & 617 & 623 & SANDSTONE & & \\
\hline 325 & 615 & 567 & 613 & 598 & SANDSTONE & & \\
\hline 330 & 615 & 586 & 589 & 597 & SANDSTONE & SAND COARSENS UPWARD F-M GR. & \\
\hline 335 & 519 & 612 & 577 & 569 & SANDSTONE & & \\
\hline 340 & 630 & 577 & 598 & 602 & SANDSTONE & PACHYDISCUS KAMISHAKENSIS & \\
\hline \multirow[t]{25}{*}{345} & 640 & 614 & 596 & 617 & SANDSTONE & FAULT ZONE @ END OF SEC. & \\
\hline & & & & 0 & & END BG GR: 466. & \\
\hline & & & & 0 & & 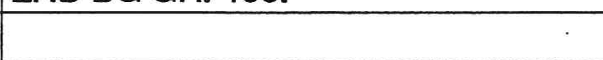 & \\
\hline & & & & 0 & & & \\
\hline & & & & 0 & & & \\
\hline & & & & 0 & & & \\
\hline & & & & 0 & & & \\
\hline & & & & 0 & & & \\
\hline & & & & 0 & & & \\
\hline & & & & 0 & & & \\
\hline & & & & 0 & & & \\
\hline & & & & 0 & & & \\
\hline & & & & 0 & & & \\
\hline & & & & 0 & & & \\
\hline & & & & 0 & & & \\
\hline & & & & 0 & & & \\
\hline & & & & 0 & & & \\
\hline & & & & 0 & & & \\
\hline & & & & 0 & & & \\
\hline & & & & 0 & & & \\
\hline & & & & 0 & & & \\
\hline & & & & 0 & & & \\
\hline & & & & 0 & & & \\
\hline & & & & 0 & & & \\
\hline & & & & 0 & & & \\
\hline
\end{tabular}




\begin{tabular}{|c|c|c|c|c|c|c|c|c|c|c|c|c|c|c|}
\hline & & & & & & & & Paleocurrent-93 fld & & & & & & \\
\hline & & & & & & & & & & & & & & \\
\hline & & & & & & & & Attitude; major & Depth & & Paleocurrent & & If avg. & \\
\hline Location & Stratigraphic & Geographic & Sec. & Twnshp & Rng & Quad & Depositional & bedding & in Meas. & Type of current & direction & Quality & \# of meas. & Average \\
\hline & unit & Area & & & & & facies & (Dip / Dip dir.) & Section & indicator & $(\mathrm{B},=$ Bidirec. $)$ & & $(n>1)$ & \begin{tabular}{|l} 
paleocurrent \\
\end{tabular} \\
\hline 93KAT1 & Kk & Mt. Pedmar & 24 & $25 \mathrm{~S}$ & $34 \mathrm{~W}$ & Mt. Katmai A3 & & & $50^{\prime}$ in & trough $\mathrm{xbdd}$ & $164^{\circ}$ & moderate & & \\
\hline 93KAT1 & Kk & Mt. Pedmar & 24 & $25 \mathrm{~S}$ & $34 \mathrm{~W}$ & Mt. Katmai A3 & & & meas. sec. & trough $x$ bdd & $160^{\circ}$ & moderate & & \\
\hline 93KAT1 & $\mathrm{Kk}$ & Mt. Pedmar & 24 & $25 \mathrm{~S}$ & $34 \mathrm{~W}$ & Mt. Katmai A3 & & & 93KAT105 & trough xbdd & $230^{\circ}$ & moderate & & \\
\hline 93KAT101 & Khl & Kamishak Mountain & 23 & $15 \mathrm{~S}$ & $29 \mathrm{~W}$ & Mt. Katmai D1 & & & $55^{\prime}$ & flame & $85^{\circ}$ & - & & \\
\hline 93KAT103 & $\mathrm{Jn}, \mathrm{Khl}$ & Twin Glaciers & 12 & $15 \mathrm{~S}$ & $27 \mathrm{~W}$ & Afognak D6 & & $9 / 172$ & $58^{1}$ & trough $x$ bdd & $300^{\circ}$ & & 3 & 293 \\
\hline 93KAT103 & $\mathrm{Jn}, \mathrm{Khl}$ & Twin Glaciers & 12 & 155 & $27 \mathrm{~W}$ & Afognak D6 & & & $58^{\prime}$ & trough $\times$ bdd & $285^{\circ}$ & & & \\
\hline 93KAT 103 & $\mathrm{Jn}, \mathrm{Khl}$ & Twin Glaciers & 12 & $15 \mathrm{~S}$ & $27 \mathrm{~W}$ & Afognak D6 & & & $58^{1}$ & trough $x b d d$ & $295^{\circ}$ & & & \\
\hline 93KAT105 & $\mathrm{Kp}, \mathrm{Kk}$ & Mt. Pedmar & 24 & $25 \mathrm{~S}$ & $34 \mathrm{~W}$ & Mt. Katmai A3 & & $12 / 35$ at $30^{\prime}$ & $246^{\prime}$ & ripples & $194^{\circ}$ & & & \\
\hline 93KAT105 & $\mathrm{Kp}, \mathrm{Kk}$ & Mt. Pedmar & 24 & $25 \mathrm{~S}$ & $34 \mathrm{~W}$ & Mt. Katmai A3 & & $14 / 119$ at $85^{\prime}$ & $350^{\prime}$ & trough $x$ bdd & $175^{\circ}$ & & 4 & 191 \\
\hline 93KAT105 & $\mathrm{Kp}, \mathrm{Kk}$ & Mt. Pedmar & 24 & $25 \mathrm{~S}$ & $34 \mathrm{~W}$ & Mt. Katmai A3 & & $8 / 131$ at $160^{\circ}$ & $362^{1}$ & trough $x$ bdd & $150^{\circ}$ & & & \\
\hline 93KAT105 & $\mathrm{Kp}, \mathrm{Kk}$ & Mt. Pedmar & 24 & $25 \mathrm{~S}$ & $34 \mathrm{~W}$ & Mt. Katmai $\mathrm{A} 3$ & & $11 / 149$ at $175^{\prime}$ & $362^{\prime}$ & trough $\times b d d$ & $320^{\circ}$ & & & \\
\hline 93KAT105 & $\mathrm{Kp}, \mathrm{Kk}$ & Mt. Pedmar & 24 & $25 \mathrm{~S}$ & $34 \mathrm{~W}$ & Mt. Katmai A3 & & & $366^{\prime}$ & trough $x \mathrm{bdd}$ & $120^{\circ}$ & & & \\
\hline 93KAT106 & Jn & Douglas River Island & 2 & 135 & $27 \mathrm{~W}$ & lliamna A1\&2 & & $8 / 155$ & $8^{\prime}$ & trough $\mathrm{xbdd}$ & $110^{\circ}$ & & & \\
\hline 93KAT106 & In & Douglas River Island & 2 & $13 \mathrm{~S}$ & $27 \mathrm{~W}$ & lliamna A1\&2 & & & $11^{\prime}$ & ripple & $250^{\circ}$ & & 3 & 208 \\
\hline 93KAT106 & $\mathrm{Jn}$ & Douglas River Island & 2 & 135 & $27 \mathrm{~W}$ & Iliamna A1\&2 & & & $11^{\prime}$ & ripple & $269^{\circ}$ & & & \\
\hline 93KAT106 & Jn & Douglas River Island & 2 & 135 & $27 \mathrm{~W}$ & illiamna A1\&2 & & & $11^{\prime}$ & ripple & $105^{\circ}$ & & & \\
\hline 93KAT106 & Jn & Douglas River Island & 2 & 135 & $27 \mathrm{~W}$ & lliamna A1\&2 & & & $12^{\prime}$ & trough $\times$ bdd & $140^{\circ}$ & & 16 & 230 \\
\hline 93KAT106 & $\mathrm{Jn}$ & Douglas River Island & 2 & 135 & $27 \mathrm{~W}$ & lliamna A1\&2. & & & $16^{\prime}$ & trough $x b d d$ & $305^{\circ}$ & & & \\
\hline 93KAT1106 & $\mathrm{Jn}$ & \begin{tabular}{|l|} 
Douglas River Island \\
\end{tabular} & 2 & 135 & $27 \mathrm{~W}$ & Iliamna A1\&2 & & & $17^{\prime}$ & trough $\mathrm{xbdd}$ & $296^{\circ}$ & & & \\
\hline 93KAT106 & Jn & Douglas River Island & 2 & $13 S$ & $27 \mathrm{~W}$ & lliamna A182 & & & $35^{\prime}$ & trough $x$ bdd & $260^{\circ}$ & & & \\
\hline 93KAT106 & $J n$ & Douglas River Island & 2 & 135 & $27 \mathrm{~W}$ & Iliamna A1\&2 & & & $37^{\prime}$ & trough $x b d d$ & $255^{\circ}$ & & & \\
\hline 93KAT106 & Jn & Douglas River Island & 2 & $13 S$ & $27 \mathrm{~W}$ & lliamna A1\&2 & & & $37^{\prime}$ & trough $x \mathrm{bdd}$ & $200^{\circ}$ & & & \\
\hline 93КАТ 106 & Jn & Douglas River Island & 2 & $13 \mathrm{~S}$ & $27 \mathrm{~W}$ & lliamna A1\&2 & & & $37^{\prime}$ & trough $x \mathrm{bdd}$ & $245^{\circ}$ & & & \\
\hline 93KAT106 & Jn & Douglas River Island & 2 & $13 S$ & $27 \mathrm{~W}$ & lliamna A1\&2 & & & $38^{\prime}$ & trough $\times$ bdd & $265^{\circ}$ & & & \\
\hline 93KAT106 & Jn & Douglas River Island & 2 & $13 \mathrm{~S}$ & $27 W$ & lliamna A1\&2 & & & $41^{\prime}$ & trough $\mathrm{xbdd}$ & $190^{\circ}$ & & & \\
\hline 93KAT106 & In & Douglas River Island & 2 & $13 \mathrm{~S}$ & $27 \mathrm{~W}$ & Iliamna A1\&2 & & & $43^{\prime}$ & trough $x \mathrm{bdd}$ & $220^{\circ}$ & & & \\
\hline 93KAT106 & Jn & Douglas River Island & 2 & $13 \mathrm{~S}$ & $27 \mathrm{~W}$ & Iliamna A1\&2 & & & $46^{\prime}$ & trough $x b d d$ & $340^{\circ}$ & & & \\
\hline 93KAT106 & Jn & Douglas River Island & 2 & $13 S$ & $27 \mathrm{~W}$ & lliamna A1\&2 & & & $46^{\prime}$ & trough $x b d d$ & $330^{\circ}$ & & & \\
\hline 93KAT106 & Jn & Douglas River Island & 2 & $13 \mathrm{~S}$ & $27 \mathrm{~W}$ & lliamna A1\&2 & & & $97^{\prime}$ & trough $x \mathrm{bdd}$ & $175^{\circ}$ & & & \\
\hline 93KAT106 & In & Douglas River Island & 2 & $13 S$ & $27 \mathrm{~W}$ & liamna A1\&2 & & & $99^{\prime}$ & trough $x \mathrm{bdd}$ & $195^{\circ}$ & & & \\
\hline 93KAT106 & Jn & Douglas River Island & 2 & $13 \mathrm{~S}$ & $27 \mathrm{~W}$ & liamna A182 & & & $115^{\prime}$ & trough $x \mathrm{bdd}$ & $10^{\circ}$ & & & \\
\hline 93KAT106 & Jn & Douglas River Island & 2 & $13 \mathrm{~S}$ & $27 \mathrm{~W}$ & liamna A1\&2 & & & $117^{\prime}$ & trough $x \mathrm{bdd}$ & $260^{\circ}$ & & & \\
\hline 93KAT106 & $\mathrm{Jn}$ & \begin{tabular}{|l|} 
Douglas River Island \\
\end{tabular} & 2 & $13 \mathrm{~S}$ & $27 \mathrm{~W}$ & lliamna A182 & & $17 / 159$ & $226^{\prime}$ & trough $\times$ bdd & $95^{\circ}$ & & 13 & 133 \\
\hline 93KAT106 & Ks & Douglas River Island & 2 & $13 \mathrm{~S}$ & $27 \mathrm{~W}$ & Iliamna A1\&2 & & $18 / 130$ & $245^{\prime}$ & tabular xbdd & $270^{\circ}$ & & & \\
\hline 93KAT106 & Ks & Douglas River Island & 2 & $13 S$ & $27 \mathrm{~W}$ & lliamna $A 1 \& 2$ & & & $245^{\prime}$ & tabular xbdd & $145^{\circ}$ & & & \\
\hline 93KAT106 & Ks & Douglas River Island & 2 & 135 & $27 \mathrm{~W}$ & Uliamna A1\&2 & & & $245^{\prime}$ & tabular xbdd & $90^{\circ}$ & & & \\
\hline 93KAT106 & Ks & Douglas River Island & 2 & 135 & $27 \mathrm{~W}$ & lliamna A1\&2 & & & $245^{\prime}$ & tabular $x$ bdd & $240^{\circ}$ & & & \\
\hline 93КАТT106 & Ks & Douglas River Island & 2 & 135 & $27 \mathrm{~W}$ & Iliamna A1\&2 & & & $245^{\prime}$ & tabular xbdd & $165^{\circ}$ & & & \\
\hline 93KAT106 & Ks & Douglas River Island & 2 & 135 & $27 \mathrm{~W}$ & lliamna A182 & & & $245^{\prime}$ & tabular xbdd & $130^{\circ}$ & & & \\
\hline 93KAT106 & Ks & Douglas River Island & 2 & $13 S$ & $27 \mathrm{~W}$ & lliamna A182 & & & $258^{\prime}$ & tabular $\mathrm{xbdd}$ & $180^{\circ}$ & & & \\
\hline
\end{tabular}




\begin{tabular}{|c|c|c|c|c|c|c|c|c|c|c|c|c|c|}
\hline 93KAT106 & Ks & Douglas River Island & 2 & 135 & $27 \mathrm{~W}$ & Lliamna A1\&2 & & Paleocurrent-93 fld prg 261' & tabular xbdd & $28^{\circ}$ & & & \\
\hline 93KAT106 & Ks & Douglas River Island & 2 & 135 & $27 \mathrm{~W}$ & lliamna A1\&2 & & $261^{\prime}$ & tabular xbdd & $29^{\circ}$ & & & \\
\hline 93KAT106 & Ks & Douglas River Island & 2 & $13 S$ & $27 \mathrm{~W}$ & lliamna A1\&2 & & $261^{\prime}$ & tabular xbdd & $30^{\circ}$ & & & \\
\hline 93KAT106 & Ks & Douglas River Island & 2 & 135 & $27 \mathrm{~W}$ & lliamna A182 & w/in the & $267^{\prime}$ & tabular xbdd & $170^{\circ}$ & & & \\
\hline 93KAT106 & Ks & Douglas River Island & 2 & $13 S$ & $27 \mathrm{~W}$ & lliamna A1\&2 & channels & $267^{\prime}$ & tabular xbdd & $155^{\circ}$ & & & \\
\hline 93KAT107 & $\mathrm{Jn} / \mathrm{Ks}$ & Douglas River & 10 & $13 S$ & $27 \mathrm{~W}$ & lliamna A3 & channel margins & & channel margin & $150^{\circ}$ & fair & 10 & 131 \\
\hline 93KAT107 & $\mathrm{Jn} / \mathrm{Ks}$ & Douglas River & 10 & $13 S$ & $27 \mathrm{~W}$ & lilamna A3 & in the $\mathrm{Jn}$ and & & channel margin & $137^{\circ}$ & poor & & \\
\hline 93КAT107 & $\mathrm{Jn} / \mathrm{Ks}$ & Douglas River & 10 & 135 & $27 \mathrm{~W}$ & lliamna A3 & filled with $K s$ & & channel margin & $106^{\circ}$ & good & & \\
\hline 93KAT107 & $\mathrm{Jn} / \mathrm{Ks}$ & Douglas River & 10 & $13 \mathrm{~S}$ & $27 \mathrm{~W}$ & lliamna $\mathbf{A} 3$ & and directions & & channel margin & $123^{\circ}$ & good & & \\
\hline 93KAT107 & $\mathrm{Jn} / \mathrm{Ks}$ & Douglas River & 10 & 135 & $27 \mathrm{~W}$ & lliamna A3 & are bidirec. & & channel margin & $137^{\circ}$ & fair & & \\
\hline 93KAT107 & $\mathrm{Jn} / \mathrm{Ks}$ & Douglas River & 10 & $13 S$ & $27 \mathrm{~W}$ & liliamna $\mathrm{A} 3$ & & & channel margin & $134^{\circ}$ & poor & & \\
\hline 93KAT107 & $\mathrm{Jn} / \mathrm{Ks}$ & Douglas River & 10 & 135 & $27 \mathrm{~W}$ & Iliamna A3 & & & channel margin & $149^{\circ}$ & poor & & \\
\hline 93КАТ107 & $\mathrm{Jn} / \mathrm{Ks}$ & Douglas River & 10 & $13 S$ & $27 \mathrm{~W}$ & Iliamna A3 & & & channel margin & $122^{\circ}$ & good & & \\
\hline 93KAT107 & $\mathrm{Jn} / \mathrm{Ks}$ & Douglas River & 10 & 135 & $27 W$ & lliamna $\mathrm{A} 3$ & & & channel margin & $109^{\circ}$ & fair & & \\
\hline 93KAT107 & $\mathrm{Jn} / \mathrm{Ks}$ & Douglas River & 10 & 135 & $27 \mathrm{~W}$ & lliamna $\mathrm{A} 3$ & & & channel margin & $147^{\circ}$ & good & & \\
\hline 93КAT107 & Ks & Douglas River & 10 & $13 S$ & $27 \mathrm{~W}$ & lliamna $\mathrm{A} 3$ & & & pebble imbrication & $165^{\circ}$ & & 7 & 149 \\
\hline 93КАТ 107 & Ks & Douglas River & 10 & $13 S$ & $27 \mathrm{~W}$ & Iliamna $\mathrm{A} 3$ & & & pebble imbrication & $158^{\circ}$ & & & \\
\hline 93КАT 107 & Ks & Douglas River & 10 & 135 & $27 \mathrm{~W}$ & lliamna $A 3$ & & & pebble imbrication & $155^{\circ}$ & & & \\
\hline 93КАТ 107 & Ks & \begin{tabular}{|l|l|} 
Douglas River \\
\end{tabular} & 10 & $13 S$ & $27 W$ & lliamna $\mathrm{A} 3$ & & & pebble imbrication & $56^{\circ}$ & & & \\
\hline 93КАT107 & Ks & Douglas River & 10 & $13 S$ & $27 \mathrm{~W}$ & lliamna $\mathrm{A} 3$ & & & pebble imbrication & $154^{\circ}$ & & & \\
\hline 93KAT107 & Ks & \begin{tabular}{|l} 
Douglas River \\
\end{tabular} & 10 & 135 & $27 W$ & lliamna $\mathrm{A} 3$ & & & pebble imbrication & $175^{\circ}$ & & & \\
\hline 93КAT 107 & Ks & Douglas River & 10 & $13 S$ & $27 \mathrm{~W}$ & lliamna $\mathrm{A} 3$ & & & pebble imbrication & $181^{\circ}$ & & & \\
\hline 93KAT107 & Ks & Douglas River & 10 & 135 & $27 \mathrm{~W}$ & lliamna A3 & & & belemn. lineations & $250^{\circ}$ & & 16 & 158 \\
\hline 93KAT107 & Ks & Douglas River & 10 & 135 & $27 \mathrm{~W}$ & lliamna A3 & & & belemn. lineations & $230^{\circ}$ & & & \\
\hline 93KAT107 & Ks & \begin{tabular}{|l} 
Douglas River \\
\end{tabular} & 10 & $13 S$ & $27 \mathrm{~W}$ & lliamna $A 3$ & & & belemn. lineations & $232^{\circ}$ & & & \\
\hline 93КАТ 107 & Ks & \begin{tabular}{|l} 
Douglas River \\
\end{tabular} & 10 & 135 & $27 \mathrm{~W}$ & lliamna $A 3$ & & & belemn. lineations & $50^{\circ}$ & & & \\
\hline 93KAT107 & Ks & \begin{tabular}{|l} 
Douglas River \\
\end{tabular} & 10 & 135 & $27 \mathrm{~W}$ & lliamna A3 & & & belemn. lineations & $68^{\circ}$ & measurm & & \\
\hline 93KAT107 & Ks & Douglas River & 10 & $13 S$ & $27 \mathrm{~W}$ & lliamna $\mathrm{A} 3$ & & & belemn. lineations & $250^{\circ}$ & in dir. of & & \\
\hline 93KAT107 & Ks & Douglas River & 10 & $13 S$ & $27 \mathrm{~W}$ & lliamna $A 3$ & & & belemn. lineations & $237^{\circ}$ & pointed $e$ & & \\
\hline 93КAT107 & Ks & Douglas River & 10 & $13 S$ & $27 \mathrm{~W}$ & lliamna A3 & & & belemn. lineations & $239^{\circ}$ & of belem. & & \\
\hline 93KAT107 & Ks & Douglas River & 10 & $13 S$ & $27 \mathrm{~W}$ & lliamna $\mathrm{A} 3$ & & & belemn. lineations & $69^{\circ}$ & & & \\
\hline 93KAT107 & Ks & Douglas River & 10 & $13 S$ & $27 \mathrm{~W}$ & lliamna $\mathrm{A} 3$ & & & belemn. lineations & $26^{\circ}$ & & & \\
\hline 93КАТ107 & Ks & Douglas River & 10 & 135 & $27 \mathrm{~W}$ & Iliamna $\mathrm{A3}$ & & & belemn. lineations & $74^{\circ}$ & & & \\
\hline 93KAT107 & Ks & Douglas River & 10 & $13 \mathrm{~S}$ & $27 \mathrm{~W}$ & lliamna $\mathrm{A} 3$ & & & belemn. lineations & $242^{\circ}$ & & & \\
\hline 93KAT107 & Ks & Douglas River & 10 & $13 \mathrm{~S}$ & $27 \mathrm{~W}$ & lliamna A3 & & & beiemn. lineations & $262^{\circ}$ & & & \\
\hline 93KAT107 & Ks & Douglas River & 10 & $13 S$ & $27 \mathrm{~W}$ & lliamna A3 & & & belemn. lineations & $71^{\circ}$ & & & \\
\hline 93KAT107 & Ks & Douglas River & 10 & $13 \mathrm{~S}$ & $27 \mathrm{~W}$ & lliamna A3 & & & belemn. lineations & $109^{\circ}$ & & & \\
\hline 93КAT107 & Ks & Douglas River & 10 & 135 & $27 \mathrm{~W}$ & lliamna $\mathrm{A} 3$ & & & belemn. lineations & $113^{\circ}$ & & & \\
\hline 93KAT110 & Ks & N Kamishak hills & 31 & $13 S$ & $28 \mathrm{~W}$ & lliamna A3 & & $94^{\prime}$ & ripple & $155^{\circ}$ & poor & & \\
\hline 93КАT3 & Kk & Mt. Pedmar & 17 & 255 & $33 \mathrm{~W}$ & Mt. Katmai A3 & & & ripple & $156^{\circ}$ & poor & & \\
\hline 93КАТЗ & Kk & Mt. Pedmar & 17 & $25 S$ & $33 \mathrm{~W}$ & Mt. Katmai A3 & & & ripple & $180^{\circ}$ & poor & & \\
\hline 93КАТЗ & $\mathrm{Kk}$ & Mt. Pedmar & 17 & $25 \mathrm{~S}$ & $33 \mathrm{~W}$ & Mt. Katmai $\mathrm{A} 3$ & & & ripple $(T c)$ & $90^{\circ}$ & fair & & \\
\hline 93КАТ6C & $\mathrm{Kk}$ & Mt. Pedmar & 17 & $25 \mathrm{~S}$ & $33 \mathrm{~W}$ & Mt. Katmai A3 & & near base & Tcd,Tbc,Tc & $105^{\circ}$ & good & 9 & 103 \\
\hline 93КАT6C & Kk & Mt. Pedmar & 17 & $25 \mathrm{~S}$ & $33 \mathrm{~W}$ & Mt. Katmai A3 & & of cong. & Tcd,Tbc,Tc & $100^{\circ}$ & good & & \\
\hline 93KAT6C & $\mathrm{kk}$ & Mt. Pedmar & 17 & $25 \mathrm{~S}$ & $33 W$ & Mt. Katmai A3 & & at $430^{\circ}$ in & Tcd,Tbc,Tc & $116^{\circ}$ & good & & \\
\hline
\end{tabular}

Page 2 


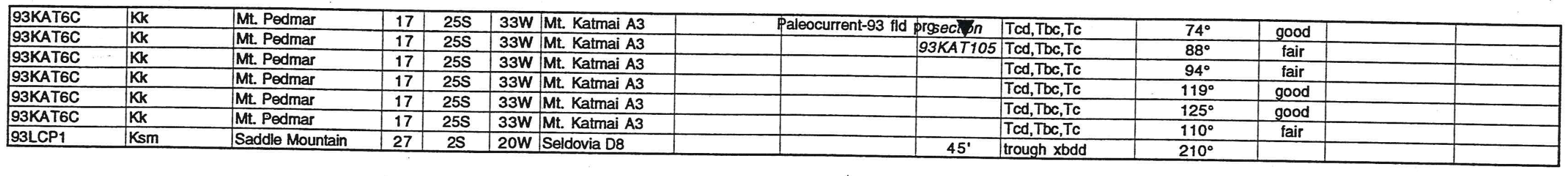




\begin{tabular}{|c|c|c|}
\hline \multicolumn{2}{|c|}{ 93KAT107 CLAST TYPE COUNT } & \\
\hline \multicolumn{3}{|l|}{ 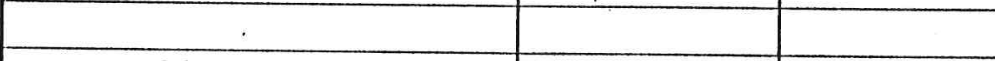 } \\
\hline LITHOLOGY & TOTAL & PERCENT \\
\hline Porphyritic Dacite & 10 & $10 \%$ \\
\hline Aphanitic Volcanic & 5 & $5 \%$ \\
\hline Micro-diorite & 6 & $6 \%$ \\
\hline Green Silicic Tuff & 28 & $28 \%$ \\
\hline Silicic Volcanic & 3 & $3 \%$ \\
\hline White Quartz & 15 & $15 \%$ \\
\hline Porphyritic Andesite & 19 & $19 \%$ \\
\hline Red Silicic Volcanic & 5 & $5 \%$ \\
\hline Silicic Volcanic & 6 & $6 \%$ \\
\hline Epidote & 1 & $1 \%$ \\
\hline Volcanic Sandstone & 1 & $1 \%$ \\
\hline Dark Grey Chert & 1 & $1 \%$ \\
\hline Total Count & 100 & \\
\hline
\end{tabular}

Page 1 


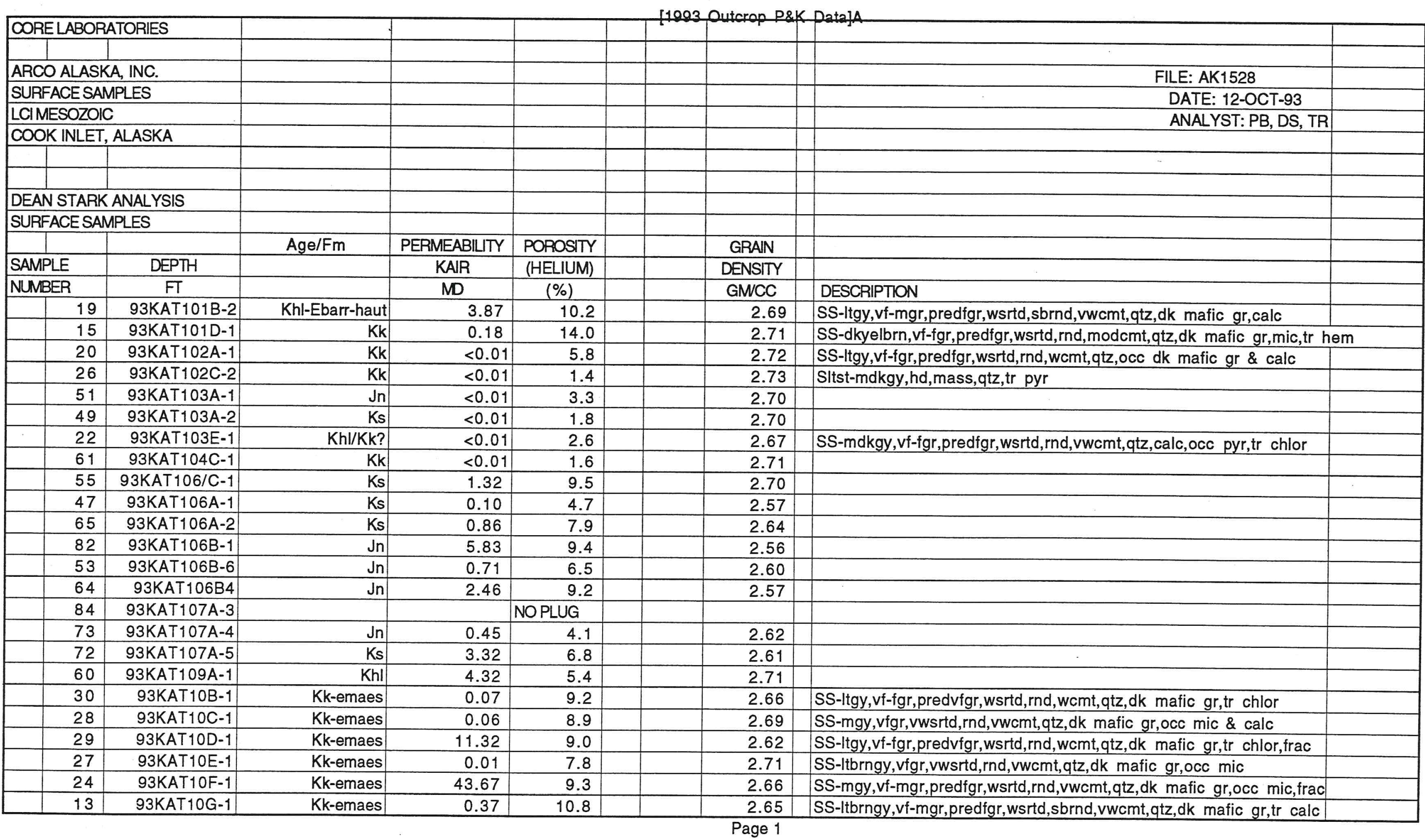




\begin{tabular}{|c|c|c|c|c|c|c|c|c|}
\hline 33 & 93KAT10I-1 & $\mathrm{Kk}$ & 0.05 & 5.9 & & 2.71 & SS-mdkgy,vf-fgr,predvfgr,wsrtd,rnd,vwcmt,qtz,dk mafic gr,tr calc & \\
\hline 31 & 93KAT10J-1 & $\mathrm{Kk}$ & 0.02 & 9.4 & & 2.71 & SS-mdkgy,vfgr,vwsitd,rnd,vwcmt,qtz,dk mafic gr,occ mic \& calc & \\
\hline 32 & 93KAT10L-1 & Khl-ebarr & 1.41 & 18.9 & & 2.67 & SS-ltgy,vf-fgr,predvfgr,wsrtd,rnd,wcmt,qtz,calc,dk mafic gr,occ chlor & \\
\hline 21 & 93KAT10M-1 & Khl-ebarr & 0.57 & 11.9 & & 2.67 & SS-mltgy, fgr,vwsrtd,rnd,vwcmt,qtz,calc,dk mafic gr & \\
\hline 18 & 93KAT10N-1 & Khl-ebarr & 0.55 & 11.8 & & 2.67 & SS-mltgy,fgr,vwsitd, rnd,vwcmt,qtz,calc,dk mafic gr & \\
\hline 74 & 93KAT110A-1 & $\mathrm{Ks}$ & 0.02 & 12.9 & & 2.72 & & \\
\hline 62 & 93KAT110A-2 & Ks & 2.42 & 9.0 & & 2.68 & & \\
\hline 79 & 93KAT111A-1 & $\mathrm{Kk}$ & $<0.01$ & 3.9 & & 2.72 & & \\
\hline 54 & 93KAT112/A-1 & Ks & 0.31 & 12.1 & & 2.73 & & \\
\hline 87 & 93KAT112A-4 & & & NOPLUG & & & & \\
\hline 16 & 93KAT11A-1 & Khl-ebarr & 0.23 & 16.1 & & 2.68 & SS-brngy,vf-vcgr,psitd,sbrnd,wcmt,qtz,occ calc \& mic & \\
\hline 14 & 93KAT11A-2 & Khl-ebarr & 0.07 & 17.4 & & 2.67 & SS-mltgy,vf-cgr,predfgr,wsrtd,sbrnd,vwcmt,qtz,dk mafic gr,occ calc & \\
\hline 17 & 93KAT11A-4 & Khl-ebarr & 2.81 & 19.9 & & 2.67 & SS-ltgy,vf-fgr,predfgr,wsrtd,rnd,wcmt,qtz,occ dk mafic gr \& calc & \\
\hline 23 & 93KAT11B-1 & Khl-ebarr & 0.13 & 12.0 & & 2.64 & SS-ltgy,fgr,vwsrtd,sbrnd,wcmt, qtz, calc, dk mafic gr & \\
\hline 4 & 93KAT11C-1 & Khl-ebarr & 0.96 & 15.9 & & 2.61 & SS-Itgy,vf-mgr,predfgr,wsrtd, rnd, vwcmt,qtz,dk arg gr,occ calc & \\
\hline 25 & 93KAT13C-1 & $\mathrm{Kk}$ & $<0.01$ & 1.4 & & 2.72 & Ls-mdkgy,hd,mass, gran & \\
\hline 46 & 93KAT15A-1 & Kk & 0.02 & 11.0 & & 2.74 & SS-brngy,vf-fgr,predvfgr,wsrtd, rnd, wcmt, qtz, dk mafic gr,mic & \\
\hline 48 & 93KAT183-1 & & 0.24 & 3.3 & & 2.74 & & \\
\hline 50 & 93KAT18A-1 & & 0.01 & 11.8 & & 2.76 & & \\
\hline 10 & 93KAT1A-2 & $\mathrm{Jn} ?$ & $<0.01$ & 7.6 & & 2.75 & SS-mdkgy,vf-mgr,predfgr,wsrtd,rnd,vwcmt,qtz,dk mafic gr,occ pyr,tr $h$ & hem \\
\hline 3 & 93KAT1D-2 & $\mathrm{Kp}$ & 0.19 & 14.9 & & 2.67 & SS-ltolvgy,vf-mgr,predfgr, wsrtd, rnd,vwcmt, qtz,chlor,occ brn mic,tr cal & \\
\hline 71 & 93KAT20A-1 & $\mathrm{Jn}$ & 0.26 & 4.4 & & 2.59 & & \\
\hline 57 & 93KAT21A-1 & $\mathrm{Jn}$ & 469.22 & 9.4 & & 2.64 & FRAC & \\
\hline 58 & 93KAT22A-1 & $\mathrm{Jn}$ & 1.25 & 6.2 & & 2.67 & & \\
\hline 69 & 93KAT26/A-1 & $\mathrm{Jn}$ & 0.02 & 5.1 & & 2.54 & & \\
\hline 5 & 93KAT3A-2 & Kcamp & 0.01 & 3.8 & & 2.78 & SS-ltgy,vf-fgr,predvfgr,vwsrtd,rnd,vwcmt,qtz,calc,occ dk arg gr,sid & \\
\hline 7 & 93KAT3A-4 & Kcamp & 0.61 & 6.4 & & 2.72 & SS-ltgy,vf-mgr,predfgr,wsrtd, rnd,vwcmt,qtz,dk arg gr,calc,tr sid & \\
\hline 43 & 93KAT4B-2 & $\mathrm{Kk}$ & 0.48 & 5.1 & & 2.73 & SS-Itgy,vf-vcgr,predmgr,psitd, sbang,vwcmt,qtz,calc, dk mafic gr & \\
\hline 6 & 93KAT6B-1 & Klcamp & 0.28 & 16.3 & & 2.69 & SS-ltgy,f-cgr,predmgr, wsrtd,rnd,vwcmt,qtz, dk mafic gr,occ calc & \\
\hline 9 & 93KAT6C-2 & Klcamp & 0.04 & 13.2 & & 2.68 & SS-mltgy,vf-mgr,predfgr,wsrtd,rnd,vwcmt,qtz, dk arg gr,tr calc & \\
\hline 35 & 93KAT6C-5 & Kemaes & 0.02 & 12.3 & & 2.70 & SS-ltgy,f-cgr,predmgr, wsrtd, sbrnd,vwcmt,qtz, dk mafic gr,lim,calc & \\
\hline 44 & 93KAT8A-1 & $\mathrm{Jn}$ & 1.24 & 8.4 & & 2.64 & SS-Itgy,vf-mgr,predfgr,wsrtd,sbrnd,wcmt,qtz,dk mafic gr,chlor & \\
\hline 39 & 93KAT8B-1 & $\mathrm{Jn}$ & 1.43 & 6.7 & & 2.64 & SS-ltgy,f-cgr,predmgr,wsitd,sbrnd,vwcmt, qtz, dk mafic gr,chlor & \\
\hline 40 & 93KAT8C-1 & $\mathrm{Jn}$ & 0.02 & 5.6 & & 2.66 & SS-ltbrngy,vfgr,vwsrtd, sbrnd, vwcmt,qtz,dk mafic gr,chlor & \\
\hline 38 & 93KAT8D-1 & $\mathrm{Jn}$ & 0.72 & 7.0 & & 2.64 & SS-ltgy,f-cgr,predmgr,wsrtd,sbrnd,vwcmt,qtz, dk mafic gr,calc,occ chlo & \\
\hline 41 & 93KAT8E-1 & $\mathrm{Jn}$ & 0.76 & 5.9 & & 2.67 & SS-mltgy,f-cgr,predmgr, wsttd,sbrnd,wcmt,qtz,chlor,calc,dk mafic gr & \\
\hline 37 & 93KAT8H-1 & $\mathrm{Jn}$ & 1.29 & 6.5 & & 2.64 & SS-ltgy,f-cgr,predmgr,wsrtd,sbrnd,vwcmt, qtz,chlor, mic & \\
\hline
\end{tabular}




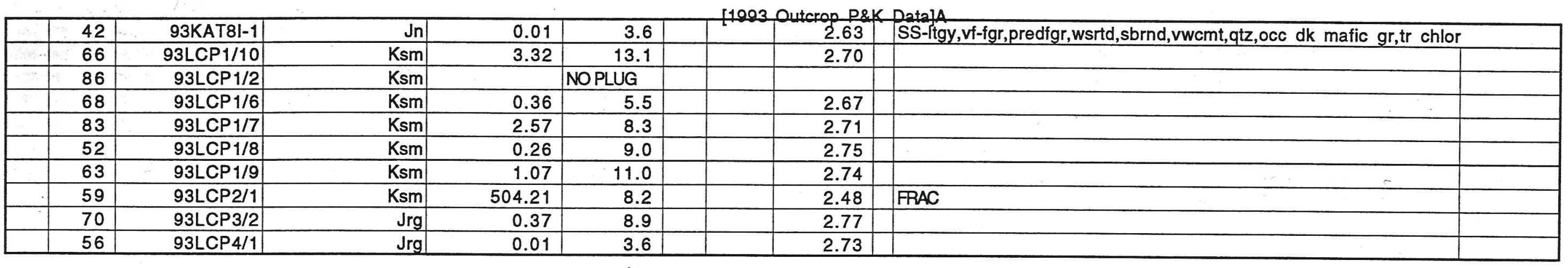




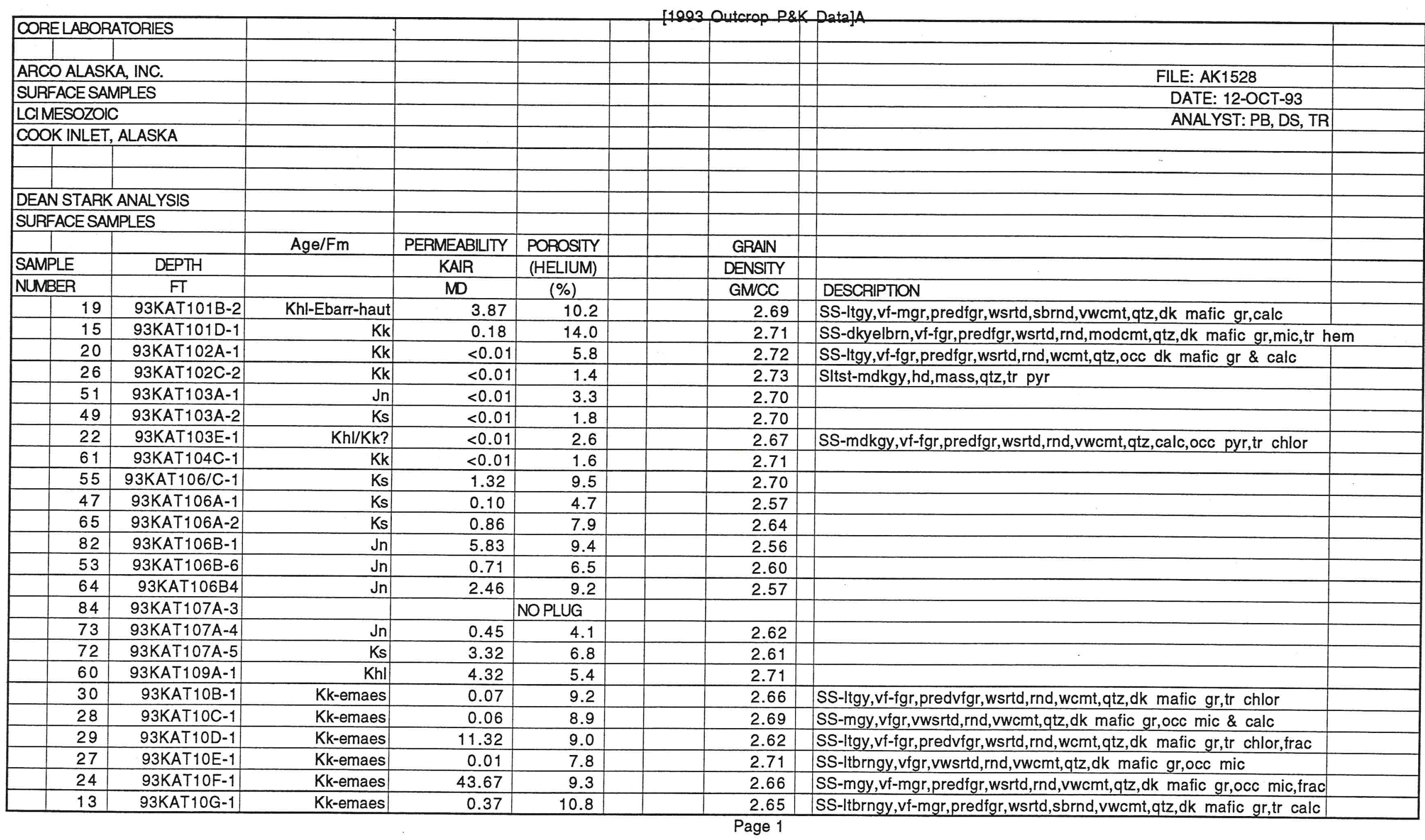




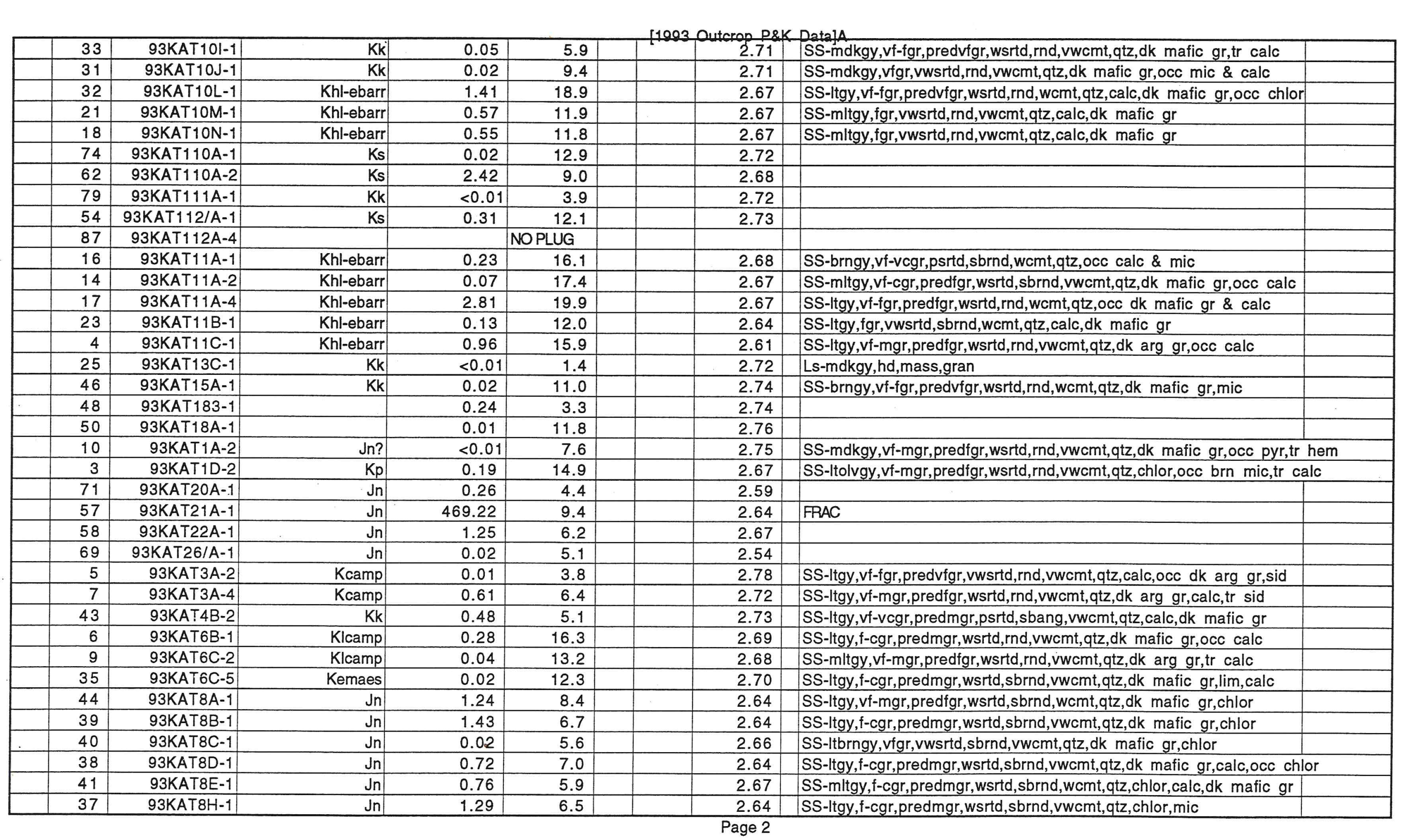




\begin{tabular}{|c|c|c|c|c|c|c|c|c|}
\hline & 42 & 93KAT8I-1 & $\mathrm{Jn}$ & 0.01 & 3.6 & 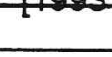 & 2.63 & SS-ltgy,vf-fgr,predfgr,wsrtd,sbrnd,vwcmt,qtz,occ dk mafic gr,tr chlor \\
\hline+ & 66 & 93LCP1/10 & $\mathrm{Ksm}$ & 3.32 & 13.1 & & 2.70 & \\
\hline & 86 & $93 L C P 1 / 2$ & $\mathrm{Ksm}$ & & NO PLUG & & & \\
\hline & 68 & 93LCP $1 / 6$ & $\mathrm{Ksm}$ & 0.36 & 5.5 & & 2.67 & \\
\hline & 83 & 93LCP $1 / 7$ & $\mathrm{Ksm}$ & 2.57 & 8.3 & & 2.71 & \\
\hline & 52 & 93LCP $1 / 8$ & $\mathrm{Ksm}$ & 0.26 & 9.0 & & 2.75 & \\
\hline & 63 & 93LCP1/9 & Ksm & 1.07 & 11.0 & & 2.74 & c \\
\hline & 59 & 93LCP2/1 & Ksm & 504.21 & 8.2 & & 2.48 & FAC \\
\hline & 70 & 93LCP3/2 & $\mathrm{Jrg}$ & 0.37 & 8.9 & & 2.77 & \\
\hline & 56 & $93 \mathrm{LCP} 4 / 1$ & $\mathrm{Jrg}$ & 0.01 & 3.6 & & 2.73 & \\
\hline
\end{tabular}


COASTAL

SCIENCE

LABORATORIES, INC.

6000 Mountain Shadows Drive - Austin, Texas 78735 • (512) 288-5533

October 8, 1993

Arco Oil and Gas Company

Attn: Mr. Chuck Roberts

2300 West Plano Parkway

Plano, TX 75075

Dear Mr. Roberts:

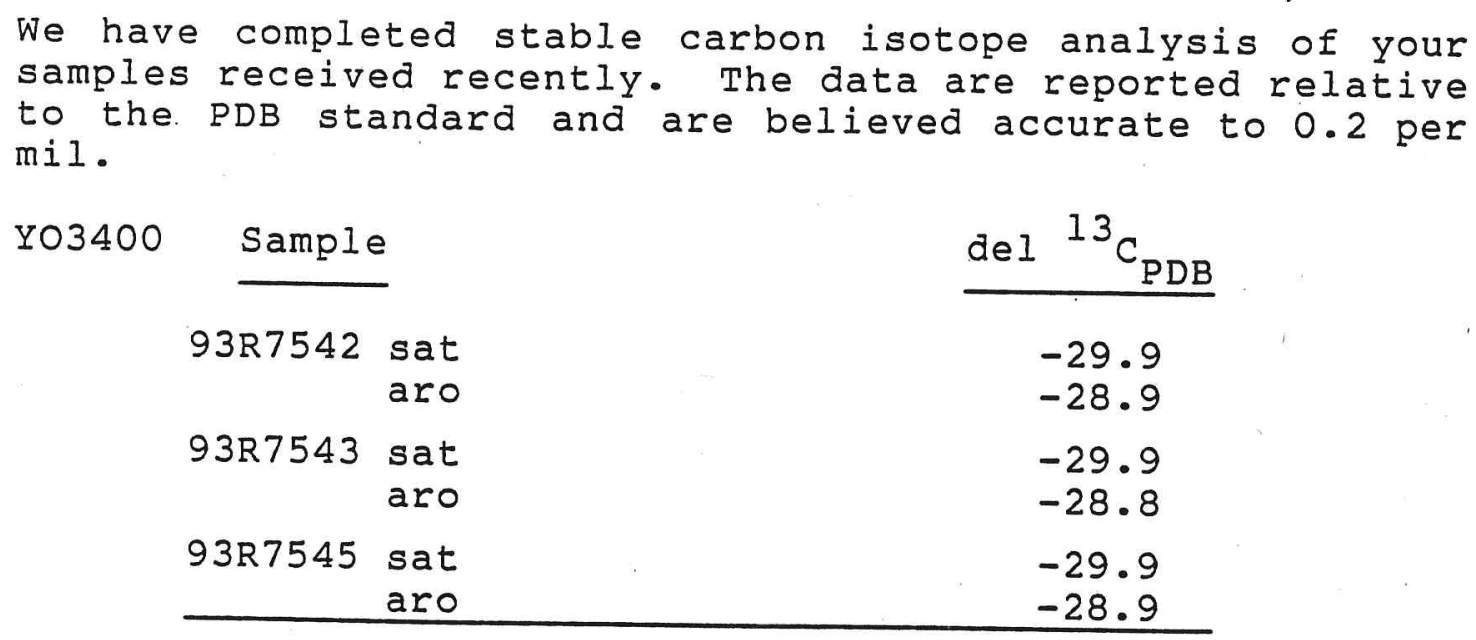

Yours truly,

Kenneth Winters

Laboratory Manager

$\mathrm{KW}: \mathrm{Ip}$

CSL Ref:ATO 


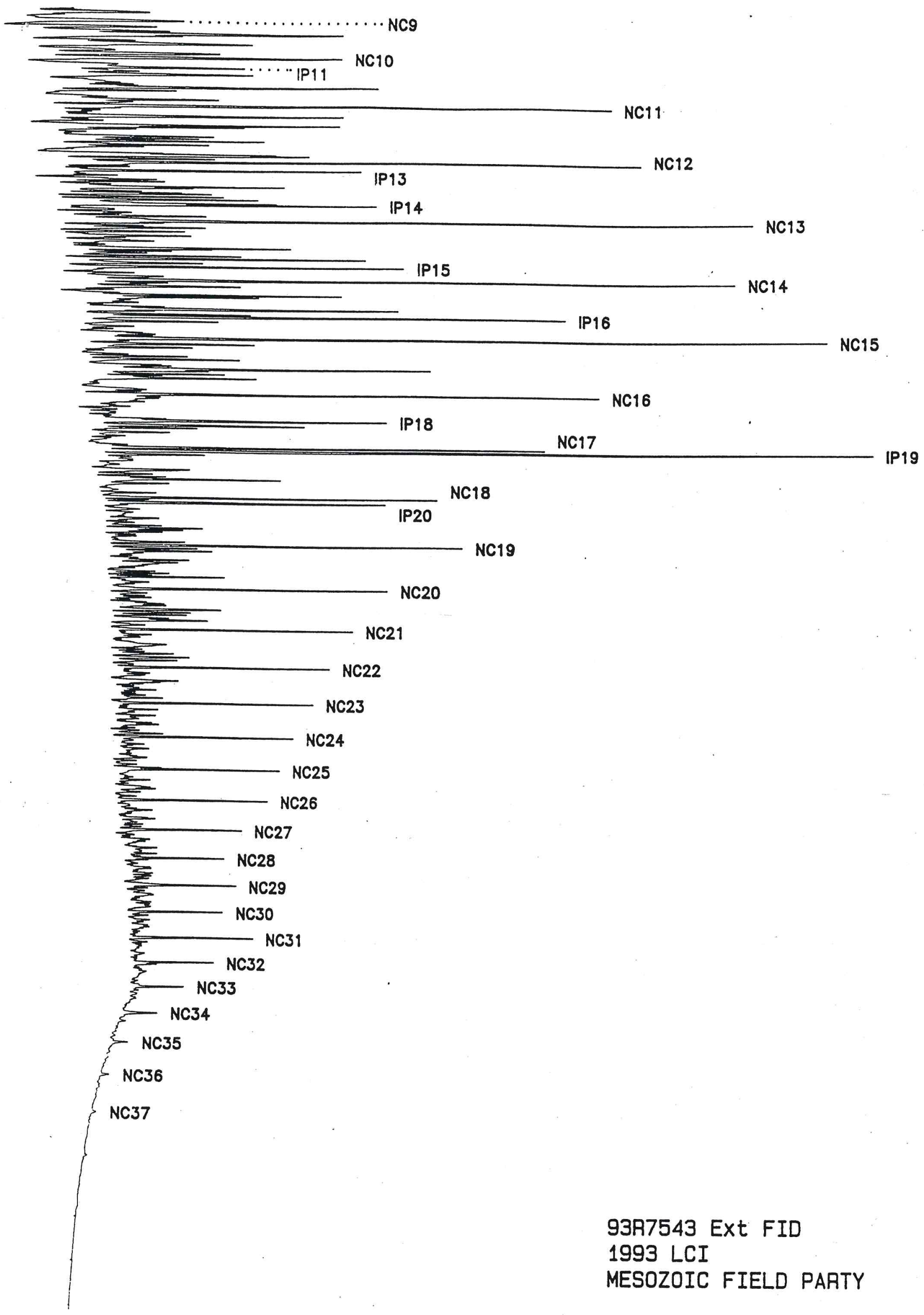


NORMAL PARAFFINS

AREA\%

ISOPRENOIDS

20.876

RESOLVED UNKNOWNS

9.107

70.016

\begin{tabular}{|c|c|}
\hline NAME & $A R E A \%$ \\
\hline $\mathrm{NC9}$ & 1.194 \\
\hline NC1D & 1.368 \\
\hline I P 11 & .907 \\
\hline$N C 11$ & 1.646 \\
\hline$N C 12$ & 1.551 \\
\hline I P 13 & .934 \\
\hline$I P \perp 4$ & .597 \\
\hline NC13 & 1.903 \\
\hline IP 15 & .898 \\
\hline NCl 14 & 1.940 \\
\hline IF 16 & 1.366 \\
\hline$N C 15$ & 1.964 \\
\hline NC15 & 1.438 \\
\hline IP18 & .985 \\
\hline NC17 & 1.100 \\
\hline IP 19 & 2.393 \\
\hline$N C 18$ & .762 \\
\hline IP 20 & .928 \\
\hline NC19 & .75 \\
\hline
\end{tabular}

NAME

AREA\%

NC2O

.677

NC21 $\quad .555$

NC22 $\quad .466$

$\mathrm{NC23} \quad .435$

NC24

NC25 $\quad .361$

NC26

NC27 $\quad .341$

NC28

NC29 $\quad .371$

NC30 $\quad .226$

NC31 $\quad .297$

NC32 $\quad .177$

NC33 $\quad .134$

NC34

NC35

NC36 $\quad .031$

$\mathrm{NC37}$ 
REQUESTED CALCULATIONS USING AREA PERCENT VALUES FROM:

SAMPLE: $93 R 7543$

RESULT FILE: E93R7543.RES

RATIOS:

$\begin{array}{lll}\text { PRISTANE / PHYTANE } & = & 2.580 \\ \text { NC17/IP19 } & = & .459 \\ \text { NC18/IP20 } & = & .821 \\ \text { NC18/NC19 } & = & 1.004\end{array}$

$C P I=$ 1.219

NORMALIZATION OF NC17, IP19, AND NC21:

$N C 17=27.167$

IP19 $=59.124$

$N C 21=13.709$

GENERAL FILE NAME: E93R7543. GEN 


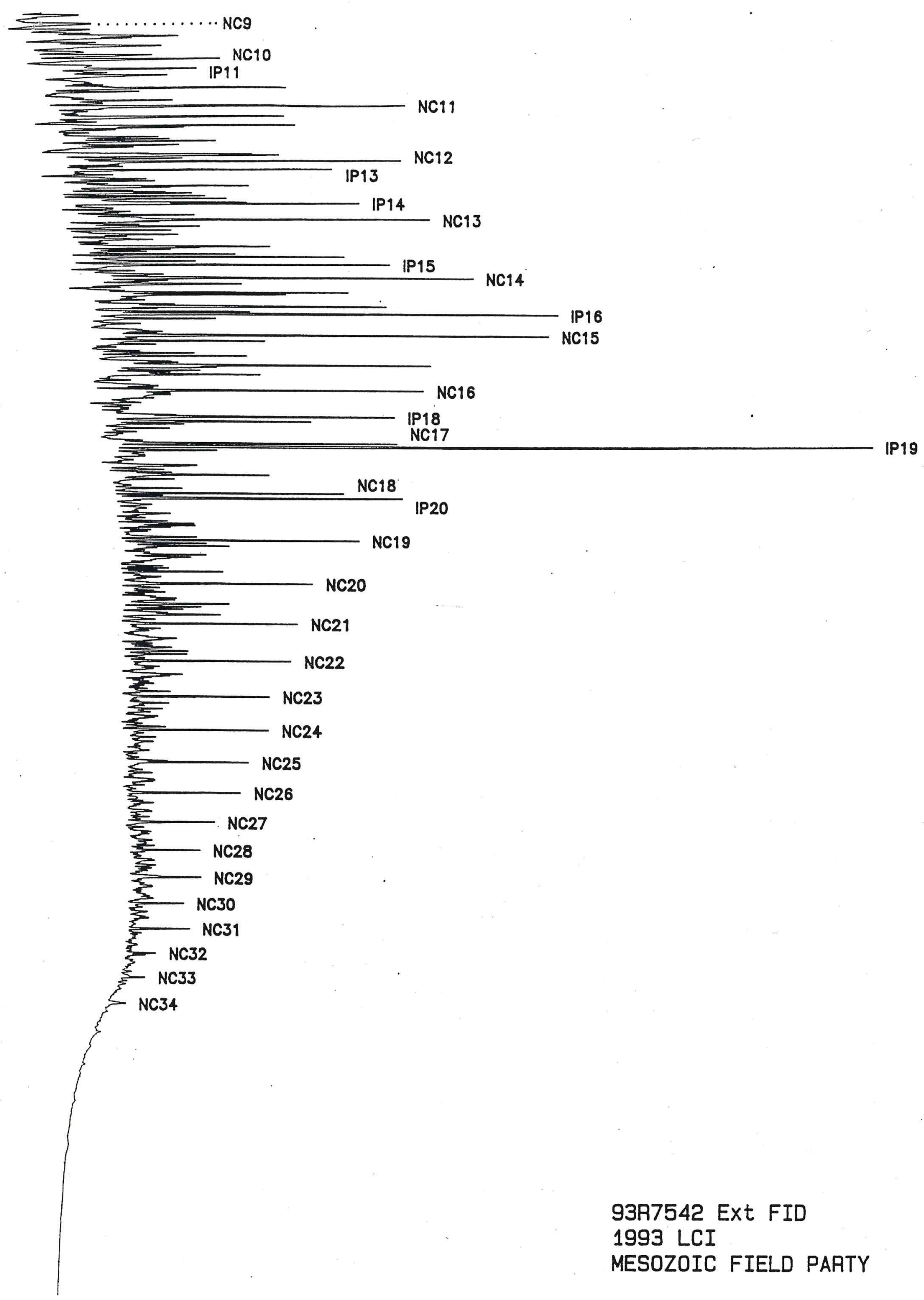


SAMPLE: 93R7542

NORMAL PARAFFINS

ISOPRENOIDS

RESOLVED UNKNOWNS

NAME

NC9

NC10

I P 11

$N C 11$

$N C 12$

IF 13

I P 14

NC 13

IF 15

NCl 4

IF 16

NC15

NC1G

I $P 18$

$\mathrm{NC} 17$

IPIS

NC16
AREA\%

.720

1.021

.321

1. 319

1. 362

1. 000

.787

1. 189

1.056

1. 631

1. 551

1. 635

1. 270

1. 189

.788

2. 999

.618

RESULT FILE: E93R7542.RES

AREA\%

16.767

10.529

72.704

NAME

I P 20

NC19

NC2O

NC2!

NC2 2

NC2 3

NC2 4

NC2 5

NC2

NC2 7

NC28

NC29

$\mathrm{NC} 3 \mathrm{O}$

NC3 1

$\mathrm{NC} 32$

NC3 3

$\mathrm{NC} 34$
AREA\%

1. 127

.611
.552

.513

.453

.398

.419

.336

.314

.303

.181

.301

.156

.176
.088

.097

.118

$\therefore+\cdots$ 
REQUESTED CALCULATIONS USING AREA PERCENT VALUES FROM:

SAMPLE: $93 R 7542$

RESULT FILE: E93R7542.RES

RATIOS:

PRISTANE / PHYTANE $=2.661$

NC17 / IP19 = .330

NC18/IF20 = . 548

NC18/NC19= $=1.011$

$C P I=\quad 1.233$

NORMALIZATION OF NC17, IP19, AND NC21:

$N C 17=21.963$

IP19 $=66.637$

$N C 21=11.399$

GENERAL FILE NAME: E93R7542.GEN 


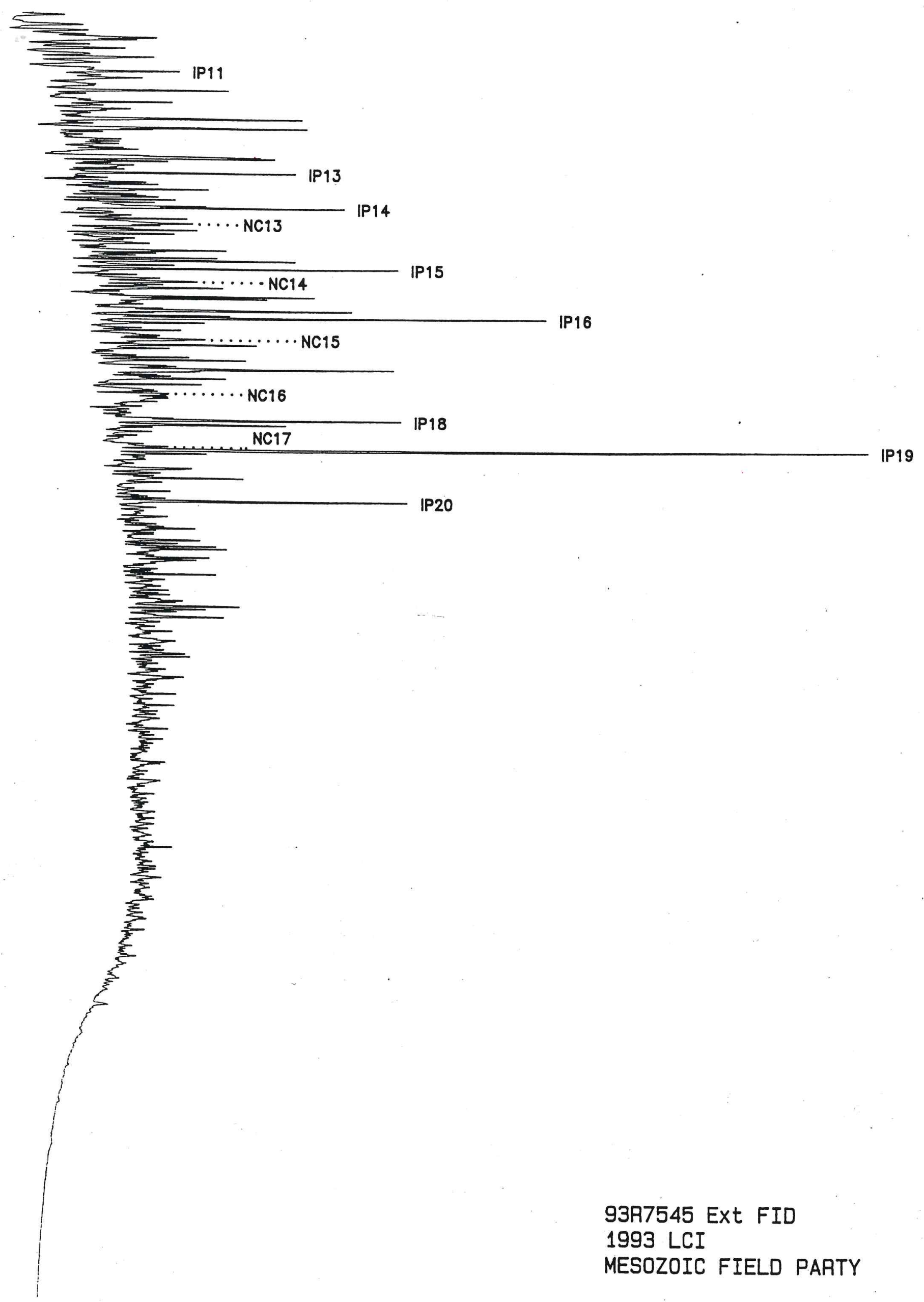


NORMAL PARAFFINS

ISOPRENOIDS

RESOLVED UNKNOWNS
AREA\%

3.361

11.610

85.029

NAME

AREA\%

NAME

AREA\%

IP 11

.434

1.070

.906

.966

1.193

.367

1.695
NC 15

NC15

IP 13

NC17

IP 19

IP 20
.868

.215

1.405

.345

3.544

1. 364
NC 14

1.695 
REQUESTED CALCULATIONS USING AREA PERCENT VALUES FROM:

SAMPLE: 93F75 75

RESULT FILE: E93R7545.RES

RATIOS:

$\begin{array}{llr}\text { PRISTANE / PHYTANE } & \text { r } \\ \text { NC17/IP19 } & 2.599 \\ \text { NC18/IP10 } & =097 \\ \text { NC18/NC19 } & =* * * * * * *\end{array}$

$C P I=$

NORMALIZATION OF NC17, IP19, AND NC21:

$N C 17=8.874$

$I P 19=91.125$

$N C 21=0.000$

GENERAL FILE NAME: E93R7545. GEN 
COASTAL

(5) SCIENCE

LABORATORIES, INC.

6000 Mountain Shadows Drive - Austin, Texas 78735 • (512) 288-5533

October 8, 1993

Arco Oil and Gas Company

Attn: Mr. Chuck Roberts

2300 West Plano Parkway

Plano, TX 75075

Dear Mr. Roberts:

We have completed stable carbon isotope analysis of your samples received recently. The data are reported relative to the PDB standard and are believed accurate to 0.2 per mil.

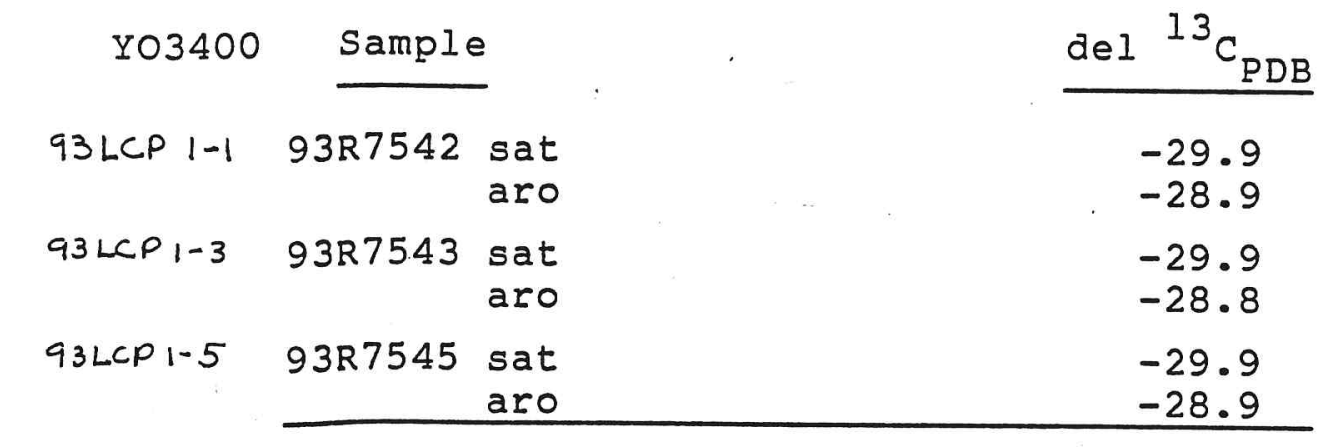

Yours truly,

Kenneth winters

Laboratory Manager

$\mathrm{KW}: 1 \mathrm{p}$

CSL Ref:ATO 


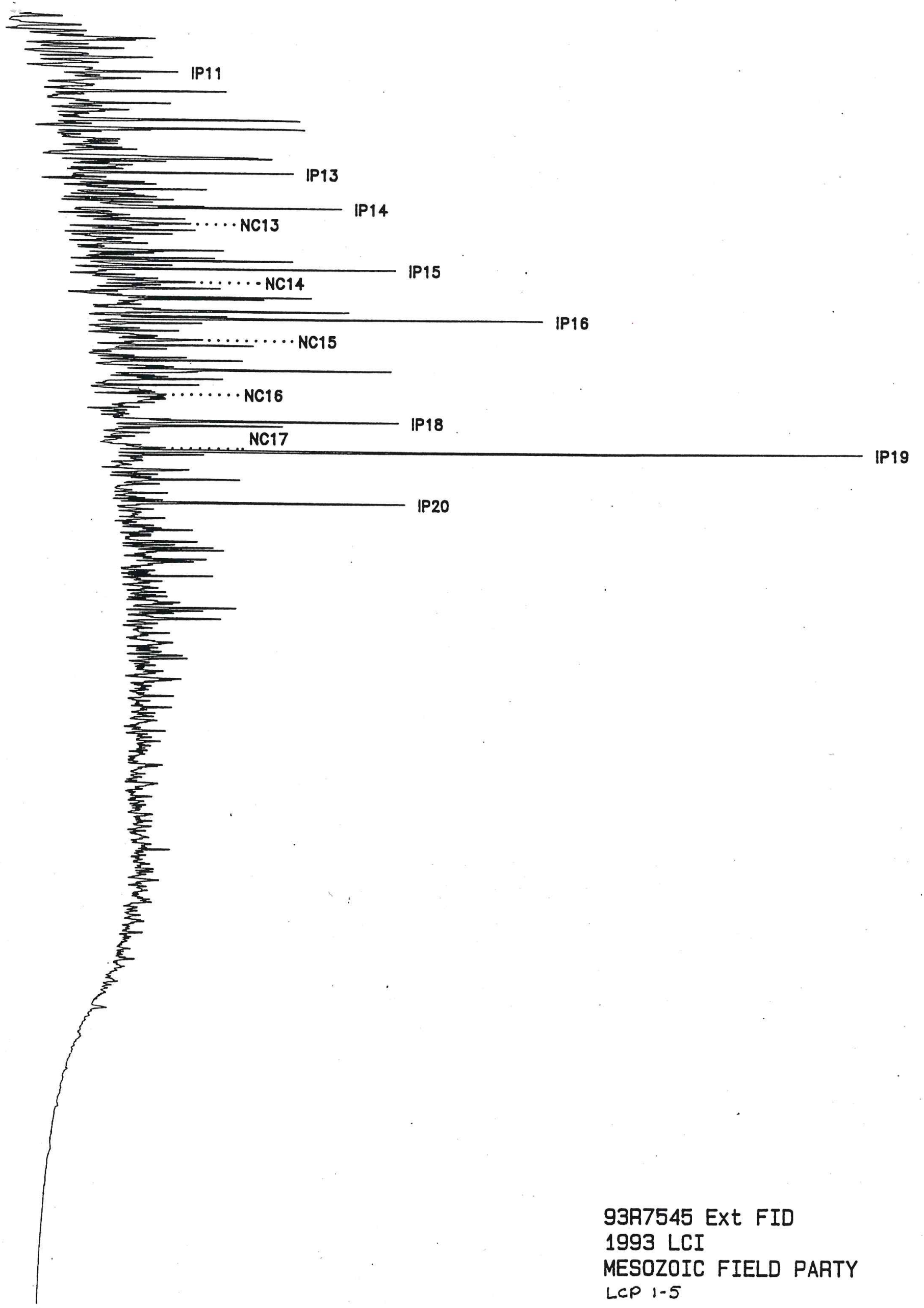




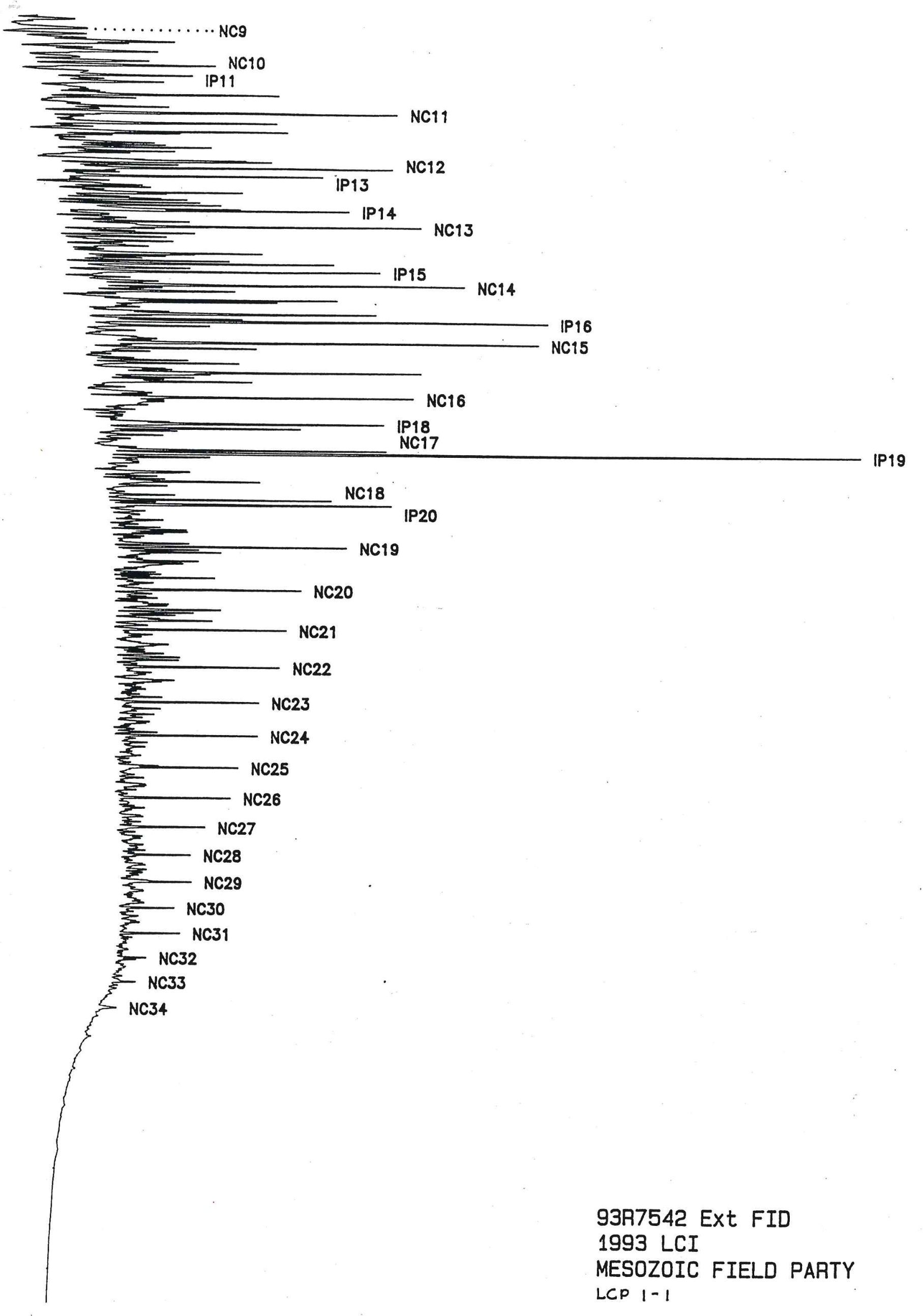




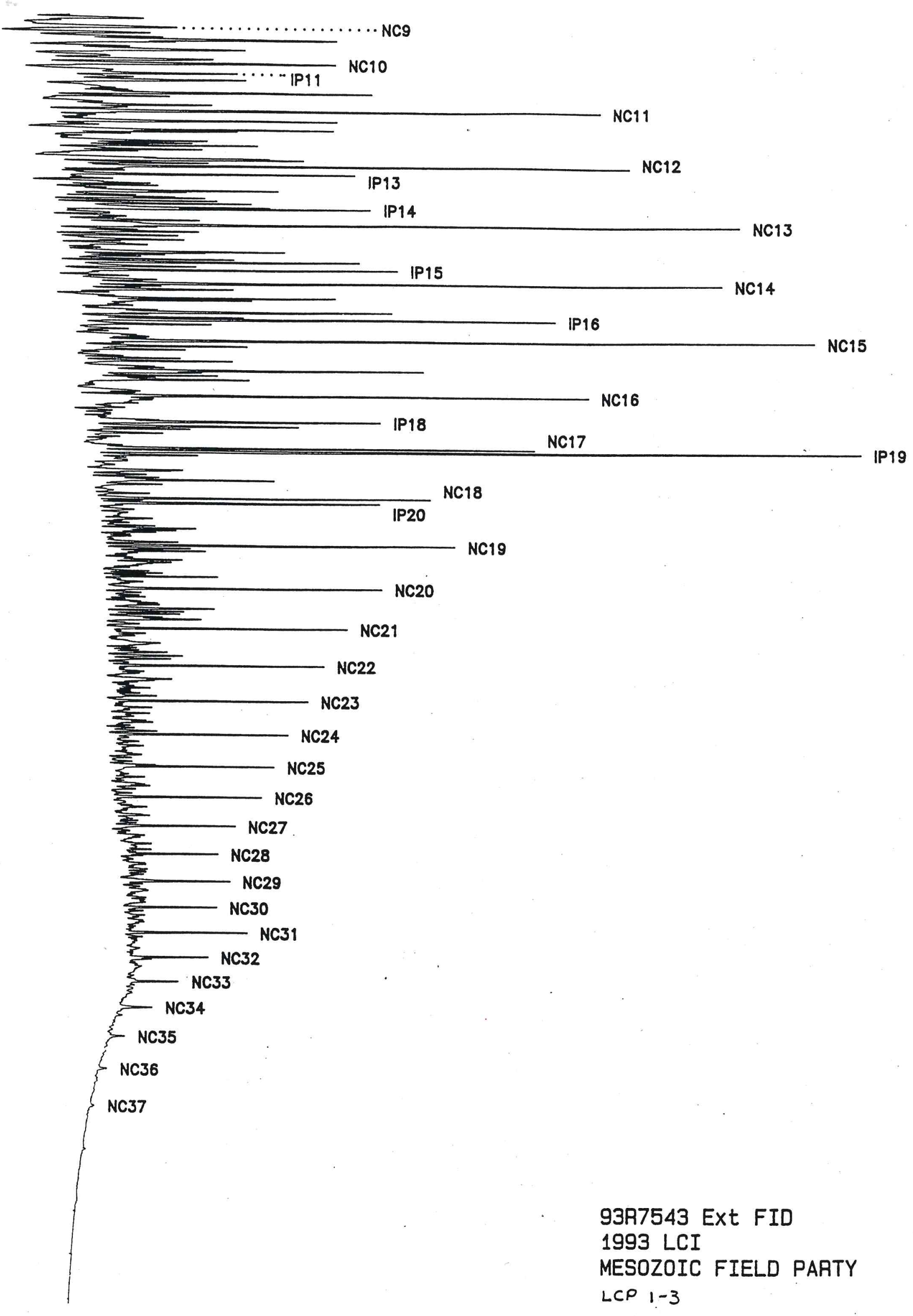




\begin{tabular}{|c|c|c|c|c|c|c|c|c|c|c|c|c|c|c|c|c|c|c|c|}
\hline Stop : & Spl & Purpose & Degs & g. Min & Sec. & Latitude & Deg. & & & Longitude & Geographic Area & Sen & Twp & N-\{R & $\operatorname{lng} E$ & Quad. & Fm. & Coll. By & \\
\hline & & All pabo,pay, otc. w & ill be & proce & $\operatorname{ssed}$ to & tor pay and tor & & & & & & & & & & & & & \\
\hline \begin{tabular}{|l} 
93KAT106B \\
93KAT166C
\end{tabular} & 5 & VIT & 59 & 14 & 34 & 59.07611 & 153 & 45 & 39 & 153.760833 & River Island & 3 & 13 & 2 & & | & Jn & TDV,BM & \\
\hline \begin{tabular}{|l|} 
93KAT106C \\
93KAT10H
\end{tabular} & 4 & VIT & 59 & 4 & 28 & 59.07444 & 153 & 44 & 51 & 153.7475 & Douglas River Island & 2 & 13 & 52 & & V ILIAMNA (A-1\&2) & Ks & DD,SK & \\
\hline 93KAT10H & $\frac{2}{2}$ & VITRINIIE & $\frac{58}{58}$ & 50 & 47 & 58.84639 & $\frac{153}{1.53}$ & \begin{tabular}{|l|l|}
56 \\
57
\end{tabular} & 40 & $\frac{153.944444}{152955}$ & W. DOUGLAS RIVER & 28 & 15 & 52 & & VAFOGNAK (DG) & Kk & GVK,SK,RT,RK & \\
\hline 93KAT11A & 3 & VITRINITE & 58 & 51 & $\frac{1}{7}$ & \begin{tabular}{|l|l|}
58.055190 \\
58.04
\end{tabular} & 153 & 57 & 51 & \begin{tabular}{|l|}
153.955 \\
153.964167 \\
\end{tabular} & \begin{tabular}{|l} 
W. DOUGLAS RIIER \\
W. DOULAS RIVER
\end{tabular} & $\frac{28}{28}$ & $\frac{15}{15}$ & \begin{tabular}{|l|l|}
$\mathrm{s}$ & 2 \\
$\mathrm{~s}$ & 2 \\
\end{tabular} & & AFOGNAK (D6) & Kk & GVK,SK,RT,RK & \\
\hline 93KATIA & 1 & VITRINITE & 58 & 0 & 9 & 58.0025 & 154 & 47 & & 154.785556 & MT. PEDMAR & 24 & 25 & $\begin{array}{ll}5 \\
5\end{array}$ & & $\begin{array}{l}\text { AFOGNAK (DE) } \\
\text { MT. KATMAI (A3) }\end{array}$ & \begin{tabular}{|l|l}
$\mathrm{Kk}$ \\
$\mathrm{Jn} ?$
\end{tabular} & GVK & \\
\hline 93KAT1D & 1 & VITRINITE & 58 & 0 & 7 & 58.00194 & 154 & 46 & 41 & 154.778056 & MT. PEDMAR & 19 & 25 & $\begin{array}{lll}5 & 3 \\
\end{array}$ & & VIT. KATMAI (A3) & Kk? & GVK & \\
\hline 93KAT3A & 3 & VITPINITE & 58 & 0 & 46 & 58.01278 & 154 & 44 & 15 & 154.7375 & MT. PEDMAR & 17 & 25 & \begin{tabular}{|l|lll}
5 & 3
\end{tabular} & & MT. KATMAI (A3) & $\mathbf{K k}$ & & \\
\hline 93KAT4B & 1 & VIRIINTIE & 58 & 1 & 47 & 58.02972 & 154 & 36 & 39 & 154.610833 & CAPE ILKTUGTAK & 7 & 25 & \begin{tabular}{|l|l|l|l}
$\mathbf{s}$ & 3 \\
\end{tabular} & $3 w$ & MT. KATMAI (A2) & Kk & GVK & \\
\hline 93KATGC & 3 & VIT. & 58 & to & 9 & 58.0025 & 154 & 46 & 15 & 154.770833 & MT. PEDMAR & 17 & 25 & & \begin{tabular}{l|l}
$3 \mathrm{~W}$ \\
\end{tabular} & MT. KATMAI (A3) & Kp/Kk? & GVK & \\
\hline 93KATBG & $1 \mathrm{~A}$ & VITRINITE & 58 & 33 & 22 & 58.55611 & 154 & & 26 & 154.107222 & S.KAGUYAK CRATER & 5 & 19 & \begin{tabular}{l|l}
$s$ & 2 \\
\end{tabular} & & \begin{tabular}{|l} 
MT. KATMAI (C1) \\
\end{tabular} & Jn & GVK & \\
\hline 93LCP1 & & EXIRACT & 59 & 58 & 57 & 59.9825 & 152 & 41 & 51 & 152.6975 & SADDLE MOUNTAIN & 27 & 2 & \begin{tabular}{|l|l|l|l|l} 
& 2 \\
\end{tabular} & & SELDOVIA (D-8) & Ksm & TDV,SK,DD,BM & \\
\hline $93 L C P 1$ & 3 & EXTRACT & 59 & 58 & 57 & 59.9825 & 152 & 41 & 51 & 152.6975 & SADDLE MOUNTAIN & 27 & $\sqrt{2}$ & 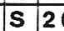 & & SELDOVIA (D-8) & Ksm & TDV,SK,DD,BM & \\
\hline 93LCP1 & 4 & EXIRACT & 59 & 58 & 57 & 59.9825 & 152 & 41 & 51 & 152.6975 & SADDLE MOUNTAIN & 27 & 2 & \begin{tabular}{|l|l|l|l|l|}
522 \\
\end{tabular} & & ISELDOVIA (D-8) & Ksm & TDY,SK.DD,BM & \\
\hline 93LCP1 & 5 & EXTRACT & 59 & 58 & 57 & 59.9825 & 152 & 41 & 51 & 152.6975 & SADDLE MOUNTAIN & 27 & 2 & $15 \mid 2$ & & ISELDOVIA (D-8) & $K_{\mathbf{3 m}}$ & TDY SKDDBMM & \\
\hline $93 \mathrm{LCP} 4$ & 2 & PALEO,GEOCHEM & 60 & 2 & 45 & 60.04583 & 152 & 53 & 55 & \begin{tabular}{|l|}
152.898611 \\
\end{tabular} & \begin{tabular}{|l} 
RED GLACIER \\
\end{tabular} & 33 & 1 & & $1 w$ & KENA) (A-8) & Jra & DOSKTRYRY & \\
\hline 93LCP4 & 3 & PALEO,GEOCHEM & 60 & 2 & 45 & 60.04583 & 152 & 53 & 55 & 152.898611 & RED GLACIER & 33 & 1 & 52 & & KENAI (A-8) & Jira & DDSKFTPYBM & \\
\hline 93LCP4 & & PALEO,GEOCHEM & 60 & 2 & 45 & 60.04583 & 152 & 53 & 55 & 152.898611 & RED GLACIER & 33 & 1 & s 2 & $1 \mathrm{w}$ & KENAI (A-8) & Jrg & DD,SK,TDV,BM & \\
\hline 93LCP4 & 5 & PALEO,GEOCHEM & 60 & 2 & 45 & 60.04583 & 152 & 53 & 55 & 152.898611 & RED GLACIER & 33 & 1 & \begin{tabular}{|l|l|l|l|}
5 & 2 \\
\end{tabular} & $1 \mathrm{w}$ & KENAI (A-8) & Jrg & $\mathrm{DD}, \mathrm{SK}, \mathrm{TDV}, \mathrm{BM}$ & \\
\hline 93LCP4 & 6 & PALEO,GEOCHEM & 60 & $T_{0}$ & 45 & 60.04583 & 152 & 53 & 55 & 152.898611 & RED GLACIER & 33 & & s 2 & & KENAI (A-8) & Jrg & DD,SK,TDV,BM & \\
\hline $93 \mathrm{LCP} 4$ & & PALEO,GEOCHEM & 60 & 2 & 45 & 60.04583 & 152 & 53 & 55 & 152 & RED GLACEER & 33 & 1 & 52 & $\mathrm{w}$ & KENAI (A-8) & Jrg & DD,SK,TDV,BM & \\
\hline 93LCP4 & 8 & PALEO,GEOCHEM & 60 & 2 & 45 & 60.04583 & 152 & 53 & 55 & 152.898611 & RED GLACIER & 33 & 1 & \begin{tabular}{|l|l}
5 \\
\end{tabular} & $1 \mathrm{w}$ & KENAI (A-8) & Jrg & DD,SK,TDV,BM & \\
\hline 93LCP4 & & PALEO,GEOCHEM & 60 & 2 & 45 & 60.04583 & 152 & 53 & 55 & 152.898611 & AED GLACIER & 33 & 1 & \begin{tabular}{|l|l|l|l|}
5 & 2 \\
\end{tabular} & $1 \mathrm{w}$ & KENAI $(A-8)$ & Jrg & DD,SK.TDV.BM & \\
\hline 3 3LCP4 & 10 & PALEO,GEOCHEM & 60 & 2 & 45 & 60.04583 & 152 & 53 & 55 & 152. & RED GLACIER & 33 & 1 & \begin{tabular}{|l|l|l}
$\mathbf{s}$ & 2 \\
\end{tabular} & $1 \mathrm{~W}$ & KENAI (A-8) & Jrg & DD.SK.TDV.BM & \\
\hline 93LCP4 & 11 & PALEO,GEOCHEM & 60 & 2 & 45 & 60.04583 & 152 & 53 & 55 & 152.898611 & RED GLACER & 33 & 1 & \begin{tabular}{l|l}
$\mathbf{s} 2$ \\
\end{tabular} & \begin{tabular}{l|l}
$1 \mathrm{w}$ \\
\end{tabular} & KENAI (A-8) & Jirg & DDSK TDYBH & \\
\hline 93LCP4 & 12 & PALEO,GEOCHEM & 60 & 2 & 45 & 60.04583 & 152 & 53 & 55 & 152.898611 & RED GLACIER & $\mathbf{3 3}$ & 1 & $\begin{array}{lll}52 & \end{array}$ & $\bar{w}$ & KENAI (A-8) & $J n$ & DDSKTDVPA & \\
\hline 93LCP4 & 13 & PALEO,GEOCHEM & 60 & 2 & 45 & 60.04583 & 152 & 3 & 55 & 152.898611 & RED GLACEFP & 33 & & $s$ & & KENAL (A-8) & Jra & DDSKTTYRAM & \\
\hline 93LCP4 & 14 & PALEO,GEOCHEM & 60 & & 45 & 60.04583 & 52 & 53 & 55 & 152.898611 & RED GLACIER & 33 & & s & & $\mathrm{KEN}$ & $\mathrm{Jr}$ & V.BM & \\
\hline 93LCP4 & 15 & PALEO,GEOCHEM & 60 & $\mathbf{2}$ & 45 & 50.04583 & 52 & $=2>0>3$ & 55 & $52 . \varepsilon$ & RED GLACIER & 33 & & 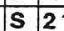 & & KENA & 舟 & DD, & \\
\hline 3LLP4 & 16 & PALEO,GEOCHEM & 60 & & 45 & .04583 & $=$ & & & 52. & RED GLACEER & & & \begin{tabular}{|l|l|}
$\mathrm{s}$ & 2 \\
\end{tabular} & & KENAI (A-8) & $\mathrm{Jrg}$ & \begin{tabular}{|l|}
$\mathrm{DD}, \mathrm{SK}, \mathrm{TDV}, \mathrm{BM}$ \\
\end{tabular} & \\
\hline
\end{tabular}




\begin{tabular}{|c|c|c|c|c|c|c|c|c|c|c|c|}
\hline Description \& Comments & Lab \# & $\mathrm{VR}$ & S1 & 52 & TOC & TMAX & & a & 53 & HAC & Stop \# \\
\hline DOUGLAS RIVER ISLAND SECTION & \begin{tabular}{|l|}
$93 R 7531$ \\
\end{tabular} & 0.29 & & & & & & - & & & 93КАТ106В \\
\hline \multirow{4}{*}{ DOUGLAS RIVER ISLAND SECTION } & $93 \mathrm{R} 7532$ & 0.43 & & & & & & & & & 93KAT106C \\
\hline & 93R7533 & 0.36 & & & & & & & & & 93KAT10H \\
\hline & $93 \mathrm{R} 7534$ & 0.44 & & & & & & & & & 93KAT10K \\
\hline & 93R7535 & 0.46 & & & & & & & & & 93KAT11A \\
\hline \multirow[t]{6}{*}{ KAT $=$ KATMAI AREA } & 93R7536 & 0.54 & & & & & & & & & 93KAT1A \\
\hline & 93R7537 & 0.71 & & & & & & & & & 93KATID \\
\hline & 9387538 & 0.67 & & & & & & & & & 93KAT3A \\
\hline & 93R7539 & 1.34 & & & & & & & & & 93KAT4B \\
\hline & 93R7540 & 0.72 & & & & & & & & & 93KATGC \\
\hline & 93R7544 & 0.59 & & & & & & & & & 93KAT8G \\
\hline 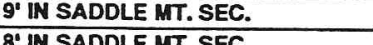 & & & & & & & & & & & $93 \mathrm{LCP} 1$ \\
\hline 8' IN SADDLE MT. SEC. & & & & & & & & & & & 93LCP1 \\
\hline \multirow{17}{*}{$20^{\circ}$ IN SADDLE MT. SEC. } & & & & & & & & & & & 93LCP1 \\
\hline & & & & & & & & & & & 93LCP1 \\
\hline & 93R8212 & 0.55 & 0.04 & 0.33 & & 499 & 34 & 54 & 0.52 & 4.1 & 93LCP4 \\
\hline & 93म8213 & 1 & 0.1 & 0.34 & 1.03 & 496 & 33 & 51 & 0.52 & 9.7 & $93 L C P 4$ \\
\hline & & No sample & & & & & & & & & $93 L C P 4$ \\
\hline & & No sampla & & & & & & & & & 93LCP4 \\
\hline & 93R8214 & 1.09 & 0.2 & 0.54 & 1.49 & 491 & 36 & 41 & 0.61 & 13.4 & $93 L C P 4$ \\
\hline & 93R8215 & 1.17 & 0.18 & 0.48 & 1.58 & 491 & 30 & \begin{tabular}{|l|l|}
37 \\
\end{tabular} & 0.59 & 11.4 & $93 \mathrm{LCP} 4$ \\
\hline & 93R8216 & 1.26 & 0.13 & 0.21 & 1.17 & 458 & 18 & 411 & 0.48 & 11.1 & 993LCP4 \\
\hline & 93R8217 & 1.3 & 0.03 & 0.17 & 0.35 & 490 & 49 & 140 & 0.49 & 8.6 & 993LCP4 \\
\hline & 93R8218 & 1.04 & 0.14 & 0.24 & 1.07 & 498 & 22 & 41 & $\mid \begin{array}{ll}0.44 \\
\end{array}$ & \begin{tabular}{|l|l|}
13.1 \\
\end{tabular} & 993LCP4 \\
\hline & 93R8219 & 1.22 & 0.02 & 0.14 & 0.83 & 499 & 17 & 87 & 0.72 & \begin{tabular}{|l|l|}
2.4 \\
\end{tabular} & 93LCP4 \\
\hline & 9388220 & 1 & 0.01 & 0.05 & 0.08 & 472 & 63 & \begin{tabular}{|l|l|}
438 \\
\end{tabular} & 0.35 & 12.5 & 93LCP4 \\
\hline & $93 \mathrm{R} 8221$ & 0.76 & 0.16 & 0.43 & 1.44 & 485 & 30 & \begin{tabular}{|l|l|l|l|}
44 \\
\end{tabular} & 0.63 & \begin{tabular}{|l|l|}
11.1 & \\
\end{tabular} & 93LCP4 \\
\hline & 93R8222 & 1.15 & 0.03 & 0.29 & 1.39 & 480 & 21 & 112 & 1.55 & 2.2 & $93 L C P 4$ \\
\hline & $93 \mathrm{RB} 223$ & 1.32 & 0.02 & 0.15 & 1.24 & 487 & 12 & 70 & $\begin{array}{l}0.87 \\
\end{array}$ & 1.6 & 93LCP4 \\
\hline & $93 \mathrm{R} 8224$ & 1.05 & 0.02 & 0.11 & 0.28 & 493 & 39 & 1111 & 0.31 & 7.1 & $93 L C P 4$ \\
\hline
\end{tabular}

No page 2 J 


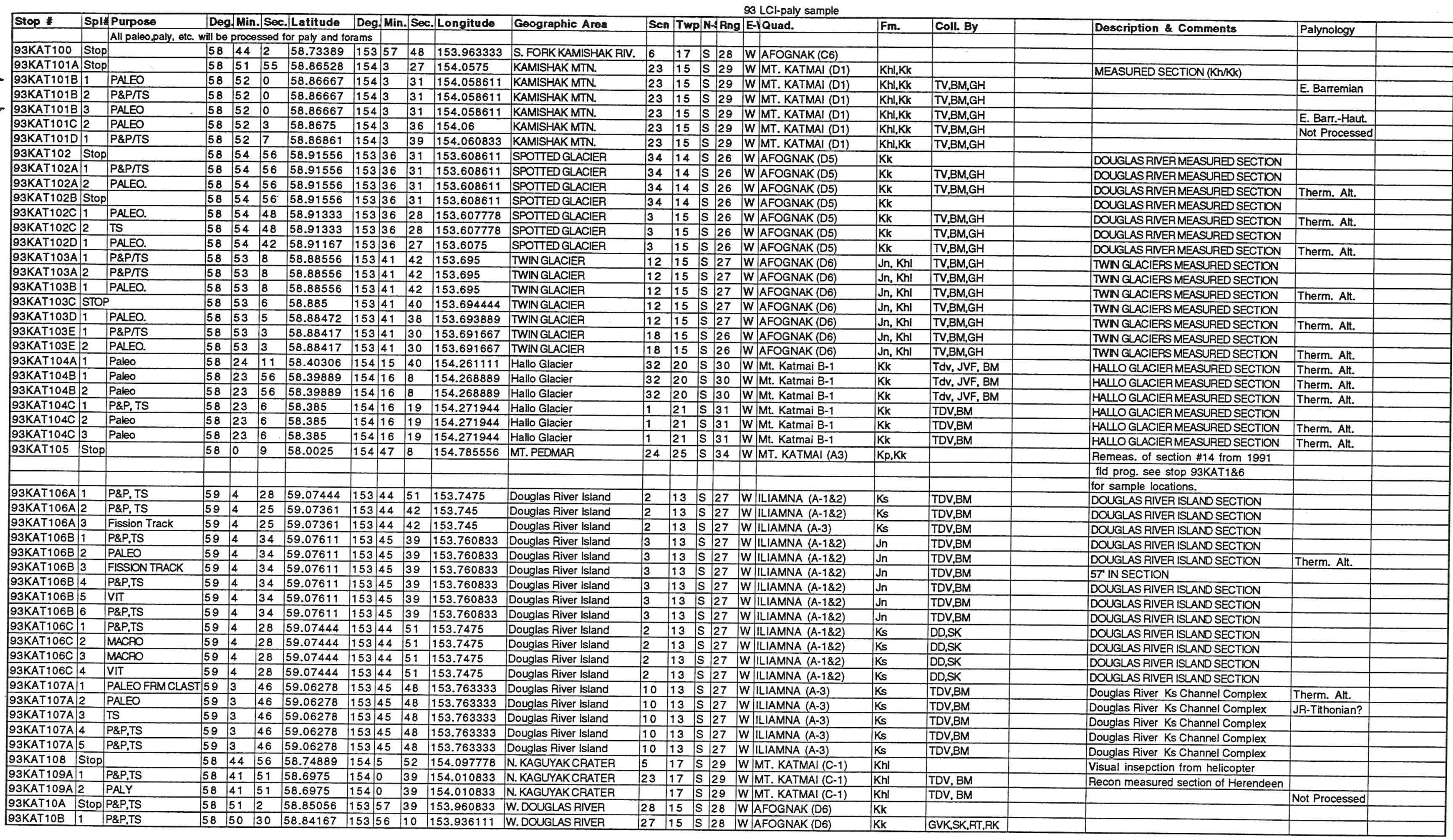




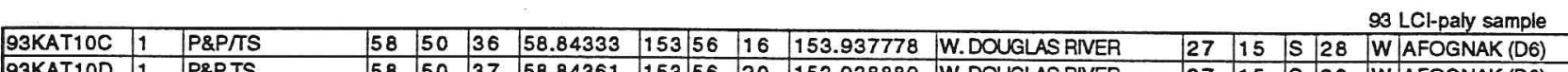

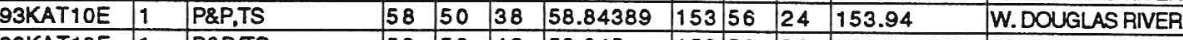

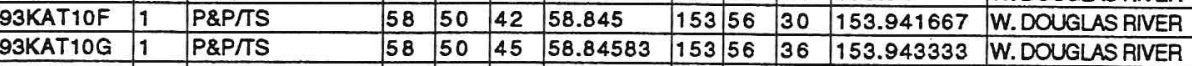

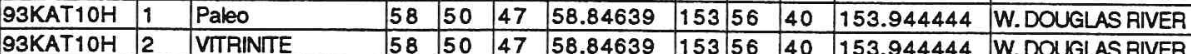

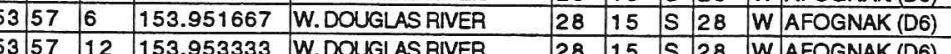

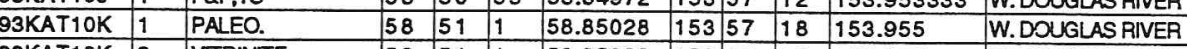
93KAT10L 11 PEP,TS 51 1 58.5528 15357 25 153.956944 W. DOUGLAS RIVER

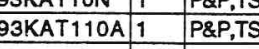

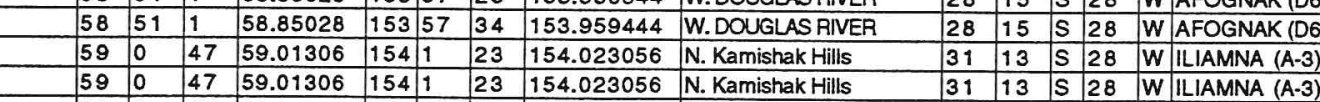
\begin{tabular}{llllll}
23 & 154.023056 & 13 & 5 & 28 & W LLAMNA A A-3) \\
\hline
\end{tabular}

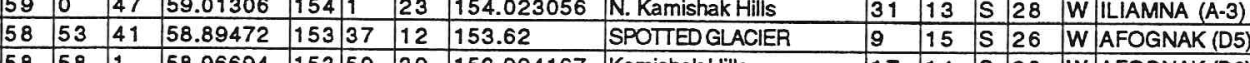

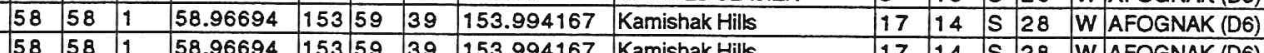
93KAT112A 3 PALEO

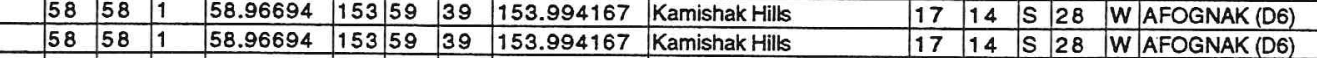

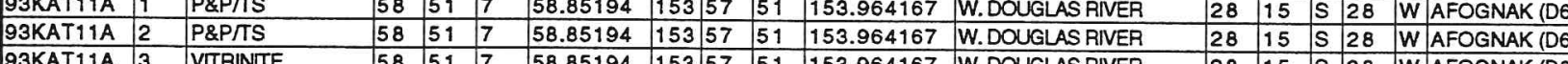

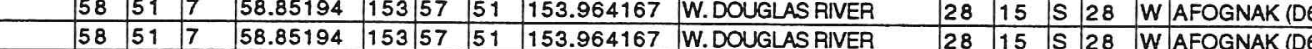

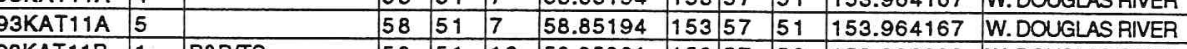

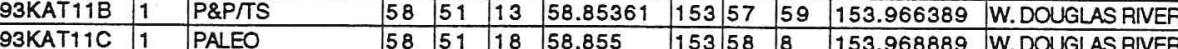

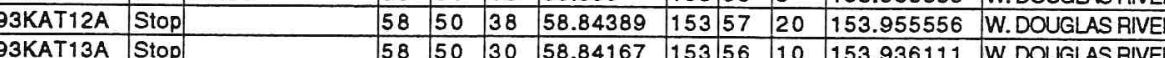

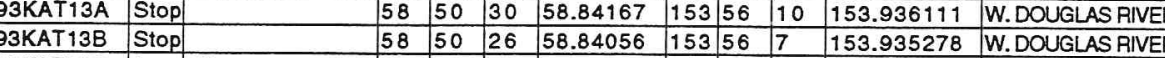
\begin{tabular}{l|l|l|l|l} 
S3KAT13C & 1 & TS \\
\hline
\end{tabular} \begin{tabular}{lll} 
93KAT13D & Stop \\
\hline 93KAT13E & 1 PALEO. \\
\hline
\end{tabular} \begin{tabular}{lllllllll}
58 & 50 & 17 & 58.83222 & 153 & 55 & 55 & 153.931944 & W. DOUGLAS RIVE \\
\hline
\end{tabular}

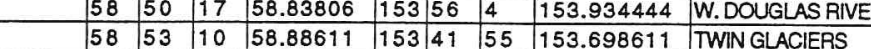

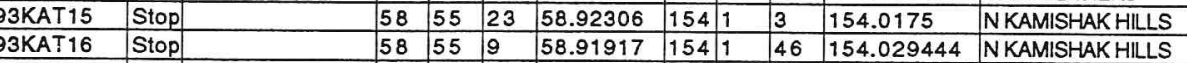

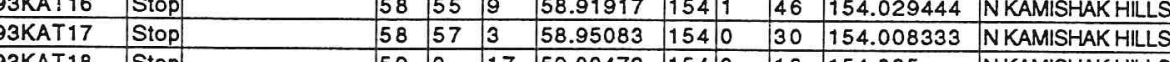

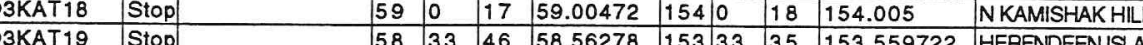

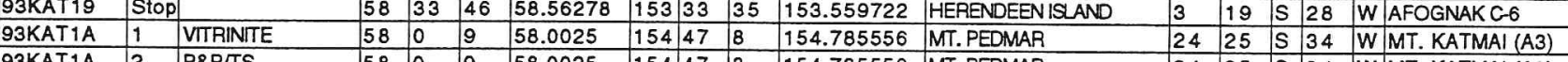

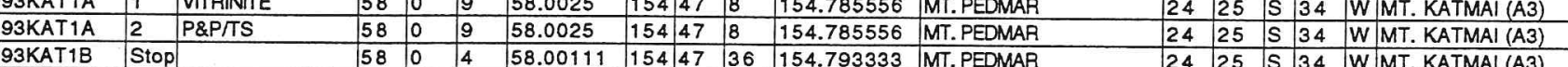

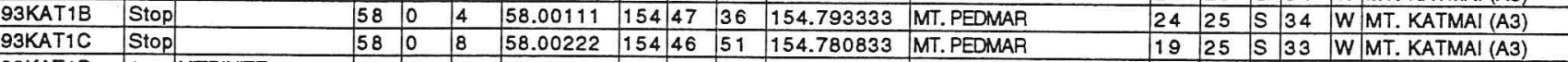

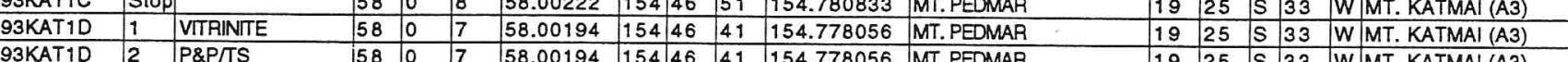

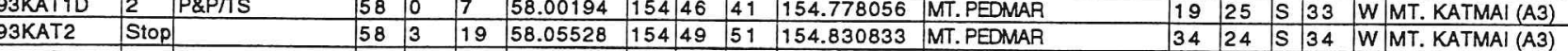

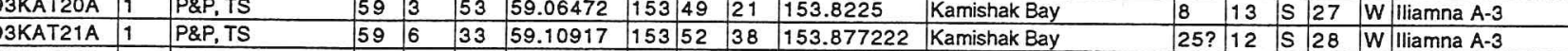

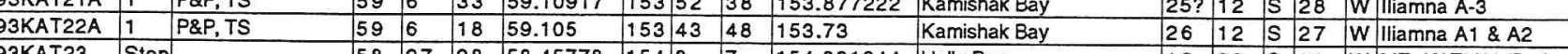

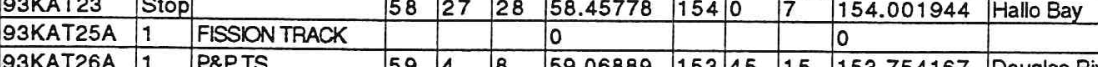
\begin{tabular}{|l|l|l|l|l|l|l|l|l|l|l|l|l|l|l|l|l}
\hline 93KAT26A & 1 & PQP,TS & 59 & 4 & 8 & 59.06889 & 153 & 45 & 15 & 153.754167 & Douglas River Island & 10 & 13 & 5 & 27 & W ILIAMNA (A-3)
\end{tabular}

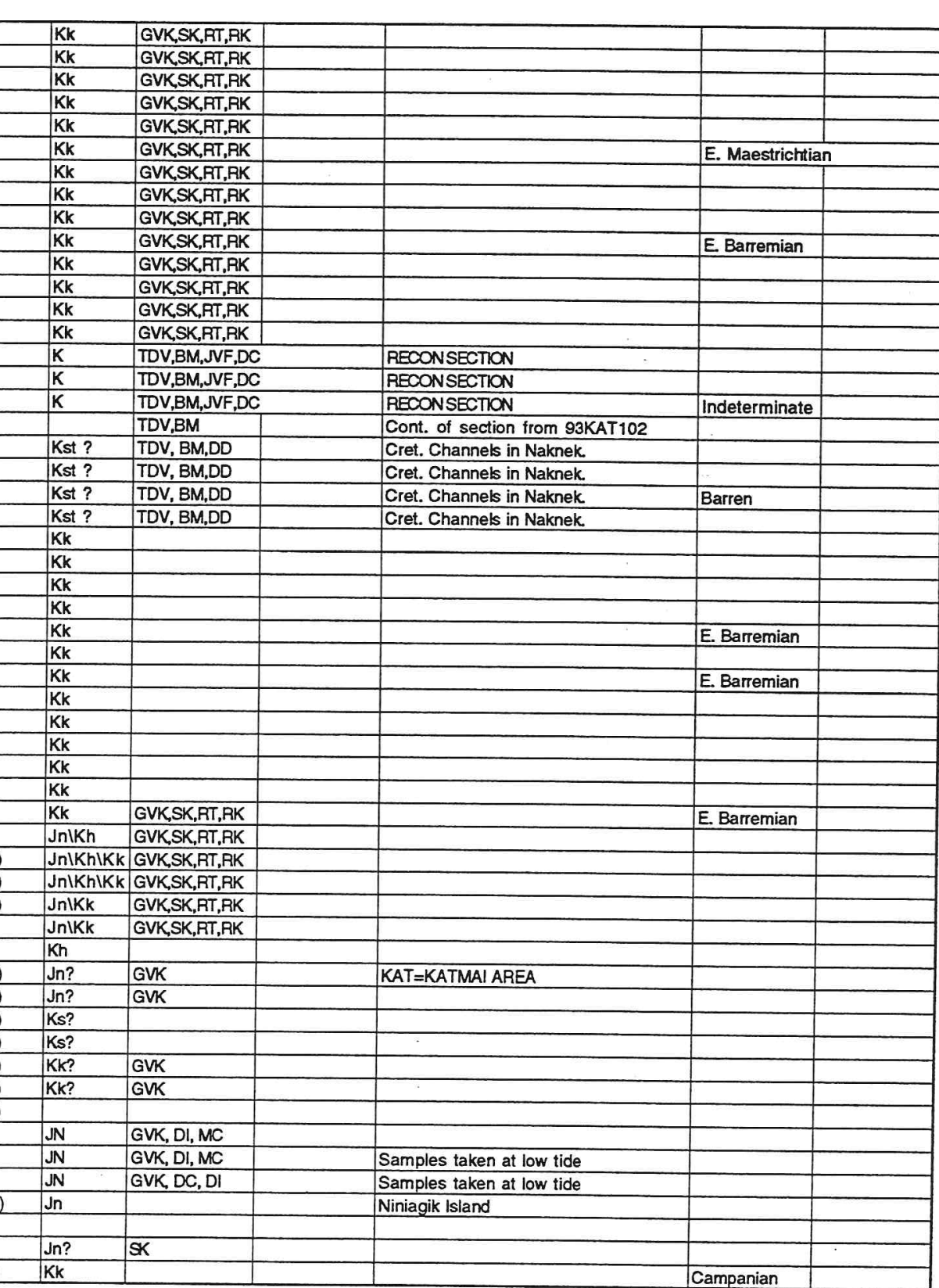




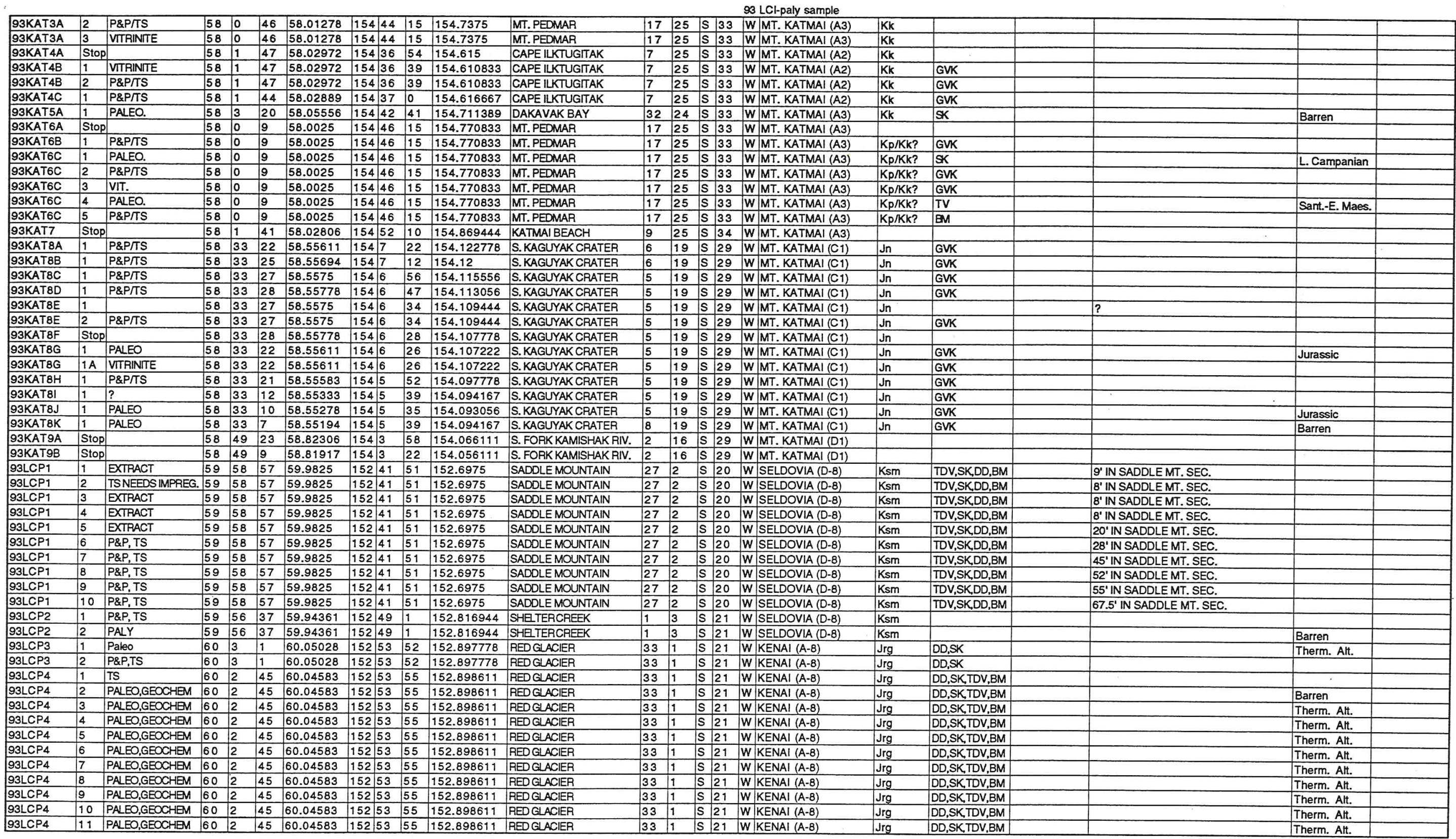




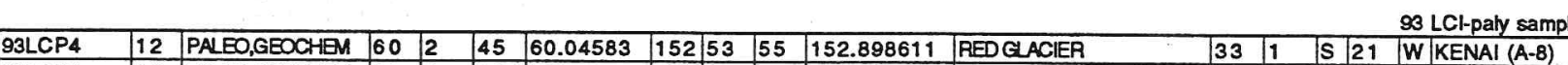

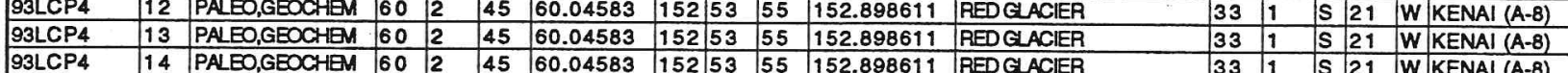

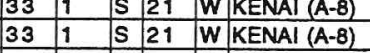

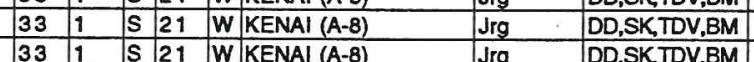

\begin{tabular}{l|l} 
Therm. Alt. \\
Therm. Att. \\
\hline
\end{tabular}

\begin{tabular}{l} 
Therm. Alt. \\
Therm. Att. \\
\hline Therm. Att.
\end{tabular}

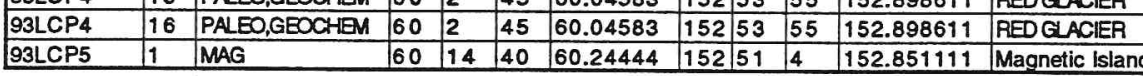

\begin{tabular}{lllll|l}
24 & 2 & N & 21 & W & KENAI \\
\hline
\end{tabular}

Therm. Alt.
Therm. Att. 


\begin{tabular}{|c|c|c|c|c|c|c|c|c|c|c|c|c|c|c|c|c|c|c|}
\hline Stop \# & Spi住 & Purpose & Deg. & Min. & Sec. & Latitude & Deg.11 & Min. & Sec. & Longitude & Geographic Area & $\operatorname{sen}$ & Twp & $N-$ Ring & E-l Quad. & Fm. & Coll. By & Description \& Comments \\
\hline & & All paleo,paly, etc. & ill be & proces & ssed fo & or paly and for & ams & & & & & & & & & & & \\
\hline 93KAT100 & Stop & & 58 & 44 & 2 & 58.73389 & 1535 & 57 & 48 & 153.963333 & S. FORK KAMISHAK RIV. & & 17 & 28 & W AFOGNAK (C6) & & & \\
\hline 93KAT101A & Stop & & 58 & 51 & 55 & 58.86528 & 1543 & & 27 & 154.0575 & KAMISHAK MTN. & 23 & 15 & 29 & W MT. KATMAI (D1) & Khl,Kk & & MEASURED SECTION (Kh/Kk) \\
\hline 93KAT101B & 1 & PALEO & 58 & 52 & 0 & 58.86667 & 154 & 3 & 31 & 154.058611 & KAMISHAK MTN. & 23 & 15 & 29 & W MT. KATMAI (D1) & Khl, Kk & TV,BM,GH & \\
\hline 93KAT101B & & P\&P/TS & 58 & 52 & 0 & 58.86667 & 154 & & 31 & 154.058611 & KAMISHAK MTN. & 23 & 15 & \begin{tabular}{l|l|}
$S$ & 29 \\
\end{tabular} & W MT. KATMAI (D1) & Khl,Kk & TV,BM,GH & \\
\hline 93KAT101B & 3 & PALEO & 58 & 52 & 0 & 58.86667 & 1545 & & 31 & 154.058611 & KAMISHAK MTN. & 23 & 15 & \begin{tabular}{l|l}
$S$ & 29 \\
\end{tabular} & W MT. KATMAI (D1) & $\mathrm{Khl}, \mathrm{Kk}$ & $\mathrm{TV}, \mathrm{BM}, \mathrm{GH}$ & \\
\hline 93KAT101C & 2 & PALEO & 58 & 52 & 3 & 58.8675 & 1543 & & 36 & 154.06 & KAMISHAK MTN. & 23 & 15 & \begin{tabular}{l|l|}
$S$ & 29 \\
\end{tabular} & W MT. KATMAI (D1) & $\mathrm{Khl}, \mathrm{Kk}$ & $\mathrm{TV}, \mathrm{BM}, \mathrm{GH}$ & \\
\hline 93KAT101D & & P\&P/TS & 58 & 52 & 7 & 58.86861 & 1545 & & 39 & 154.060833 & KAMISHAK MTN. & 23 & 15 & \begin{tabular}{l|l} 
S 29 \\
\end{tabular} & W MT. KATMAI (D1) & Khl,Kk & $\mathrm{TV}, \mathrm{BM}, \mathrm{GH}$ & \\
\hline $93 \mathrm{KAT} 102$ & Stop & & 58 & 54 & 56 & 58.91556 & 153 & 36 & 31 & 153.608611 & SPOTIEDGLACIER & 34 & 14 & \begin{tabular}{l|l|}
$S$ & 26 \\
\end{tabular} & W AFOGNAK (D5) & Kk & & DOUGLAS RIVERMEASURED SECTION \\
\hline 93KAT102A & & P\&P/TS & 58 & 54 & 56 & 58.91556 & 153 & 36 & 31 & 153.608611 & SPOTTED GLACIER & 34 & 14 & \begin{tabular}{l|l|l|l|} 
& 26 \\
\end{tabular} & W AFOGNAK (D5) & Kk & $\mathrm{TV}, \mathrm{BM}, \mathrm{GH}$ & DOUGLAS RIVER MEASURED SECTION \\
\hline 93KAT 102A & & PALEO. & 58 & 54 & 56 & 58.91556 & 153 & & 31 & 153.608611 & SPOTIEDGLACIER & 34 & 14 & \begin{tabular}{l|l|}
$S$ & 26 \\
\end{tabular} & \begin{tabular}{|l|l|} 
W & AFOGNAK (D5) \\
\end{tabular} & Kk & $\mathrm{TV}, \mathrm{BM}, \mathrm{GH}$ & DOUGLASRIVERMEASURED SECTION \\
\hline 93KAT102B & Stop & & 58 & 54 & 56 & 58.91556 & 153 & 36 & 31 & 153.608611 & SPOTTED GLACIER & 34 & 14 & 26 & W AFOGNAK (D5) & Kk & & DOUGLAS RIVER MEASURED SECTION \\
\hline 93KAT102C & 1 & PALEO. & 58 & 54 & 48 & 58.91333 & 153 & 36 & 28 & 153.607778 & SPOTTED GLACIER & 3 & 15 & \begin{tabular}{l|l}
$S$ & 26 \\
\end{tabular} & W AFOGNAK (D5) & Kk & $\mathrm{TN}, \mathrm{BM}, \mathrm{GH}$ & DOUGLAS RIVERMEASURD SECTION \\
\hline 93KAT102C & & TS & 58 & 54 & 48 & 58.91333 & 153 & 36 & 28 & 153.607778 & SPOTTED GLACIER & 3 & 15 & \begin{tabular}{l|l}
$S$ & 26 \\
\end{tabular} & W AFOGNAK (D5) & Kk & $\mathrm{TV}, \mathrm{BM}, \mathrm{GH}$ & DOUGLAS RIVER MEASURE SECTION \\
\hline 93KAT102D & & PALEO. & 58 & 54 & 42 & 58.91167 & 153 & 36 & 27 & 153.6075 & SPOTIEDGLACIER & 3 & 15 & 26 & W AFOGNAK (D5) & Kk & $\mathrm{TV}, \mathrm{BM}, \mathrm{GH}$ & DOUGLAS RIVER MEASURED SECTION \\
\hline 93КАТ 103A & & P\&PTSS & 58 & 53 & 8 & 58.88556 & 153 & 41 & 42 & 153.695 & TWIN GLACIER & 12 & 15 & 27 & W AFOGNAK (D6) & $\mathrm{Jn}, \mathrm{Khl}$ & $\mathrm{TV}, \mathrm{BM}, \mathrm{GH}$ & TWIN GLACIERS MEASURED SECTION \\
\hline 93КАТ 103A & & P\&P/TS & 58 & 53 & 8 & 58.88556 & 153 & 41 & 42 & 153.695 & TWINGLACIER & 12 & 15 & 27 & W AFOGNAK (D6) & $\mathrm{Jn}, \mathrm{Khl}$ & $T \mathrm{~T}, \mathrm{BM}, \mathrm{GH}$ & TWINGLACIERS MEASURED SECTION \\
\hline 93KAT103B & 1 & PALEO. & 58 & 53 & 8 & \begin{tabular}{|l|l|}
58.88556 \\
\end{tabular} & 153 & 41 & 42 & 153.695 & TWINGLACIER & 12 & 15 & S 27 & W AFOGNAK (D6) & $\mathrm{Jn}, \mathrm{Khl}$ & TV,BM,GH & TWIN GLACIERS MEASURED SECTION \\
\hline 93KAT103C & STOP & & 58 & 53 & 6 & 58.885 & 153 & 41 & 40 & 153.694444 & TWINGLACIER & 12 & 15 & $\begin{array}{ll}S & 27 \\
\end{array}$ & W AFOGNAK (D6) & $\mathrm{In}, \mathrm{Khl}$ & $\mathrm{TV}, \mathrm{BM}, \mathrm{GH}$ & TWIN GLACIERS MEASURED SECTION \\
\hline 93KAT103D & & PALEO. & 58 & 53 & 5 & 58.88472 & 153 & 41 & 38 & 153.693889 & TWIN GLACIER & 12 & 15 & \begin{tabular}{l|l}
$S$ & 27 \\
\end{tabular} & W AFOGNAK (D6) & $\mathrm{Jn}, \mathrm{Khl}$ & $\mathrm{TV}, \mathrm{BM}, \mathrm{GH}$ & TWIN GLACIERS MEASURED SECTION \\
\hline 93KAT103E & 1 & P\&PTTS & 58 & 53 & 3 & 58.88417 & 153 & 41 & 30 & 153.691667 & TWIN GLACIER & 18 & 15 & 26 & W AFOGNAK (D6) & $\mathrm{Jn}, \mathrm{Khl}$ & $\mathrm{TV}, \mathrm{BM}, \mathrm{GH}$ & TWINGLACIERS MEASURED SECTION \\
\hline 93KAT103E & 2 & PALEO. & 58 & 53 & 3 & 58.88417 & 153 & 41 & 30 & 153.691667 & TWIN GLACIER & 18 & 15 & \begin{tabular}{l|l|l}
$S$ & 26 \\
\end{tabular} & W AFOGNAK (D6) & $\mathrm{In}, \mathrm{Khl}$ & $\mathrm{TV}, \mathrm{BM}, \mathrm{GH}$ & TWIN GLACIERS MEASURED SECTION \\
\hline 93KA & 3 & PALEO. & 58 & 53 & 3 & 58.88417 & 153 & 41 & 30 & 153.691667 & TWIN GLACIER & 18 & 15 & \begin{tabular}{l|l}
$S$ & 26 \\
\end{tabular} & W AFOGNAK (D6) & $\mathrm{Jn}, \mathrm{Khl}$ & $\mathrm{TV}, \mathrm{BM}, \mathrm{GH}$ & TWIN GLACIERS MEASURED SECTION \\
\hline 93KAT104A & 1 & Paleo & 58 & 24 & 11 & 58.40306 & 154 & 15 & 40 & 154.261111 & Hallo Glacier & 32 & 20 & \begin{tabular}{l|l}
$S$ & 30 \\
\end{tabular} & $\begin{array}{l}\text { W } \\
\text { Mt. Katmai B-1 }\end{array}$ & Kk & $T d v, J V F, B M$ & HALLO GLACIERMEASURED SECTION \\
\hline 93KAT104B & 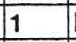 & Paleo & 58 & 23 & 56 & 58.39889 & 154 & 16 & 8 & 154.268889 & Hallo Glacier & 32 & 20 & $\begin{array}{ll}5 & 30 \\
\end{array}$ & W Mt. Katmai B-1 & $\mathrm{Kk}$ & Tdv, JVF, BM & HALLO GLACIER MEASURED SECTION \\
\hline 93KAT104B & 2 & Paleo & 58 & 23 & 56 & 58.39889 & 154 & 16 & 8 & 154.268889 & Hallo Glacier & 32 & 20 & $\begin{array}{lll}S & 30 \\
\end{array}$ & $\begin{array}{l}\text { W } \\
\text { Mt. Katmai B-1 } \\
\end{array}$ & Kk & Tdv, JVF, BM & HALLOGLACIER MEASURED SECTION \\
\hline 93KAT104C & 1 & P\&P, TS & 58 & 23 & 6 & 58.385 & 154 & 16 & 19 & 154.271944 & Hallo Glacier & 1 & 21 & \begin{tabular}{l|l|l}
$S$ & 31 \\
\end{tabular} & W Mt. Katmai B-1 & Kk & TDV,BM & HALO GLACIER MEASURED SECTION \\
\hline 93КАT 104C & 2 & Paleo & 58 & 23 & 6 & 58.385 & 154 & 16 & 19 & 154.271944 & Hallo Glacier & 1 & 21 & 31 & \begin{tabular}{|l|l|} 
W & Mt. Katmai B-1 \\
\end{tabular} & Kk & TDV,BM & HALLOGLACIER MEASURED SECTION \\
\hline 93KAT104C & 3 & Paloo & 58 & 23 & 6 & 58.385 & 154 & 16 & 19 & 154.271944 & Hallo Glacier & 1 & 21 & 31 & W Mt. Katmai B-1 & Kk & TDV,BM & HALLOGLACIERMEASURED SECTION \\
\hline 93KAT105 & Stop & & 58 & 0 & 9 & 58.0025 & 154 & 47 & 8 & 154.785556 & MT. PEDMAR & 24 & 25 & $\begin{array}{lll}5 & 34 \\
\end{array}$ & W MT. KATMAI (A3) & $\mathrm{Kp}, \mathrm{Kk}$ & & Remeas. of section \#14 from 1991 \\
\hline & & & & & & & & & & & & & & & & & & fld prog. see stop 93KAT1\&6 \\
\hline & & & & & & & & & & & & & & & & & & for sample locations. \\
\hline 93KA & 1 & P\&P, TS & 59 & 4 & 28 & 59.07444 & 153 & 44 & 51 & 153.7475 & Douglas River Island & 2 & 13 & 27 & W ILIAMNA (A-1\&2) & Ks & TDV,BM & DOUGLAS RIVER ISLAND SECTION \\
\hline 93KAT106A & 2 & P\&P, TS & 59 & 4 & 25 & 59.07361 & 153 & 44 & 42 & 153.745 & Douglas River Island & 2 & 13 & 27 & W ILIAMNA (A-1\&2) & Ks & TDV,BM & DOUGLAS RIVER ISLAND SECTION \\
\hline 93KAT106A & & Fission Track & 59 & 4 & 25 & 59.07361 & 153 & 44 & 42 & 153.745 & Douglas River Island & 2 & 13 & \begin{tabular}{l|l}
5 & 27 \\
\end{tabular} & W ILIAMNA (A-3) & Ks & TDV,BM & DOUGLAS RIVER ISLAND SECTION \\
\hline 93KAT106B & 1 & P\&P,TS & 59 & 4 & 34 & 59.07611 & 153 & 45 & 39 & 153.760833 & Douglas River Island & 3 & 13 & 27 & W ILIAMNA (A-1\&2) & $J n$ & TDV,BM & DOUGLAS RIVER ISLAND SECTION \\
\hline 93KAT106B & 2 & PALEO & 59 & 4 & 34 & 59.07611 & 153 & 45 & 39 & 153.760833 & \begin{tabular}{|l|} 
Douglas River Island \\
\end{tabular} & 3 & 13 & 27 & W ILIAMNA (A-1\&2) & In & TDV,BM & DOUGLAS RIVER ISLAND SECTION \\
\hline 93KAT 106B & 3 & FISSION TRACK & 59 & 4 & 34 & 59.07611 & 153. & 45 & 39 & 153.760833 & Douglas River Island & 3 & 13 & 27 & W ILIAMNA (A-1\&2) & $\mathrm{Jn}$ & TDV,BM & $57^{\prime}$ IN SECTION \\
\hline 93KAT 106B & 4 & P\&P,TS & 59 & 4 & 34 & 59.07611 & 153 & 45 & 39 & 153.760833 & \begin{tabular}{|l} 
Douglas River Island \\
\end{tabular} & 3 & 13 & 27 & W ILIAMNA (A-1\&2) & $\mathrm{Jn}$ & TDV,BM & DOUGLAS RIVER ISLAND SECTION \\
\hline 93KAT106B & 5 & VIT & 59 & 4 & 34 & \begin{tabular}{|l|l|}
59.07611 \\
\end{tabular} & 153 & 45 & 39 & 153.760833 & Douglas River Island & 3 & 13 & \begin{tabular}{l|l|}
$S$ & 27 \\
\end{tabular} & W ILIAMNA (A-1\&2) & In & TDV,BM & DOUGLAS RIVER ISLAND SECTION \\
\hline 93KAT106B & 6 & P\&P,TS & 59 & 4 & 34 & 59.07611 & 153 & 45 & 39 & 153.760833 & Douglas River Island & 3 & 13 & \begin{tabular}{l|l|}
$S$ & 27 \\
\end{tabular} & W ILIAMNA (A-1\&2) & $J n$ & TDV,BM & DOUGLAS RIVER ISLAND SECTION \\
\hline 93KAT106C & 1 & P\&P,TS & 59 & 4 & 28 & 59.07444 & 153 & 44 & 51 & 153.7475 & Douglas River Island & 2 & 13 & \begin{tabular}{l|l|l}
$S$ & 27 \\
\end{tabular} & W ILIAMNA (A-1\& & $K_{s}$ & $\mathrm{DD}, \mathrm{SK}$ & DOUGLAS RIVER ISLAND SECTION \\
\hline 93KAT 106C & 2 & MACAO & 59 & 4 & 28 & 59.07444 & 153. & 44 & 51 & 153.7475 & Douglas River Island & 2 & 13 & \begin{tabular}{l|l|l}
$S$ & 27 \\
\end{tabular} & W ILIAMNA (A-1\&2) & Ks & DD,SK & DOUGLAS RIVER ISLAND SECTION \\
\hline 93KAT106C & 3 & MACAO & 59 & 4 & 28 & 59.07444 & 153 & 44 & 51 & 153.7475 & \begin{tabular}{|l|} 
Douglas River Island \\
\end{tabular} & 2 & 13 & 27 & W ILIAMNA (A-1\&2) & Ks & DD,SK & DOUGLAS RIVER ISLAND SECTION \\
\hline 93KAT 106C & 4 & VIT & 59 & 4 & 28 & 59.07444 & 153 & 44 & 51 & 153. & Douglas River Island & 2 & 13 & 27 & $\begin{array}{l}\text { W } \\
\text { ILIAMNA (A-1\&2) }\end{array}$ & Ks & $\mathrm{DD}, \mathrm{SK}$ & DOUGLAS RIVER ISLAND SECTION \\
\hline 93KAT107A & & PALEO FRM CLA & 59 & 3 & 46 & 59.06278 & 153 & 45 & 48 & 153.763333 & Douglas River Island & 10 & 13 & $\begin{array}{l}27 \\
\end{array}$ & W/ILIAMNA (A-3) & Ks & TDV,BM & Douglas River Ks Channel Complex \\
\hline
\end{tabular}




\begin{tabular}{|c|c|c|c|c|c|c|c|c|c|c|c|c|c|c|c|c|c|c|c|}
\hline 93KAT107A & & PALEO & 59 & & 46 & .06278 & 153 & & 48 & 153.763333 & Douglas River Island & 10 & 13 & $5 \sqrt{2}$ & 27 & \begin{tabular}{l|l} 
W ILIAMNA (A-3) \\
\end{tabular} & Ks & TDV,BM & Douglas River Ks Channel Complex \\
\hline 93KAT107A & & TS & 59 & 3 & 46 & 59.06278 & 153 & 45 & 48 & 153.763333 & Douglas River Island & 10 & 13 & 52 & 27 & W ILIAMNA (A-3) & Ks & TDV,BM & Douglas River Ks Channel Complex \\
\hline 93KAT107A & & P\&P,TS & 59 & 3 & 46 & 59.06278 & 153 & 45 & 48 & 153.763333 & Douglas River Island & 10 & 13 & & 27 & W ILIAMNA (A-3) & Ks & TDV,BM & Douglas River Ks Channel Complex \\
\hline 93KAT107A & & P\&P,TS & 59 & 3 & 46 & 59.06278 & 153 & 45 & 48 & 153.763333 & Douglas River Island & 10 & 13 & 52 & 27 & W ILIAMNA (A-3) & Ks & TDV,BM & Douglas River Ks Channel Complex \\
\hline 93KAT108 & Stop & & 58 & 44 & 56 & 58.74889 & 1545 & & 52 & 154.097778 & N. KAGUYAKCPATER & 5 & 17 & 52 & 29 & W MT. KATMAI (C-1) & Khl & & Visual insepction from helicopter \\
\hline 93KAT109A & & \begin{tabular}{|l|} 
PRP,TS \\
PALY
\end{tabular} & 58 & \begin{tabular}{|l|}
41 \\
41
\end{tabular} & 51 & 58.6975 & $154 \mathrm{C}$ & & 39 & 154.010833 & N. KAGUYAKCRATER & 23 & 17 & 52 & $29^{\circ}$ & W MT. KATMAI (C-1) & Khl & TDV, BM & Recon measured section of Herendeen \\
\hline 93КАT109A & & PALY & 58 & 41 & 51 & 58.6975 & 154 & & 39 & 154.010833 & N. KAGUYAKCRATER & & 17 & $\mathbf{S}$ & 29 & W MT. KATMAI (C-1) & Khl & TDV, BM & \\
\hline 93KAT10A & Stop & P\&P,TS & 58 & 51 & 2 & 58.85056 & $153: 5$ & 57 & 39 & 153.960833 & W. DOUGLAS RIVER & 28 & 15 & 52 & 28 & \begin{tabular}{l|l} 
W & AFOGNAK (D6)
\end{tabular} & Kk & & \\
\hline 93KAT10B & 1 & P\&P,TS & 58 & 50 & 30 & 58.84167 & $153: 5$ & 56 & 10 & 153.936111 & W. DOUGLAS RIVER & 27 & 15 & & 28 & \begin{tabular}{l|l} 
WFOGNAK (D6) \\
\end{tabular} & Kk & GVK,SK,RT,RK & \\
\hline 93КАТ 10C & 1 & P\&P/TS & 58 & 50 & 36 & 58.84333 & 153 & 56 & 16 & 153.937778 & W. DOUGLAS RIVER & 27 & 15 & $\mathbf{S} 2$ & 28 & \begin{tabular}{l|l} 
W & AFOGNAK (D6) \\
\end{tabular} & Kk & GVK,SK,RT,RK & \\
\hline 93KAT10D & 1 & PQP,TS & 58 & 50 & 37 & 58.84361 & 1535 & 56 & 20 & 153.938889 & W. DOUGLAS RIVER & 27 & 15 & & 28 & W AFOGNAK (D6) & Kk & GVK,SK,RT,PK & \\
\hline 93KAT10E & 1 & P\&P,TS & 58 & 50 & 38 & 58.84389 & 1535 & 56 & 24 & 153.94 & W. DOUGLAS RIVER & 27 & 15 & 52 & 28 & \begin{tabular}{l|l} 
& AFOGNAK (D6)
\end{tabular} & $\mathrm{Kk}$ & GVK,SK,RT,RK & \\
\hline 93KAT10F & 1 & P\&P/TS & 58 & 50 & 42 & 58.845 & 1535 & 56 & 30 & 153.941667 & W. DOUGLAS RIVER & 27 & 15 & & 28 & \begin{tabular}{l|l} 
W & AFOGNAK (DG) \\
\end{tabular} & $\mathrm{Kk}$ & GVK,SK,RT,RK & \\
\hline 93KAT10G & 1 & P\&P/TS & 58 & 50 & 45 & 58.84583 & 1535 & 56 & 36 & 153.943333 & W. DOUGLAS RIVER & 27 & 15 & & 28 & $\begin{array}{l}\text { W AFOGNAK (D6) } \\
\end{array}$ & Kk & GVK,SK,RT,RK & \\
\hline 93KAT10H & 1 & Paleo & 58 & 50 & 47 & 58.84639 & 1535 & 56 & 40 & 153.944444 & W. DOUGLAS RIVER & 28 & 15 & 52 & 28 & \begin{tabular}{l|l} 
W & AFOGNAK (D6) \\
\end{tabular} & Kk & GVK,SK,RT,PK & \\
\hline 93KAT10H & 2 & VITRINITE & 58 & 50 & 47 & 58.84639 & 1535 & 56 & 40 & 153.944444 & W. DOUGLAS RIVER & 28 & 15 & 52 & 28 & \begin{tabular}{l|l} 
WFOGNAK (D6) \\
\end{tabular} & Kk & GVK,SK,RT,PK & \\
\hline 93KAT10I & 1 & PQP,TS & 58 & 50 & 56 & 58.84889 & 1535 & 57 & 6 & 153.951667 & W. DOUGLAS RIVER & 28 & 15 & & 28 & W AFOGNAK (D6) & Kk & GVK,SK,RT,PK & \\
\hline 93KAT10J & 1 & P\&P,TS & 58 & 50 & 59 & 58.84972 & 1535 & 57 & 12 & 153.953333 & W.DOUGLAS RIVER & 28 & 15 & $\begin{array}{ll}52 \\
\end{array}$ & 28 & W AFOGNAK (D6) & $\mathrm{Kk}$ & GVK,SK,RT,PK & \\
\hline 93KAT10K & 1 & PALEO. & 58 & 51 & 1 & 58.85028 & 1535 & 57 & 18 & 153.955 & W.DOUGLAS RIVER & 28 & 15 & & 28 & W AFOGNAK (D6) & $\mathrm{Kk}$ & GVK,SK,RT,RK & \\
\hline 93KAT10K & 2 & VITRINITE & 58 & 51 & 1 & 58.85028 & 1535 & 57 & 18 & 153.955 & W. DOUGLAS RIVER & 28 & 15 & 52 & 28 & W AFOGNAK (D6) & Kk & GVK,SK,RT,RK & \\
\hline 93KAT10L & 1 & P\&P,TS & 58 & 51 & 1 & 58.85028 & 1535 & 57 & 25 & 153.956944 & W. DOUGLAS RIVER & 28 & 15 & \begin{tabular}{l|l}
5 & 2 \\
\end{tabular} & 28 & W AFOGNAK (D6) & Kk & GVK,SK,RT,PK & \\
\hline 93КАТ1OM & 1 & P\&,P,IS & 58 & 51 & 1 & 58.85028 & 1535 & 57 & 25 & 153.956944 & W. DOUGLAS RIVER & 28 & 15 & \begin{tabular}{l|l} 
& 2 \\
\end{tabular} & 28 & W AFOGNAK (D6) & Kk & GVK,SK,RT,RK & \\
\hline 93KAT1ON & 1 & P\&P,TS & 58 & 51 & 1 & 58.85028 & 1535 & 57 & 34 & 153.959444 & W. DOUGLAS RIVER & 28 & 15 & 52 & 28 & W AFOGNAK (D6) & $\mathrm{Kk}$ & GVK,SK,RT,RK & \\
\hline 93KAT110A & & P\&P,TS & 59 & 0 & 47 & 59.01306 & 1541 & & 23 & 154.023056 & N. Kamishak Hills & 31 & 13 & 52 & 28 & W ILIAMNA (A-3) & $\mathrm{K}$ & TDV,BM,JVF,DC & RECONSECTION \\
\hline 93KAT110A & & TS & 59 & 0 & 47 & 59.01306 & 1541 & & 23 & 154.023056 & N. Kamishak Hills & 31 & 13 & \begin{tabular}{l|l}
5 & 2 \\
\end{tabular} & 28 & W ILIAMNA (A-3) & $K$ & TDV,BM,JVF,DC & RECONSECTION \\
\hline 93KAT110A & & $\begin{array}{l}\text { PALEO } \\
\text { TS }\end{array}$ & 59 & 0 & 47 & 59.01306 & 1541 & & 23 & 154.023056 & N. Kamishak Hills & 31 & 13 & & 28 & W ILIAMNA $(A-3)$ & $K$ & TDV,BM,JVF,DC & RECONSECTION \\
\hline 93KAT111A & 1 & TS & 58 & 53 & 41 & 58.89472 & 1533 & 37 & 12 & 153.62 & SPOTTED GLACIER & 9 & 15 & \begin{tabular}{l|l}
5 & 2 \\
\end{tabular} & 26 & W AFOGNAK (D5) & & TDV,BM & Cont. of section from 93KAT102 \\
\hline 93КАT112A & & P\&P/TS & 58 & 58 & 1 & 58.96694 & 1535 & 59 & 39 & 153.994167 & Kamishak Hills & 17 & 14 & $\mathrm{~s} 2$ & 28 & \begin{tabular}{l|l} 
W & AFOGNAK (D6) \\
\end{tabular} & Kst? & TDV, BM,DD & Cret. Channels in Naknek. \\
\hline 93KAT112A & & MACAO & 58 & 58 & 1 & 58.96694 & 1535 & 59 & 39 & 153.994167 & Kamishak Hills & 17 & 14 & $\begin{array}{lll}S & 2 \\
S\end{array}$ & 28 & $\begin{array}{l}\text { W } \\
\text { AFOGNAK (D6) }\end{array}$ & Kst? & TDV, BM,DD & Cret. Channels in Naknek. \\
\hline 93KAT112A & & PALEO & 58 & 58 & 1 & 58.96694 & 1535 & 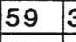 & 39 & 153.994167 & Kamishak Hills & 17 & 14 & 52 & 28 & \begin{tabular}{l|l} 
W & AFOGNAK (D6) \\
\end{tabular} & Kst? & TDV, BM,DD & Cret. Channels in Naknek. \\
\hline 93KAT112A & 4 & TS & 58 & 58 & 1 & 58.96694 & 1535 & 59 & 39 & 153.994167 & Kamishak Hills & 17 & 14 & 2 & 28 & $\begin{array}{l}\text { W } \\
\text { AFOGNAK (D6) } \\
\end{array}$ & Kst? & TDV, BM,DD & Cret. Channels in Naknek. \\
\hline 93KAT11A & 1 & P\&P/TS & 58 & 51 & 7 & 58.85194 & 1535 & 57 & 51 & 153.964167 & W. DOUGLAS RIVER & 28 & 15 & 52 & 28 & W AFOGNAK (D6) & Kk & & \\
\hline 93KAT11A & 2 & P\&P/TS & 58 & 51 & 7 & 58.85194 & 1535 & 57 & 51 & 153.964167 & W. DOUGLAS RIVER & 28 & 15 & $S 2$ & 28 & \begin{tabular}{l|l|} 
AFOGNAK (D6) \\
\end{tabular} & Kk & & \\
\hline 93KAT11A & 3 & VITRINITE & 58 & 51 & 7 & 58.85194 & 1535 & 57 & 51 & 153.964167 & W. DOUGLAS RIVER & 28 & 15 & \begin{tabular}{|l|l}
5 & 2 \\
\end{tabular} & & \begin{tabular}{|l|l|} 
AFOGNAK (D6) \\
\end{tabular} & $\mathrm{Kk}$ & & \\
\hline 93KAT11A & 4 & & 58 & 51 & 7 & 58.85194 & 1535 & 57 & 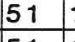 & 153.964167 & W. DOUGLAS RIVER & 28 & 15 & & 28 & W AFOGNAK (D6) & Kk & & \\
\hline 93KAT11A & 5 & & 58 & 51 & 7 & 58.85194 & 1535 & \begin{tabular}{l|l}
57 & 5 \\
\end{tabular} & 51 & 153.964167 & W. DOUGLAS RIVER & 28 & 15 & \begin{tabular}{l|l} 
& 2 \\
\end{tabular} & 88 & $\begin{array}{l}\text { W AFOGNAK (D6) } \\
\end{array}$ & Kk & & \\
\hline 93KAT11B & 1 & P\&PTS & 58 & 51 & 13 & 58.85361 & 1535 & \begin{tabular}{l|l}
57 & 5 \\
\end{tabular} & \begin{tabular}{|l|l}
59 & 1 \\
\end{tabular} & 153.966389 & W. DOUGLAS RIVER & 28 & 15 & $\begin{array}{l}S 2 \\
\end{array}$ & 28 & N AFOGNAK (D6) & Kk & & \\
\hline 93KAT11C & 1 & PALEO & 58 & 51 & 18 & 58.855 & 1535 & $\begin{array}{lll}58 \quad 8 \\
\end{array}$ & H & 153.968889 & W. DOUGLAS RIVER & 28 & 15 & & 8 & N AFOGNAK (D6) & $\mathrm{Kk}$ & & \\
\hline 93КАТ 12A & Stop & & 58 & 50 & 38 & 58.84389 & 1535 & \begin{tabular}{l|l}
$57 \quad 2$ \\
\end{tabular} & 20 & 153.955556 & W. DOUGLAS RIVER & 28 & 15 & 52 & 8 & N AFOGNAK (D6) & Kk & & \\
\hline 93KAT13A & Stop & & 58 & 50 & 30 & 58.84167 & 1535 & \begin{tabular}{l|l}
56 & 1 \\
\end{tabular} & \begin{tabular}{|l|l|}
0 & 1 \\
\end{tabular} & 153.936111 & W. DOUGLAS RIVER & 27 & 15 & $\begin{array}{ll}52 \\
\end{array}$ & & N AFOGNAK (DG) & $\mathrm{Kk}$ & & \\
\hline 93KAT13B & Stop & & 58 & 50 & 26 & 58.84056 & 1535 & \begin{tabular}{l|l}
56 & 7 \\
\end{tabular} & 7 & 153.935278 & W. DOUGLAS RIVER & 34 & 15 & & 8 & & Kk & & \\
\hline 93KAT13C & 5 & TS & 58 & 50 & 11 & 58.83639 & 1535 & \begin{tabular}{l|l}
56 & 1 \\
\end{tabular} & 7 & 153.933611 & W. DOUGLAS RIVER & 34 & 15 & $\begin{array}{ll}5 & 2 \\
\end{array}$ & 8 & $N$ AFOGNAK (DG) & $\mathrm{Kk}$ & & \\
\hline 93KAT13D & Stop & & 58 & 49 & 56 & 58.83222 & \begin{tabular}{l|l|l}
153 & 5 \\
\end{tabular} & \begin{tabular}{l|l}
55 & 5 \\
\end{tabular} & 55 & 153.931944 & W. DOUGLAS RIVER & 34 & 15 & $\mathrm{~S} 22$ & 8 & \begin{tabular}{l|l}
$N$ AFOGNAK (DG) \\
\end{tabular} & Kk & & \\
\hline 93KAT13E & 1 & PALEO. & 58 & 50 & 17 & 58.83806 & \begin{tabular}{ll|l}
153 & 5 \\
\end{tabular} & $\begin{array}{ll}56 \quad 4 \\
\end{array}$ & H & 153.934444 & W. DOUGLAS RIVER & 34 & 15 & $\begin{array}{ll}52 \\
\end{array}$ & & \begin{tabular}{l|l}
$N$ AFOGNAK (D6) \\
\end{tabular} & Kk & GVK,SK,RT,RK & \\
\hline 93KAT14 & Stop & & 58 & 53 & 10 & 58.88611 & \begin{tabular}{r|r}
1534 \\
\end{tabular} & \begin{tabular}{l|l}
41 & 5 \\
\end{tabular} & \begin{tabular}{l|l}
55 & 1 \\
\end{tabular} & 153.698611 & TWINGLACIERS & 12 & 15 & 2 & 7 & \begin{tabular}{l|l}
$N$ AFOGNAK (DG) \\
\end{tabular} & JnIKh & GVK,SK,RT,PK & \\
\hline KAT15 & Stop & & 58 & 55 & 23 & 58.92306 & \begin{tabular}{l|l}
154 & 1 \\
\end{tabular} & 1 & 3 & 154.0175 & N KAMISHAK HILS & 31 & 14 & 2 & 8 & \begin{tabular}{|l|l|} 
MT. KATMAI (D1
\end{tabular} & JnIKh| & GVK,SK,RT,RK & \\
\hline KAT16 & Stop & & 58 & 55 & 9 & 58.91917 & \begin{tabular}{|l|l|}
154 & 1 \\
\end{tabular} & & 46 & 154.029444 & VKAMISHAK HILLS & 36 & 14 & 2 & & 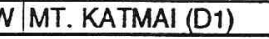 & JnIKh & GVK,SK,RT,RK & \\
\hline
\end{tabular}




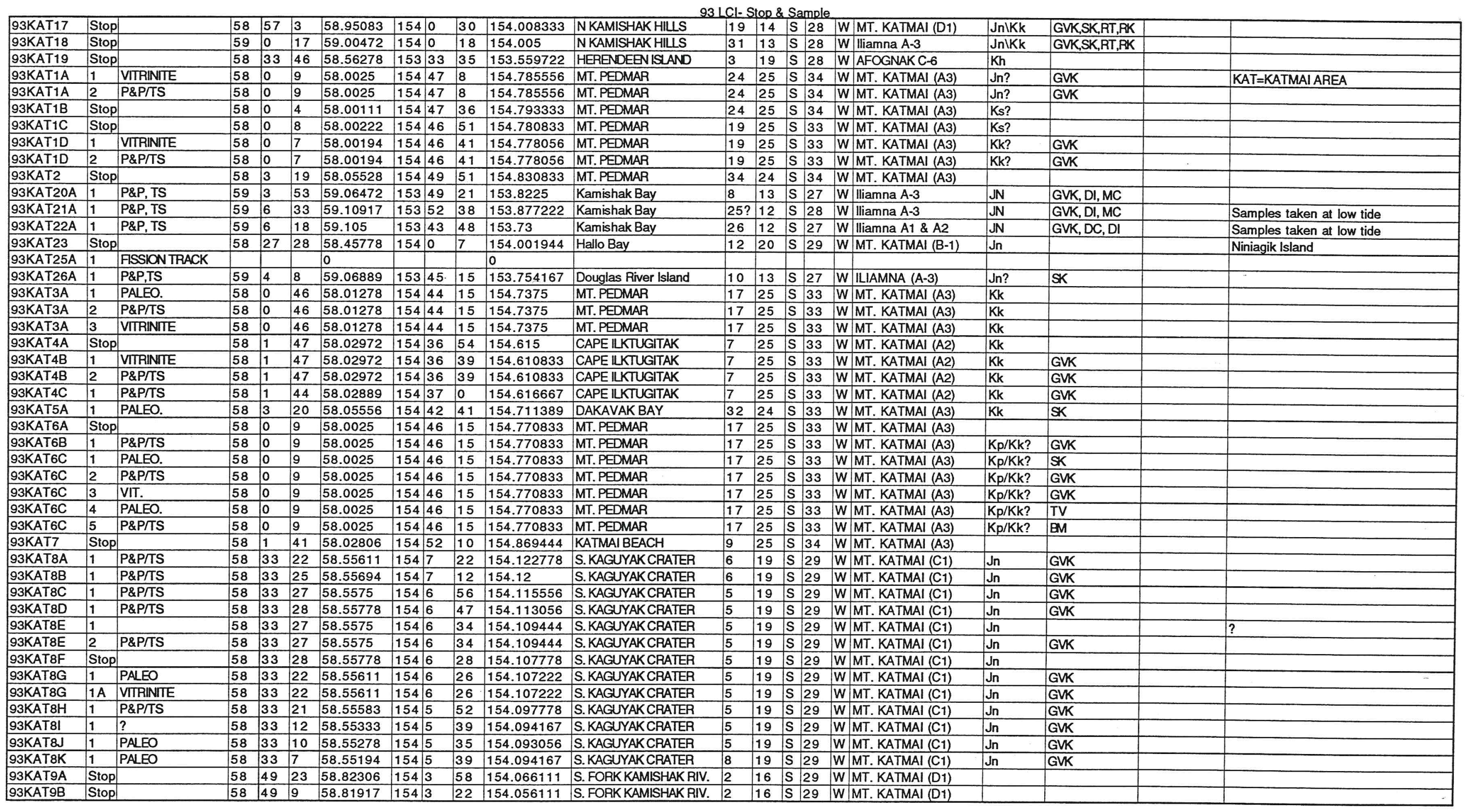

Page 3 


\begin{tabular}{|c|c|c|c|c|c|c|c|c|c|c|c|c|c|c|c|c|c|c|c|}
\hline $93 \mathrm{LCP} 1$ & 1 & EXTRACT & 59 & 58 & 57 & 59.9825 & 1524 & & 51 & 152.6975 & SADDLE MOUNTAIN & 27 & 2 & & 20 & \begin{tabular}{l|l} 
WELDOVIA (D-8) \\
\end{tabular} & Ksm & TDV,SK,DD,BM & 9' IN SADDLE MT. SEC. \\
\hline 93LCP1 & 2 & TS NEEDS IMPREG & 59 & 58 & 57 & 59.9825 & 1524 & 41 & 51 & 152.6975 & SADDLE MOUNTAIN & 27 & 2 & & 20 & W SELDOVIA (D-8) & Ksm & TDV,SK,DD,BM & 8' IN SADDLE MT. SEC. \\
\hline 93LCP1 & 3 & EXIRACT & 59 & 58 & 57 & 59.9825 & 1524 & 41 & 51 & 152.6975 & SADDLE MOUNTAIN & 27 & 2 & s: & 20 & W SELDOVIA (D-8) & $\mathrm{Ksm}$ & TDV,SK,DD,BM & $\begin{array}{l}\text { ' IN SADDLE MT. SEC. } \\
\end{array}$ \\
\hline $93 \mathrm{LCP} 1$ & 4 & EXIRACT & 59 & 58 & 57 & 59.9825 & $152 \mid 4$ & 41 & 51 & 152.6975 & SADDLE MOUNTAIN & 27 & 2 & s: & 20 & W SELDOVIA (D-8) & Ksm & TDV,SK,DD,BM & $8^{\prime}$ IN SADDLE MT. SEC. \\
\hline 93LCP1 & 5 & EXTRACT & 59 & 58 & 57 & 59.9825 & 1524 & 41 & 51 & 152.6975 & SADDLE MOUNTAIN & 27 & 2 & s: & 20 & $\begin{array}{l}\text { W SELDOVIA (D-8) } \\
\end{array}$ & Ksm & TDV,SK,DD,BM & $20^{\circ}$ IN SADDLE MT. SEC. \\
\hline 93LCP1 & 6 & P\&P, TS & 59 & 58 & 57 & 59.9825 & 1524 & 41 & 51 & 152.6975 & SADDLE MOUNTAIN & 27 & 2 & $s:$ & 20 & W SELDOVIA (D-8) & Ksm & TDV,SK,DD,BM & $28^{\prime}$ IN SADDLE MT. SEC. \\
\hline $93 \mathrm{LCP} 1$ & 7 & P\&P, TS & 59 & 58 & 57 & 59.9825 & 1524 & 41 & 51 & 152.6975 & SADDLE MOUNTAIN & 27 & 2 & S: & 20 & W SELDOVIA (D-8) & Ksm & TDV,SK,DD,BM & 45' IN SADDLE MT. SEC. \\
\hline 93LCP1 & 8 & P\&P, TS & 59 & 58 & 57 & 59.9825 & 1524 & 41 & 51 & 152.6975 & SADDLE MOUNTAIN & 27 & 2 & S & 20 & \begin{tabular}{l|l} 
W SELDOVIA (D-8) \\
\end{tabular} & Ksm & TDV,SK,DD,BM & $52^{\prime}$ IN SADDLE MT. SEC. \\
\hline 93LCP1 & 9 & P\&P, TS & 59 & 58 & 57 & 59.9825 & 152 & 41 & 51 & 152.6975 & SADDLEMOUNTAIN & 27 & 2 & S: & 20 & W SELDOVIA (D-8) & Ksm & TDV,SK,DD,BM & 55' IN SADDLE MT. SEC. \\
\hline 93LCP1 & 10 & P\&P, TS & 59 & 58 & 57 & 59.9825 & 152 & 41 & 51 & 152.6975 & SADDLE MOUNTAIN & 27 & 2 & & 20 & W SELDOVIA (D-8) & Ksm & TDV,SK,DD,BM & 67.5' IN SADDLEMT. SEC. \\
\hline $93 \mathrm{LCP2}$ & 1 & P\&P, TS & 59 & 56 & 37 & 59.94361 & 152 & 49 & 1 & 152.816944 & SHELTERCREEK & 1 & 3 & S: & 21 & $\begin{array}{l}\text { W SELDOVIA (D-8) } \\
\end{array}$ & Ksm & & \\
\hline $93 L C P 2$ & 2 & PALY & 59 & 56 & 37 & 59.94361 & 152 & 49 & 1 & 152.816944 & SHELTERCREAK & 1 & 3 & s & 21 & W SELDOVIA (D-8) & Ksm & & \\
\hline 93LCP3 & 1 & Paleo & 60 & 3 & 1 & 60.05028 & 1525 & 53 & 52 & 152.897778 & REDGLACIER & 33 & 1 & s: & 21 & W $\operatorname{KENAI}(A-8)$ & $\mathrm{Jrg}$ & $\mathrm{DD}, \mathrm{SK}$ & \\
\hline $93 L C P 3$ & 2 & P\&P,TS & 60 & 3 & 1 & 60.05028 & 1525 & 53 & 52 & 152.897778 & REDGLACIER & 33 & 1 & s & 21 & W $\operatorname{KENAI}(A-8)$ & $\mathrm{Jrg}$ & $\mathrm{DD}, \mathrm{SK}$ & \\
\hline 93LCP4 & 1 & TS & 60 & 2 & 45 & 60.04583 & 1525 & 53 & 55 & 152.898611 & REDGLACIER & 33 & 1 & & 21 & W KENAI $(A-8)$ & $\mathrm{Jrg}$ & $\mathrm{DD}, \mathrm{SK}, \mathrm{TDV}, \mathrm{BM}$ & \\
\hline $93 \mathrm{LCP} 4$ & 2 & PALEO,GEOCHEM & 60 & 2 & 45 & 60.04583 & 1525 & 53 & 55 & 152.898611 & REDGLACIER & 33 & 1 & s: & 21 & W $\operatorname{KENAI}(A-8)$ & $\operatorname{Jrg}$ & DD,SK,IDV,BM & \\
\hline $93 \mathrm{LCP} 4$ & 3 & PALEO,GEOCHEM & 60 & 2 & 45 & 60.04583 & 1525 & 53 & 55 & 152.898611 & REDGLACIER & 33 & 1 & s & 21 & W $\operatorname{KENAI~}(A-8)$ & Jrg & $\mathrm{DD}, \mathrm{SK}, \mathrm{TDV}, \mathrm{BM}$ & \\
\hline $93 \mathrm{LCP} 4$ & 4 & PALEO,GEOCHEM & 60 & 2 & 45 & 60.04583 & 1525 & 53 & 55 & 152.898611 & RED GLACIER & 33 & 1 & & 21 & W $\operatorname{KENAI~}(A-8)$ & $\mathrm{Jrg}$ & $\mathrm{DD}, \mathrm{SK}, \mathrm{TDV}, \mathrm{BM}$ & \\
\hline $93 L C P 4$ & 5 & PALEO,GEOCHEM & 60 & 2 & 45 & 60.04583 & 1525 & 53 & 55 & 152.898611 & REDGLACIER & 33 & 1 & $s$ & 21 & W $\operatorname{KENAI}(A-8)$ & $\mathrm{Jrg}$ & $\mathrm{DD}, \mathrm{SK}, \mathrm{TDV}, \mathrm{BM}$ & \\
\hline $93 L C P 4$ & 6 & PALEO,GEOCHEM & 60 & 2 & 45 & 60.04583 & 1525 & 53 & 55 & 152.898611 & REDGLACIER & 33 & 1 & & 21 & W $\mid \operatorname{KENAI}(A-8)$ & Jrg & $\mathrm{DD}, \mathrm{SK}, \mathrm{TDV}, \mathrm{BM}$ & \\
\hline 93 LCP4 & 7 & PALEO,GEOCHEM & 60 & 2 & 45 & 60.04583 & 1525 & & 55 & 152.898611 & RED GLACIER & 33 & 1 & s & 21 & $\begin{array}{l}\mathrm{W} \\
\mathrm{KENAI}(\mathrm{A}-8)\end{array}$ & $\mathrm{Jrg}$ & $\mathrm{DD}, \mathrm{SK}, \mathrm{DDV}, \mathrm{BM}$ & \\
\hline 93LCP4 & 8 & PALEO,GEOCHEM & 60 & 2 & 45 & 60.04583 & 1525 & 53 & 55 & 152.898611 & REDGLACIER & 33 & 1 & $s$ & 21 & W $\operatorname{KENAI}(A-8)$ & $\mathrm{Jrg}$ & DD,SK,TDV,BM & \\
\hline 93LCP4 & 9 & PALEO,GEOCHEM & 60 & 2 & 45 & 60.04583 & 1525 & 53 & 55 & 152.898611 & PED GLACIER & 33 & 1 & & 21 & $\begin{array}{l}\text { W } \\
\operatorname{KENAI}(A-8)\end{array}$ & $\mathrm{Jrg}$ & $\mathrm{DD}, \mathrm{SK}, \mathrm{TDV}, \mathrm{BM}$ & \\
\hline 93LCP4 & 10 & PALEO,GEOCHEM & 60 & 2 & 45 & 60.04583 & 1525 & & 55 & 152.898611 & RED GLACIER & 33 & 1 & $\begin{array}{ll}s & 2 \\
\end{array}$ & 21 & W $\operatorname{KENAI}(A-8)$ & $\mathrm{Jrg}$ & $\mathrm{DD}, \mathrm{SK}, \mathrm{TDV}, \mathrm{BM}$ & \\
\hline $93 \mathrm{LCP} 4$ & 11 & PALEO,GEOCHEM & 60 & 2 & 45 & 60.04583 & 152 & 53 & 55 & 152.898611 & RED GLACIER & 33 & 1 & $s$ & 21 & W $\operatorname{KENAI}(A-8)$ & $\mathrm{Jrg}$ & $\mathrm{DD}, \mathrm{SK}, \mathrm{TDV}, \mathrm{BM}$ & \\
\hline 93LCP4 & 12 & PALEO,GEOCHEM & 60 & 2 & 45 & 60.04583 & $152:$ & 53 & 55 & 152.898611 & REDGLACIEA & 33 & 1 & s: & 1 & W $\operatorname{KENAI}(A-8)$ & $\operatorname{srg}$ & DD,SK, TDV,BM & \\
\hline 93LCP4 & 13 & PALEO,GEOCHEM & 60 & 2 & 45 & 60.04583 & 152 & & 55 & 152.898611 & REDGLACIER & 33 & 1 & \begin{tabular}{l|l}
$S 2$ \\
\end{tabular} & 1 & W $\operatorname{KENAI}(A-8)$ & $\mathrm{Jrg}$ & $\mathrm{DD}, \mathrm{SK}, \mathrm{TDV}, \mathrm{BM}$ & \\
\hline 93LCP4 & 14 & PALEO,GEOCHEM & 60 & 2 & 45 & 60.04583 & 152 & 53 & 55 & 152.898611 & RED GLACIER & 33 & 1 & $s 2$ & 21 & \begin{tabular}{l|l} 
W & $\operatorname{KENAI}(A-8)$ \\
\end{tabular} & $\mathrm{Jrg}$ & $\mathrm{DD}, \mathrm{SK}, \mathrm{TDV}, \mathrm{BM}$ & \\
\hline $93 L C P 4$ & 15 & PALEO,GEOCHEM & 60 & 2 & 45 & 60.04583 & $152:$ & 53 & 55 & 152.898611 & RED GLACIER & 33 & 1 & & 1 & W $\operatorname{KENAI}(A-8)$ & $\mathrm{Jrg}$ & $\mathrm{DD}, \mathrm{SK}, \mathrm{TDV}, \mathrm{BM}$ & \\
\hline 93LCP4 & 16 & PALEO,GEOCHEM & 60 & 2 & 45 & 60.04583 & $152:$ & 53 & 55 & 152.898611 & REDGLACIER & 33 & 1 & $\begin{array}{ll}5 & 2 \\
\end{array}$ & 1 & W $\mid \operatorname{KENAI}(A-8)$ & Jrg & DD,SK,IDV,BM & \\
\hline 93 LCP5 & 1 & MAG & 60 & 14 & 40 & 60.24444 & 152 & 51 & 4 & 152.851111 & Magnetic Island & 24 & & & & W $\mid \operatorname{KENAI}(\mathrm{A}-8)$ & & & \\
\hline
\end{tabular}




\section{GEOCHRON LABORATORIES a division of KRUEGER ENTERPRISES, INC.}

711 CONCORD AVENUE + CAMBRIDGE, MASSACHUSETTS O2138 + U. $\$, A$ TELEPHONE: (617) 876.3691 TELEFAX: $(617) 661.0148$

Our Sample No. B-10447

Your Reierence: Phonecal1 of $12 / 3 / 93$

Submitted By: Steven C. Bergman

ARCO Exploration and Production Technology

2300 West Plano Parkway

Plano, TX 75075

Sample Description \& Locality: Sample \# $93 \mathrm{KAT} 25 \mathrm{~A}-1$, volcanic sandstone
Date Received: $9 / 22 / 93$

Date Reported: $1 / 6 / 94$

Material Analyzed: Biotite concentrate, $-80 /+200$ mesh.

${ }^{40} \mathrm{Ar} / 40 \mathrm{~K}=\quad .008984 \quad$ AGE $=148+1=4$ M.Y.

Argon Analyses:

$\begin{array}{ccc}{ }^{40} \mathrm{Ar}, \mathrm{ppm} & { }^{\circ 0} \text { Ar/Total }{ }^{\circ} \mathrm{Ar} & \text { Ave. }{ }^{40} \text { Ar, ppm } \\ .01899 & .358 & .01890 \\ .01880 & .576 & \end{array}$

Potassium Analyses:

$\begin{array}{lcr}\% K & \text { Ave. } \% K & \text { 40K, ppm } \\ 1.788 & 1.763 & 2.103 \\ 1.738 & & \end{array}$

Constants Used:

$\lambda_{b}=4.962 \times 10.10 / \mathrm{year}$

$\left(\lambda_{0}+\lambda_{0}^{\prime}\right)=0.581 \times 10.10 /$ year

${ }^{40} \mathrm{~K} / \mathrm{K}=1.193 \times 10^{-4} \mathrm{~g} / \mathrm{g}$

$A G E=\frac{1}{\lambda_{p}+\left(\lambda_{0}+\lambda_{0}^{\prime}\right)} \ln \left[\frac{\lambda_{0}+\left(\lambda_{0}+\lambda_{0}^{\prime}\right)}{\left(\lambda_{0}+\lambda_{0}^{\prime}\right)} \times \frac{40 \cdot A r}{40 K}+1\right]$

Note: ${ }^{\circ}$ Ar refers to radiogenic ${ }^{40} \mathrm{Ar}$.

M.Y. refers to millions of years. 


\section{GEOCHRON LABORATORIES a division of}

KRUEGER ENTERPRISES, INC.

711 CONCORO AVENUE + CAMBRIDGE. MASSACHUSETTS O2138 * U.S.A

Our Sample No. $A-10447$

Date Recelved: $\quad 9 / 22 / 93$

Your Reference: Phonecall of $11 / 18 / 93$

Date Reported: $1 / 6 / 94$

Submitted By: Steven C. Bergman

ARCO Exploration and Production Technology

2300 West Plano Parkway

Plano, TX 75075

Sample Description \& Locality: Sample "\# $93 \mathrm{KAT} 25 \mathrm{~A}-1$ voloanic gandstone

Materlal Analyzed:

Amphibole concentrate, $-80 /+200$ mesh.

$\omega^{\circ \cdot} \mathrm{Ar} /{ }^{100} \mathrm{~K}=$

.01047

AGE $=171+/=6 \mathrm{M} . \mathrm{Y}$.

Argon Analyses:

$\begin{array}{ccc}{ }^{40} \text { Ar, ppm } & { }^{\circ 0} \text { Ar } / \text { Total }{ }^{\circ 0} \mathrm{Ar} & \text { Ave. }{ }^{40} \mathrm{Ar}, \mathrm{ppm} \\ .004635 & .556 & .004588 \\ .004549 & .525 & \end{array}$

Potassium Analyses:
$\% \mathrm{~K}$
0.369
Ave. $\% \mathrm{~K}$
4k, ppm
0.370
0.370
0.441

Constants Used:

$\lambda_{b}=4.962 \times 10^{-10} /$ year

$\left(\lambda_{c}+\lambda_{0}^{\prime}\right)=0.581 \times 10.10 /$ year

${ }^{40 K} \mathrm{~K} K=1.193 \times 10.4 \mathrm{~g} / \mathrm{g}$

$A G E=\frac{1}{\lambda_{s}+\left(\lambda_{0}+\lambda_{0}^{\prime}\right)} \ln \left[\frac{\lambda_{g}+\left(\lambda_{0}+\lambda_{0}^{\prime}\right)}{\left(\lambda_{0}+\lambda_{0}^{\prime}\right)} \times \frac{{ }^{40} A r}{{ }^{40 K} \mathrm{~K}}+1\right]$

Note: ${ }^{40}$ Ar refers to radiogenic ${ }^{40} \mathrm{Ar}$.

$M . Y$. refers to millions of years. 
Our Sample No. A-10492

Date Received: $\quad 9 / 22 / 93$

Your Reference: Phonecall of $12 / 3 / 93$

Date Reported: 1/20/94

Submitted By: Steven C. Bergman

ARCO Exploration and Production Technology

2300 West Plano Parkway

Plano, TX 75075

Sample Description \& Locality: Sample \# 93KAT 106A-3, volcanic sandstone

Material Analyzed: Amphibole concentrate, $-80 /+200$ mesh.

${ }^{40} \mathrm{Ar} /{ }^{40 \mathrm{~K}}=\quad .01038 \quad$ AGE $=170+/-6$ M.Y.

Argon Analyses:

$\begin{array}{ccc}{ }^{40} \mathrm{Ar}, \mathrm{ppm} & { }^{40} \mathrm{Ar} / \mathrm{Total}{ }^{\circ 0} \mathrm{Ar} & \text { Ave. }{ }^{40} \mathrm{Ar}, \mathrm{ppm} \\ .005081 & .536 & .005095 \\ .005109 & .506 & \end{array}$

Potassium Analyses:

$\begin{array}{lrr}\% \mathrm{~K} & \text { Ave. \% K } & { }^{40 \mathrm{~K}, \mathrm{ppm}} \\ 0.408 & 0.412 & 0.491 \\ 0.415 & & \end{array}$

Constants Used:

$\lambda_{\beta}=4.962 \times 10^{-10} /$ year

$\left(\lambda_{0}+\lambda_{0}^{\prime}\right)=0.581 \times 10^{-10} /$ year

${ }^{40} \mathrm{~K} / \mathrm{K}=1.193 \times 10^{-4} \mathrm{~g} / \mathrm{g}$

$\mathrm{AGE}=\frac{1}{\lambda_{\beta}+\left(\lambda_{\theta}+\lambda_{\theta}^{\prime}\right)} \ln \left[\frac{\lambda_{\beta}+\left(\lambda_{\theta}+\lambda_{\theta}^{\prime}\right)}{\left(\lambda_{\theta}+\lambda_{\theta}^{\prime}\right)} \times \frac{{ }^{40} \mathrm{Ar}}{{ }^{40} \mathrm{~K}}+1\right]$

Note: ${ }^{40}$ Ar refers to radiogenic ${ }^{40} \mathrm{Ar}$.

$M . Y$. refers to millions of years. 


\section{GEOCHRON LABORATORIES a division of} KRUEGER ENTERPRISES, INC.

\section{CONCORD AVENUE + CAMBAIDGE, MASSACHUSETTS 02138 + U.S.A} TELEPHONE: $(617) 876.3691$ TELEFAX: $(617) 661.0148$

Our Sample No. B-10448

Date Received: $9 / 22 / 93$

Your Reference: Phonecall of $12 / 3 / 93$

Date Reported: 1/6/94

Submitted By: Steven C. Bergman

ARCO Exploration and Production Technology

2300 West Plano Parkway

Plano, TX 75075

Sample Description \& Locality: Sample $93 \mathrm{KAT} 106 \mathrm{~B}-3$, volcanic sandstone

Material Analyzed: Biotite concentrate, $-80 /+200$ mesh.

${ }^{40} \mathrm{Ar} /{ }^{40 \mathrm{~K}}=\quad .01013 \quad$ AGE $=166+/-4$. M.Y.

Argon Analyses:

$\begin{array}{ccc}{ }^{40} \text { Ar, ppm } & { }^{40} \text { Ar/Total }{ }^{40} \text { Ar } & \text { Ave. }{ }^{40} \text { Ar, ppm } \\ .02599 & .547 & .02683 \\ .02773 & .628 & \\ .02677 & .567 & \end{array}$

Potassium Analyses:

$\begin{array}{lrr}\% \mathrm{~K} & \text { Ave. } \% \mathrm{~K} & 40 \mathrm{~K}, \mathrm{ppm} \\ 2.172 & 2.221 & 2.649 \\ 2.269 & & \end{array}$

Constants Used:

$\lambda_{s}=4.962 \times 10^{.10} /$ year

$\left(\lambda_{0}+\lambda_{0}^{\prime}\right)=0.581 \times 10.10 /$ year

${ }^{40} \mathrm{~K} / \mathrm{K}=1.193 \times 10.4 \mathrm{~g} / \mathrm{g}$

$A G E=\frac{1}{\lambda_{s}+\left(\lambda_{0}+\lambda_{0}^{\prime}\right)} \ln \left[\frac{\lambda_{g}+\left(\lambda_{0}+\lambda_{0}^{\prime}\right)}{\left(\lambda_{0}+\lambda_{0}^{\prime}\right)} \times \frac{{ }^{\infty} A r}{40 K}+1\right]$

Note: ${ }^{40} \mathrm{Ar}$ refers to radiogenic ${ }^{40} \mathrm{Ar}$.

M.Y. refers to millions of years. 


\section{GEOCHRON LABORATORIES a division of} KRUEGER ENTERPRISES, INC.

711 CONCORD AVENUE + CAMBRIDGE, MASSACHUSETTS O2138 + U.S.A TELEPHONE: (617) 876.3691 TELEFAX: (617) 661.0148

Our Sample No. $\quad 1-10448$

Date Received: $\quad 9 / 22 / 93$

Your Reference: Phonecall of $11 / 18 / 93$

Date Reported: $1 / 6 / 94$

Submitted By: Steven C. Bergman

ARCO Exploration and Production Technology

2300 West Plano Parkway

PIano, TX 75075

Sample Description \& Locality: Sample \# $93 \mathrm{KAT} 105 \mathrm{~B}-3$ volcanic sandstone

Material Analyzed:

Amphibole coneentrate, $-80 /+200$ mesh.

${ }^{40} \mathrm{Ar} / 40 \mathrm{~K}=$

.01062

$A G E=174+1-6$ M.Y.

Argon Analyses:

$\begin{array}{ccc}4 . \text { Ar, ppm } & { }^{40} \text { ArfTotal }{ }^{\circ} \mathrm{Ar} & \text { Ave. }{ }^{40} \mathrm{Ar}, \mathrm{ppm} \\ .004892 & .422 & .005015 \\ .005138 & .406 & \end{array}$

Potassium Analyses:
$\% \mathrm{~K}$
Ave. $\% \mathrm{~K}$
${ }^{40 \mathrm{~K}, \mathrm{ppm}}$
0.394
0.396
0.472
0.398

Constants Used:

$\lambda_{B}=4.962 \times 10.10 / y e a r$

$\left(\lambda_{0}+\lambda_{0}^{\prime}\right)=0.581 \times 10.10 /$ year

${ }^{40} \mathrm{~K} / \mathrm{K}=1.193 \times 10^{-4} \mathrm{~g} / \mathrm{g}$

$A G E=\frac{1}{\lambda_{p}+\left(\lambda_{0}+\lambda_{0}^{\prime}\right)} \ln \left[\frac{\lambda_{g}+\left(\lambda_{0}+\lambda_{0}^{\prime}\right)}{\left(\lambda_{0}+\lambda_{0}^{\prime}\right)} \times \frac{{ }_{0}{ }^{0} A r}{{ }^{40} K}+1\right]$

Note: "Ar refers to radiogenic ${ }^{\circ} \mathrm{Ar}$.

M.Y. refers to millions of years. 
To: Steve Bergman

From: Shari Kelley

Date: $3 / 21 / 94$

Subject: Procedures used for 1993 South Alaska FT samples.

Three apatite and five zircon splits from South Alaska were received on 11/1/93.

None of the zircon fractions were treated with aqua regia to remove pyrite.

The following zircon split was not dated due to insufficient zircon:
93MAT4/9 The zircons were mounted in Teflon, polished, and etched in $\mathrm{NaOH} / \mathrm{KOH}$ at $230^{\circ} \mathrm{C}$ for the times (in
hours) shown in the table below. Two of the samples contained zircon populations that had similar
etching characteristics, so only one mount was made for these 92MAT4/87, contained zircon populations that had these samples. Two samples, 93KAT106A and mounts were cut in half and each half was etched varying etching characteristics. These to attain optimum etch conditions for each population. The numt amount of time in an attempt each mount (keyed to the attached data sheets) fulation. The numbers of the grains dated in parentheses.

$\begin{array}{lcc}\text { Samole } & \text { Mount 1 } & \\ \text { 93KAT25A } & 8 & \text { Mount 2 } \\ \text { 93KAT106A } & 5 & 8 \\ & (1-4) & (5-20) \\ \text { 93KAT106B } & 7 & 8 \\ \text { 93MAT4/87 } & 7 & (1-6)\end{array}$

The zircons were placed in a reactor package with Fish Canyon zircon age standards and Corning (CNstandards and the accepted ages of the zircon standards.

the attached data sheets. The used:

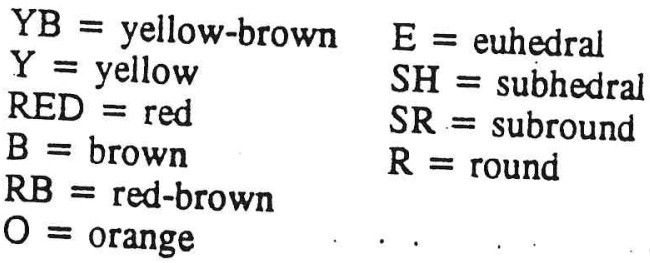

Apatite grains were mounted in epoxy on a $1 \mathrm{~cm}^{2}$ glass slide, polished to expose the grains, and etched
for 25 seconds in a $5 \mathrm{M}$ solution placed in a reactor package with Durango apatite reveal the fission tracks. The apatite samples were fission-track glass standards.

Confined track lengths were measured both in the age mounts

been irradiated for $72 \mathrm{hrs}$ at a distance of $7 \mathrm{~cm}$ from mounts and in separate grains mounts that had the order of 7.5 to $8.7 \times 10^{5}$ tracks $/ \mathrm{cm}^{2}$. 
TABLE 1. - APATITE AND ZIRCON FISSION-TRACK AGES FOR 1893 SOUTH ALASKA

\begin{tabular}{|c|c|c|c|c|c|c|c|c|c|c|}
\hline $\begin{array}{l}\text { SAMPLE } \\
\text { NUMBER }\end{array}$ & $\begin{array}{l}\text { GRAINS } \\
\text { COUNTED }\end{array}$ & $\begin{array}{c}\text { FOSSIL } \\
\text { TRACKSICM } \\
\left(\times 10^{6}\right) \\
\text { (" TAACKS) } \\
\end{array}$ & $\begin{array}{l}\text { INDUCED } \\
\text { TRACKSJCM } \\
\left(\times 10^{8}\right) \\
\text { [\# TRACKS] }\end{array}$ & $\begin{array}{c}\text { NEUTRON } \\
\text { FLUX } \\
\text { (X } 10^{16} \\
\text { NEUTRONS/CM) }\end{array}$ & $\begin{array}{l}\text { POOLED AGE } \\
\text { (STANDARD } \\
\text { ERROR) } \\
\text { (MA) }\end{array}$ & $\begin{array}{c}\text { CENTRAL AGE } \\
\text { (STANDARD } \\
\text { ERROR) } \\
\text { (MA) }\end{array}$ & $\begin{array}{l}\text { SPECTRAL } \\
\text { PEAK } \\
\text { AGE } \\
\text { (MA) }\end{array}$ & $\begin{array}{c}\text { URANIUM } \\
\text { CONTENT } \\
\text { (PPM) }\end{array}$ & $\begin{array}{l}\text { CHI } \\
\text { SQUARED } \\
\text { PROB. } \\
(\%)\end{array}$ & $\begin{array}{l}\text { MEAN } \\
\text { LENGTH } \\
\text { (S.E.)(cm) } \\
\text { [N] }\end{array}$ \\
\hline 93КАТ 108А & 20 & 0.64 & 3.01 & 1.1 & 139.2 & 130.2 & 135 & 26 & 85 & $13.4(0.2)$ \\
\hline APATITE & & 300 & 833 & $\because$ & 10 & 10.3 & & & & 85 \\
\hline 23KAT108A & 20 & 7.34 & 5.50 & 0.2 & 158.3 & 148.6 & 110 & 288 & $<1$ & \\
\hline ZIRCON & & 2503 & 975 & & 12.7 & 13.1 & & & & \\
\hline 83KAT106B & 20 & 0.45 & 2.08 & 1.1 & 139.9 & 130.9 & 130 & 18 & 20 & $13.2(0.2)$ \\
\hline APATITE & & 518 & 1206 & & 9.3 & 9.6 & & & & 100 \\
\hline 83KAT108B & 20 & 8.61 & 7.07 & 0.2 & $150.8^{\circ}$ & 140.6 & 120 & 330 & $<1$ & \\
\hline ZIRCON & & 3174 & 1304 & & 11.8 & 11.5 & & & & \\
\hline 83KAT25A & 20 & 0.46 & 1.8 .8 & $\overline{1.1}$ & $146.0^{\circ}$ & 156.0 & 80 & 16 & $<1$ & $13.3(0.3)$ \\
\hline APATITE & & 381 & 782 & & 13.2 & 16.4 & & & & 100 \\
\hline 93KAT25A & 20 & 7.10 & 6.25 & 0.2 & $142.2^{\circ}$ & 135.1 & 130 & 300 & $<1$ & \\
\hline ZIRCON & & 2157 & 037 & & 9.2 & 10.8 & & & & \\
\hline
\end{tabular}

- mean AGe 


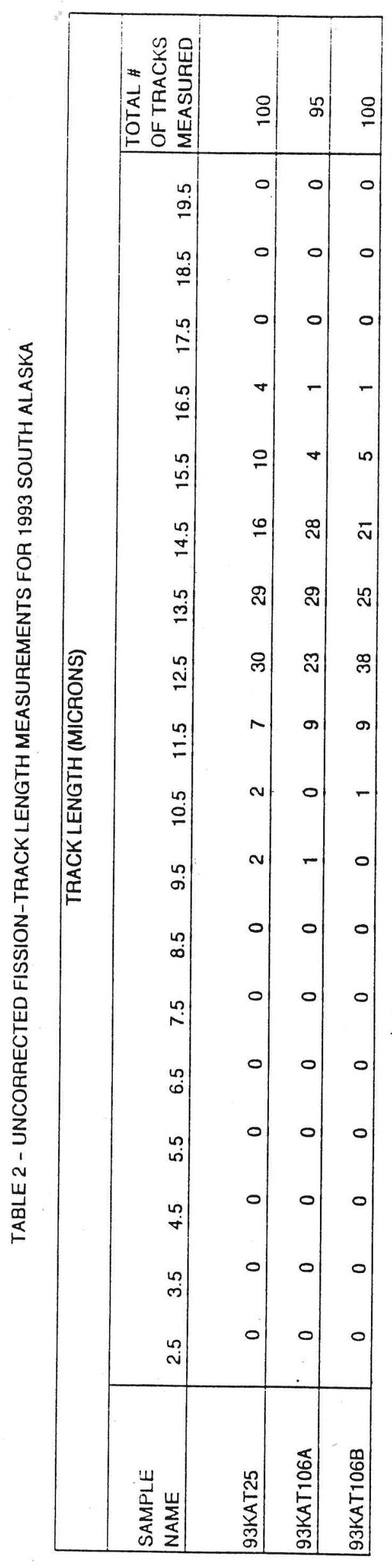




\section{FISSION TRACK AGE DATA}

\begin{tabular}{|c|c|c|c|c|c|c|c|c|c|c|}
\hline \multicolumn{2}{|c|}{ PROJECT NAME: } & \multicolumn{3}{|c|}{ SOUTH ALASKA } & DATE: & \multicolumn{2}{|l|}{$2 / 20 / 94$} & FT AGE: & \multicolumn{2}{|c|}{$139.2 \mathrm{Ma}$} \\
\hline \multicolumn{2}{|c|}{ SAMPLE NUIMBER: } & \multicolumn{3}{|l|}{ 93KAT106A } & LATTTUDE: & \multicolumn{2}{|l|}{${ }^{\circ} \mathrm{N}$} & UPPER Cl: & 161.2 & $\mathrm{Ma}$ \\
\hline \multicolumn{2}{|c|}{ LAB NUMBER: } & \multicolumn{3}{|l|}{ SMU } & LONGTUDE: & \multicolumn{2}{|l|}{$\circ \mathrm{o} W$} & LOWER Cl: & 120.2 & $\mathrm{Ma}$ \\
\hline \multicolumn{4}{|c|}{ REACTOR RUN NUMBER: RR-12-17-93Z } & $\cdot$ & \multicolumn{3}{|l|}{ ELEVATION (M): } & STD ERR: & 10.00 & $\mathrm{Ma}$ \\
\hline \multicolumn{2}{|c|}{ NEUTRON FLUX: } & \multicolumn{2}{|l|}{$1.100 E+16$} & & MICROSCOPE: & \multicolumn{2}{|l|}{ OLYMPUS } & C. COEFF: & 0.953 & \\
\hline \multicolumn{2}{|c|}{ \# SPON TRACKS (FLUX) } & \multicolumn{2}{|l|}{600} & : & \multicolumn{3}{|c|}{ MAGNIFICATION: $1250 \mathrm{X}$} & CHI: & 13.868 & 19 \\
\hline \multicolumn{2}{|c|}{ \# IND. TRACKS (FLUX) } & \multicolumn{2}{|l|}{4000} & & ROCK TYPE: & \multicolumn{2}{|l|}{ Jn } & AVG. AGE: & 135.4 & $\mathrm{Ma}$ \\
\hline NUMBER C & OF GRAINS: & 20 & & & MINERAL: & APATITE & & STD. ERR: & 7.7 & \\
\hline GRAIN \# & $\begin{array}{l}\text { AREA USED } \\
\text { (SQ. CM) }\end{array}$ & Ns & $\begin{array}{c}\text { RHO(S) } \\
\text { (/CM) }\end{array}$ & & $\mathrm{Ni}$ & $\begin{array}{l}\mathrm{RHO}(\mathrm{I}) \\
\text { (/CM) }\end{array}$ & $\begin{array}{l}\text { RATIO } \\
\text { (NS/NI) }\end{array}$ & $\begin{array}{l}\text { URANIUM } \\
\text { (PPM) }\end{array}$ & (MA) & $\begin{array}{l}\text { STD.DEV. } \\
\text { (MA) }\end{array}$ \\
\hline 1. $\mathrm{SH}$ & $2.0 \mathrm{E}-05$ & 18 & $9.000 E+05$ & & 31 & $3.100 E+06$ & 0.58 & 27.1 & 188.3 & 56.4 \\
\hline 2. SR & $2.0 E-05$ & 30 & $1.500 E+06$ & & 93 & $9.300 E+06$ & 0.32 & 81.2 & 105.3 & 22.6 \\
\hline 3. $S R$ & 4.0E-05 & 17 & $4.250 E+05$ & & 34 & $1.700 E+06$ & 0.50 & 14.8 & 162.5 & 48.8 \\
\hline 4. $R$ & 2.0E-05 & 18 & $9.000 E+05$ & & 42 & $4.200 E+06$ & 0.43 & 36.7 & 139.5 & 39.8 \\
\hline 5. SR & $2.0 E-05$ & 10 & $5.000 E+05$ & & 18 & $1.800 E+06$ & 0.56 & 15.7 & 180.3 & 71.5 \\
\hline 6. $R$ & $4.0 E-05$ & 47 & $1.175 E+06$ & & 81 & $4.050 E+06$ & 0.58 & 35.3 & 188.2 & 35.5 \\
\hline 7. $\mathrm{SH}$ & $2.0 E-05$ & 1 & $5.000 E+04$ & & 4 & $4.000 E+05$ & 0.25 & 3.5 & 81.7 & 91.5 \\
\hline 8. SR & 2.0E-05 & 28 & $1.400 E+06$ & & 76 & $7.600 E+06$ & 0.37 & 66.3 & 120.1 & 27.1 \\
\hline 9. $\mathrm{SH}$ & $2.0 \mathrm{E}-05$ & 7 & $3.500 E+05$ & & 16 & $1.600 E+06$ & 0.44 & 14.0 & 142.4 & 64.8 \\
\hline 10. SR & $2.0 \mathrm{E}-05$ & 13 & $6.500 E+05$ & & 56 & $5.600 E+06$ & 0.23 & 48.9 & 75.9 & 23.6 \\
\hline 11. SA & $4.0 E-05$ & 5 & $1.250 E+05$ & & 17 & $8.500 E+05$ & 0.29 & 7.4 & 96.1 & 49.1 \\
\hline 12. SH & 4.0E-05 & 5 & $1.250 E+05$ & & 11 & $5.500 E+05$ & 0.45 & 4.8 & 147.9 & 80.0 \\
\hline 13. SH & $4.0 \mathrm{E}-05$ & 6 & $1.500 E+05$ & & 23 & $1.150 E+06$ & 0.26 & 10.0 & 85.3 & 39.3 \\
\hline 14. SR & 4.0E-05 & 36 & $9.000 E+05$ & & 81 & $4.050 E+06$ & 0.44 & 35.3 & 144.6 & 29.7 \\
\hline 15. SR & 2.0E-05 & 19 & $9.500 E+05$ & & 42 & $4.200 E+06$ & 0.45 & 36.7 & 147.2 & 41.2 \\
\hline 16. SR & $2.0 E-05$ & 3 & $1.500 E+05$ & & 8 & $8.000 E+05$ & 0.38 & 7.0 & 122.2 & 82.9 \\
\hline 17. SR & $2.0 E-05$ & 19 & $9.500 E+05$ & & 44 & $4.400 E+06$ & 0.43 & 38.4 & 140.6 & 39.1 \\
\hline 18. SR & $4.0 E-05$ & 40 & $1.000 E+06$ & & 79 & $3.950 E+06$ & 0.51 & 34.5 & 164.5 & 32.7 \\
\hline 19. SH & $4.0 E-05$ & 123 & $3.000 E+05$ & & 30 & $1.500 E+06$ & 0.40 & 13.1 & 130.3 & 44.9 \\
\hline 20. SH & $8.0 E-05$ & $65 \varepsilon$ & $8.125 E+05$ & & 147 & $3.675 E+06$ & 0.44 & 32.1 & 143.9 & $22: 3$ \\
\hline & $6.2 E-0.4$ & 3996 & $6.435 E+05$ & & 933 & $3.010 E+06$ & 0.43 & 26.3 & & \\
\hline
\end{tabular}




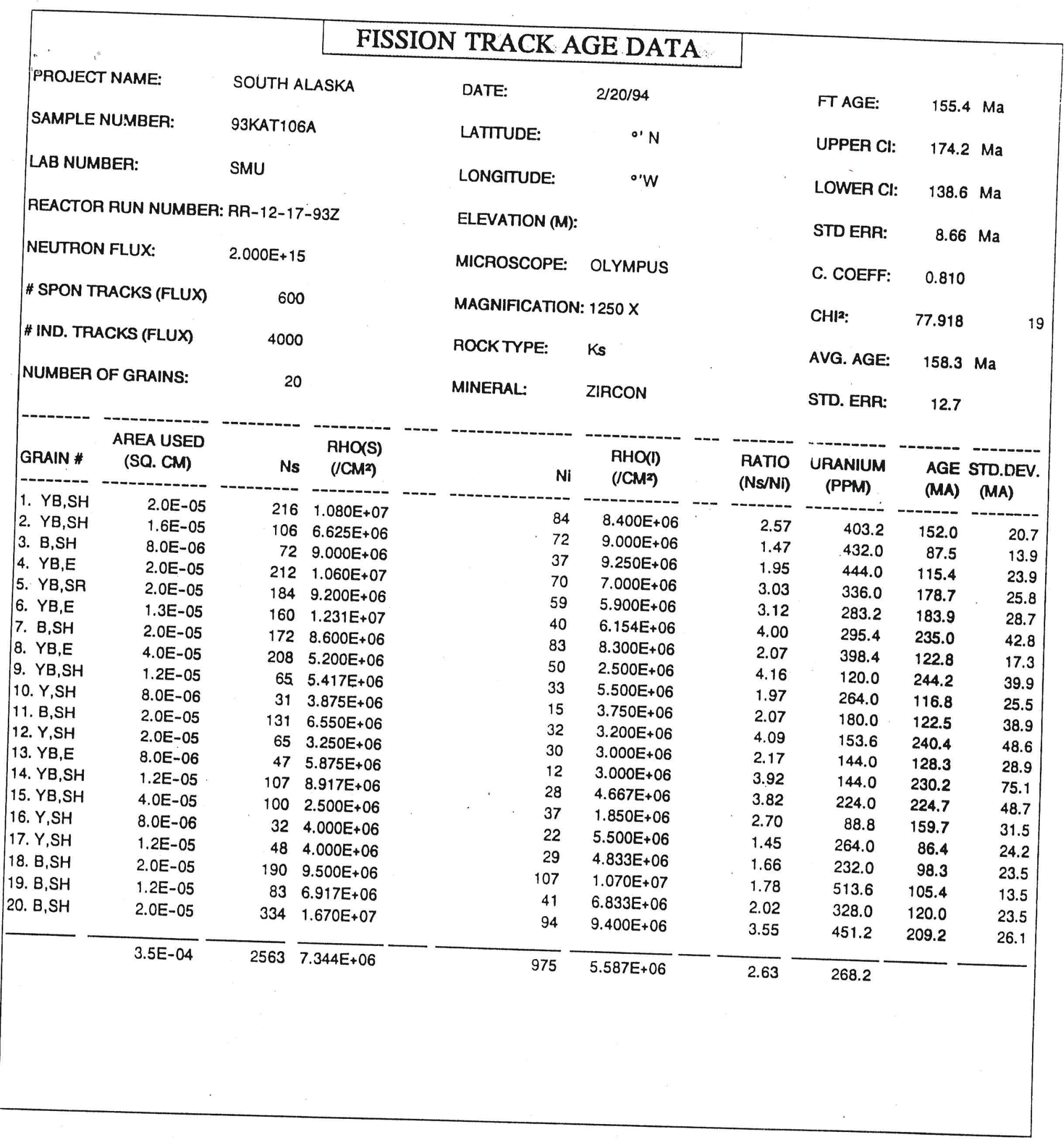



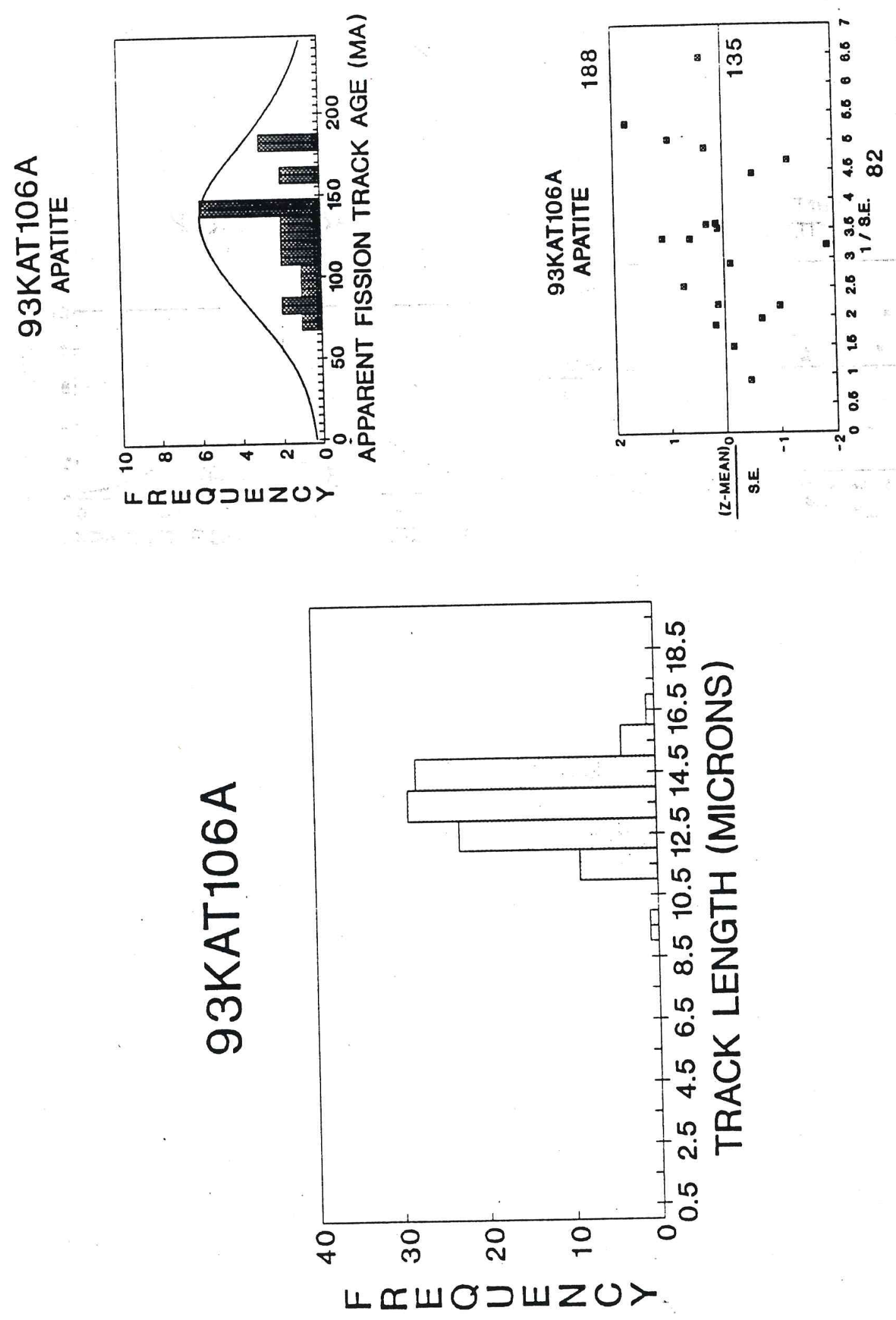

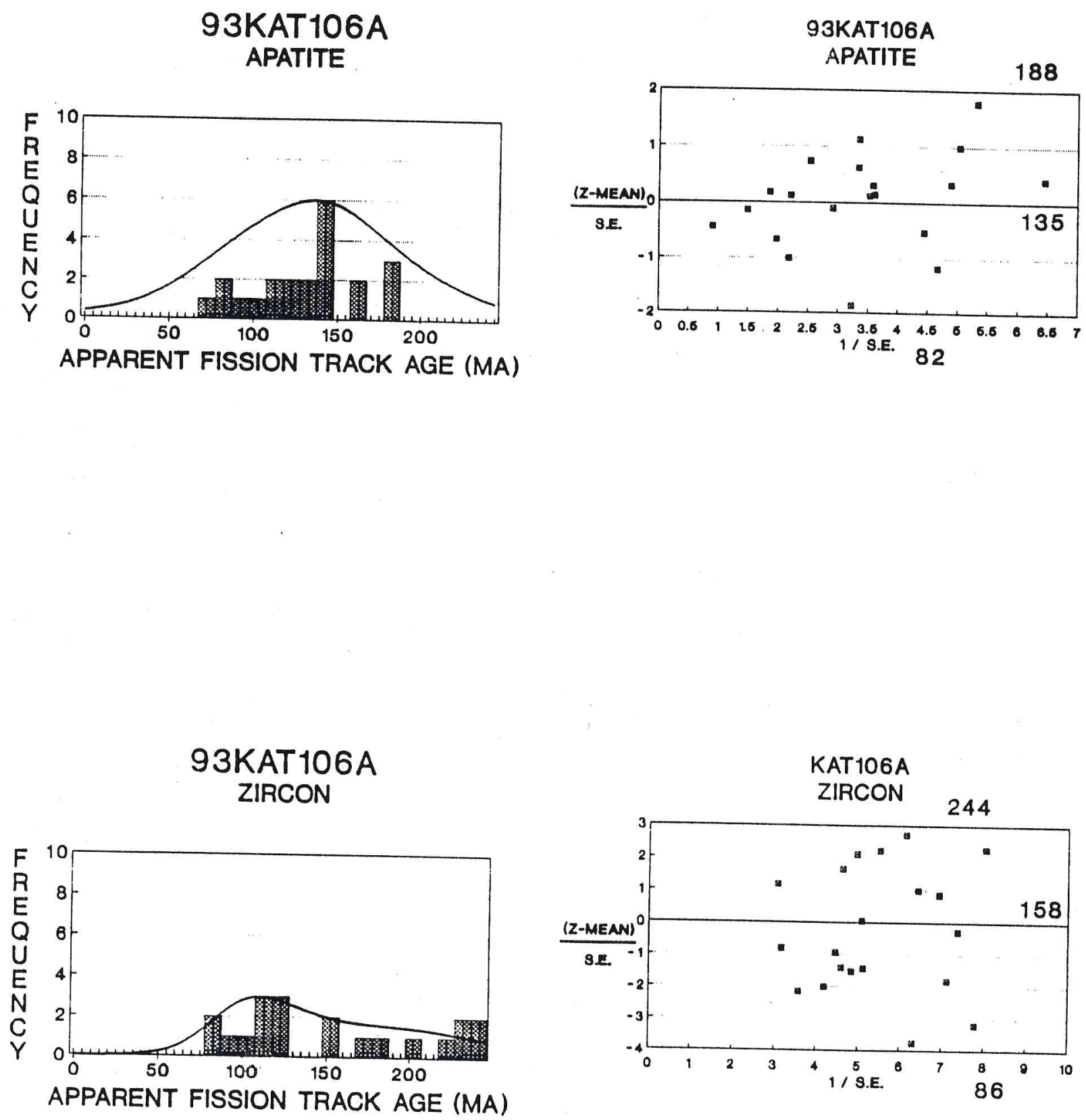
FISSION TRACK AGE DATA

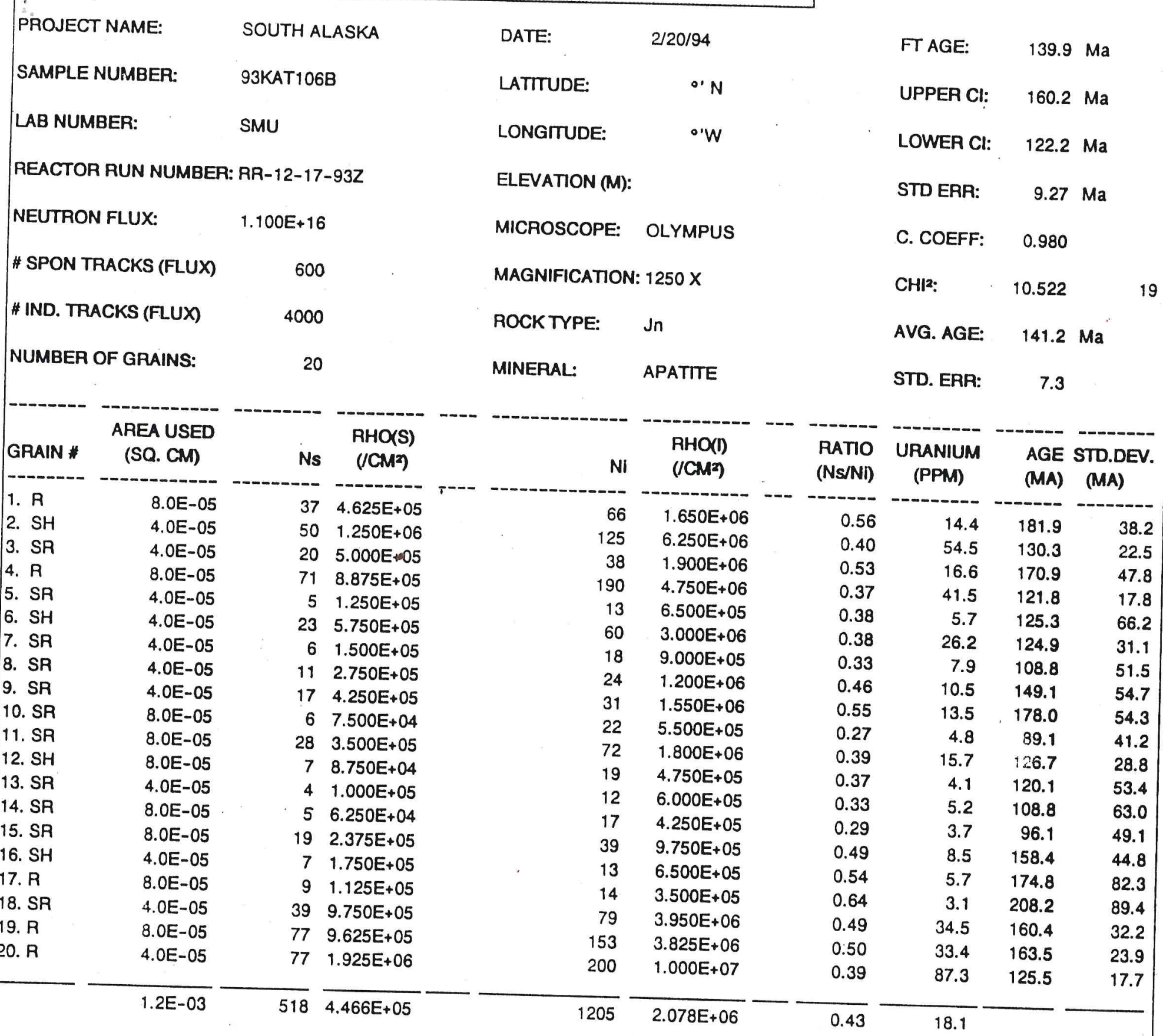




\section{FISSION TRACK AGE DATA}

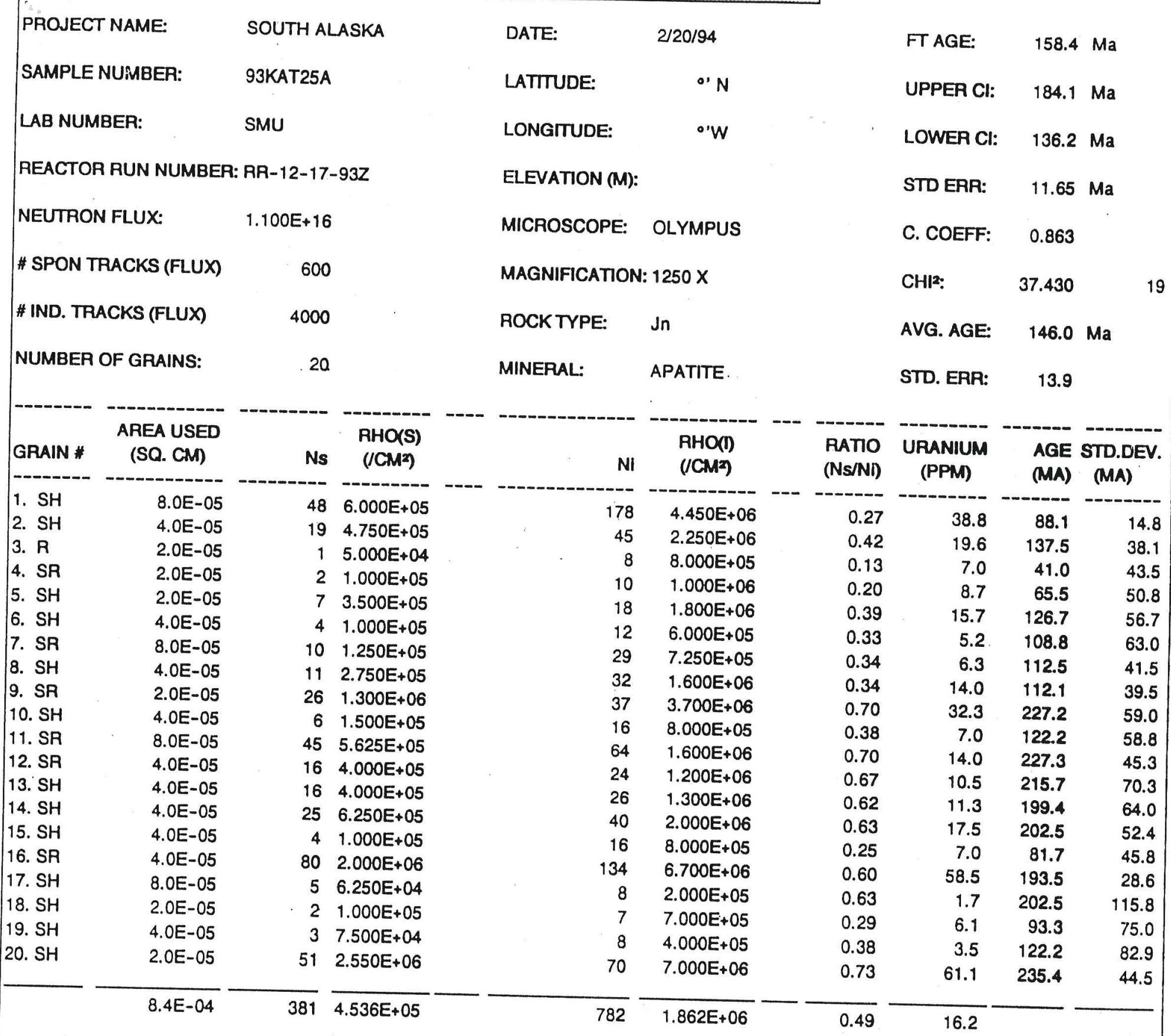



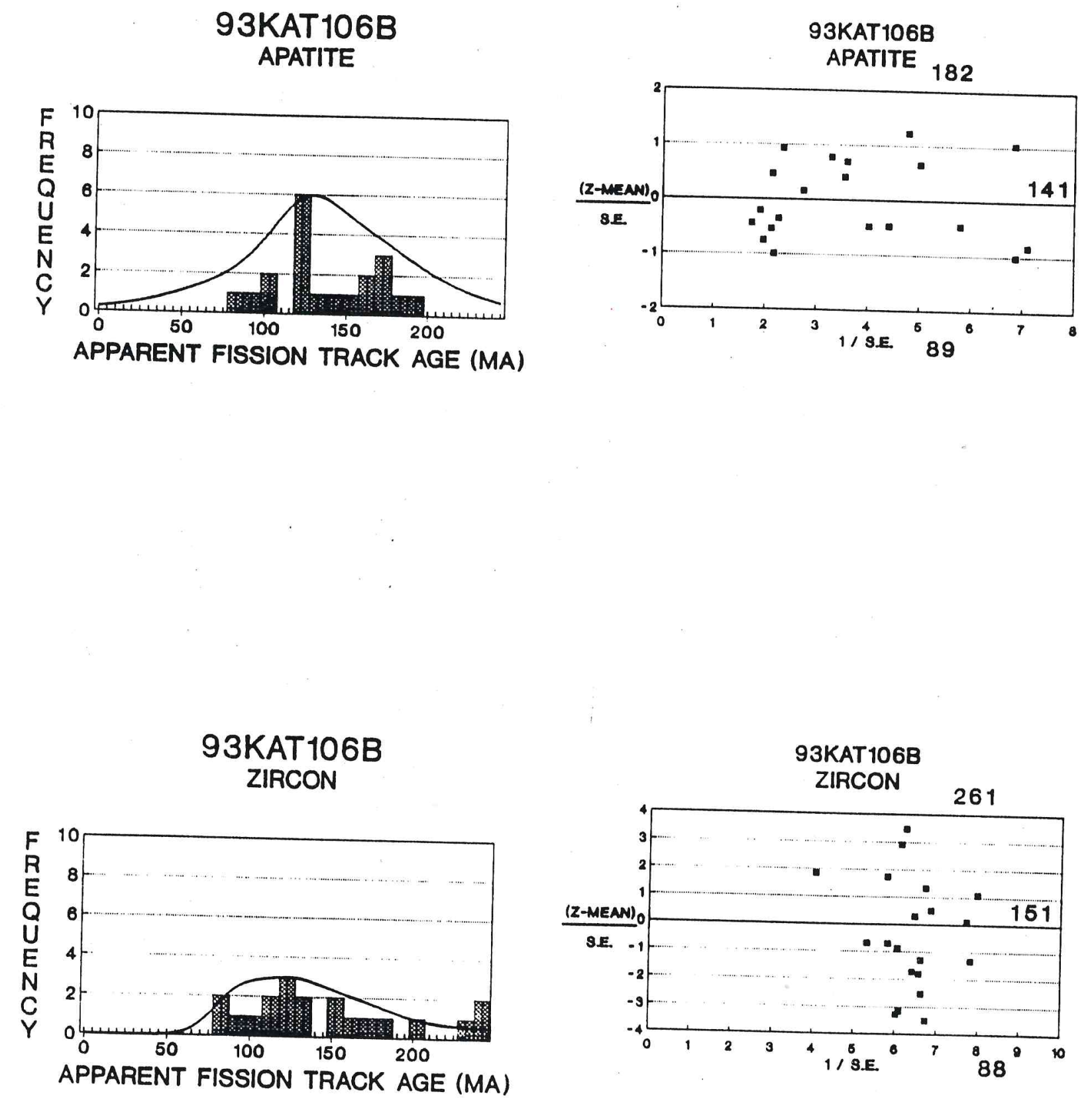

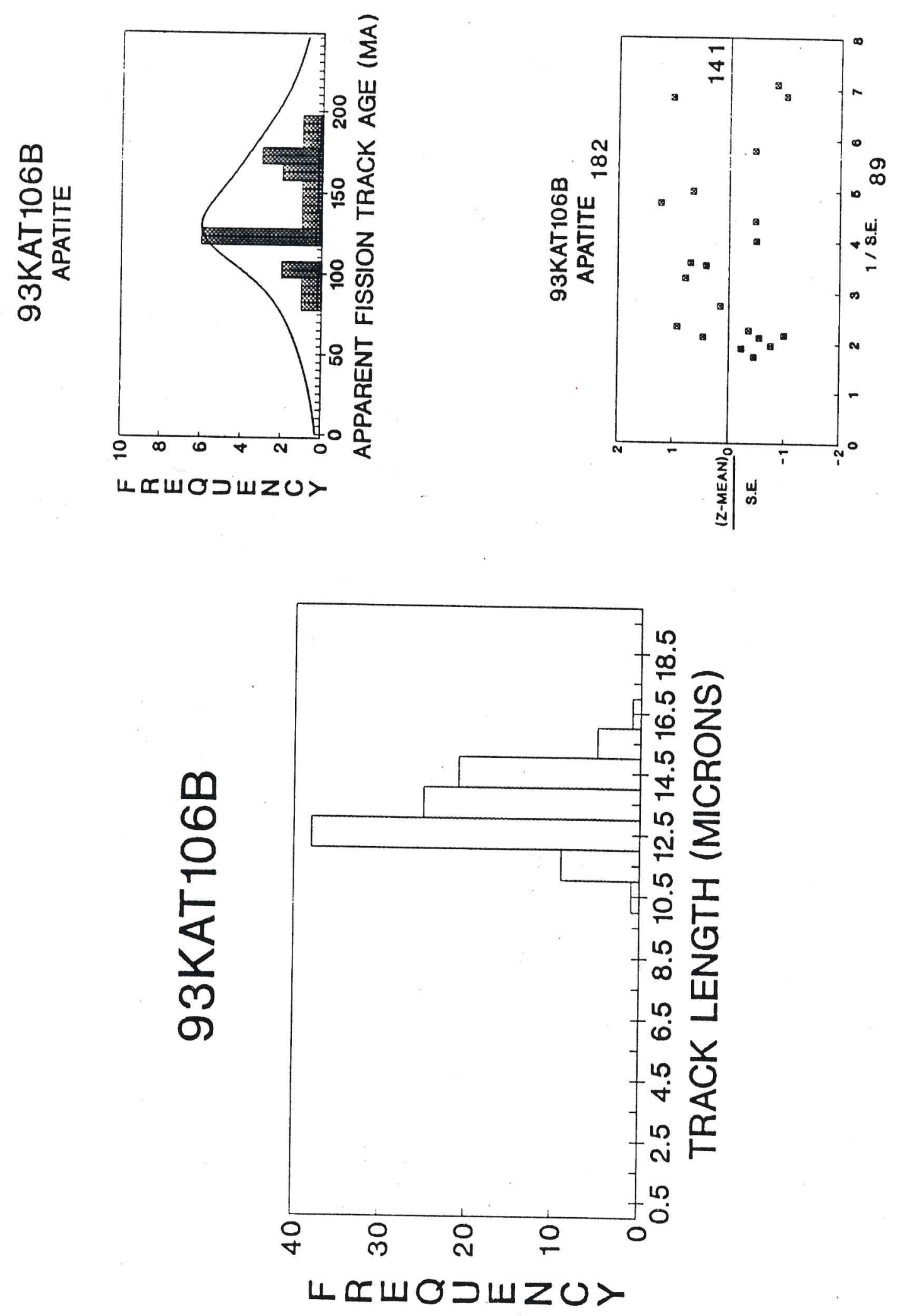
FISSION TRACK AGE DATA

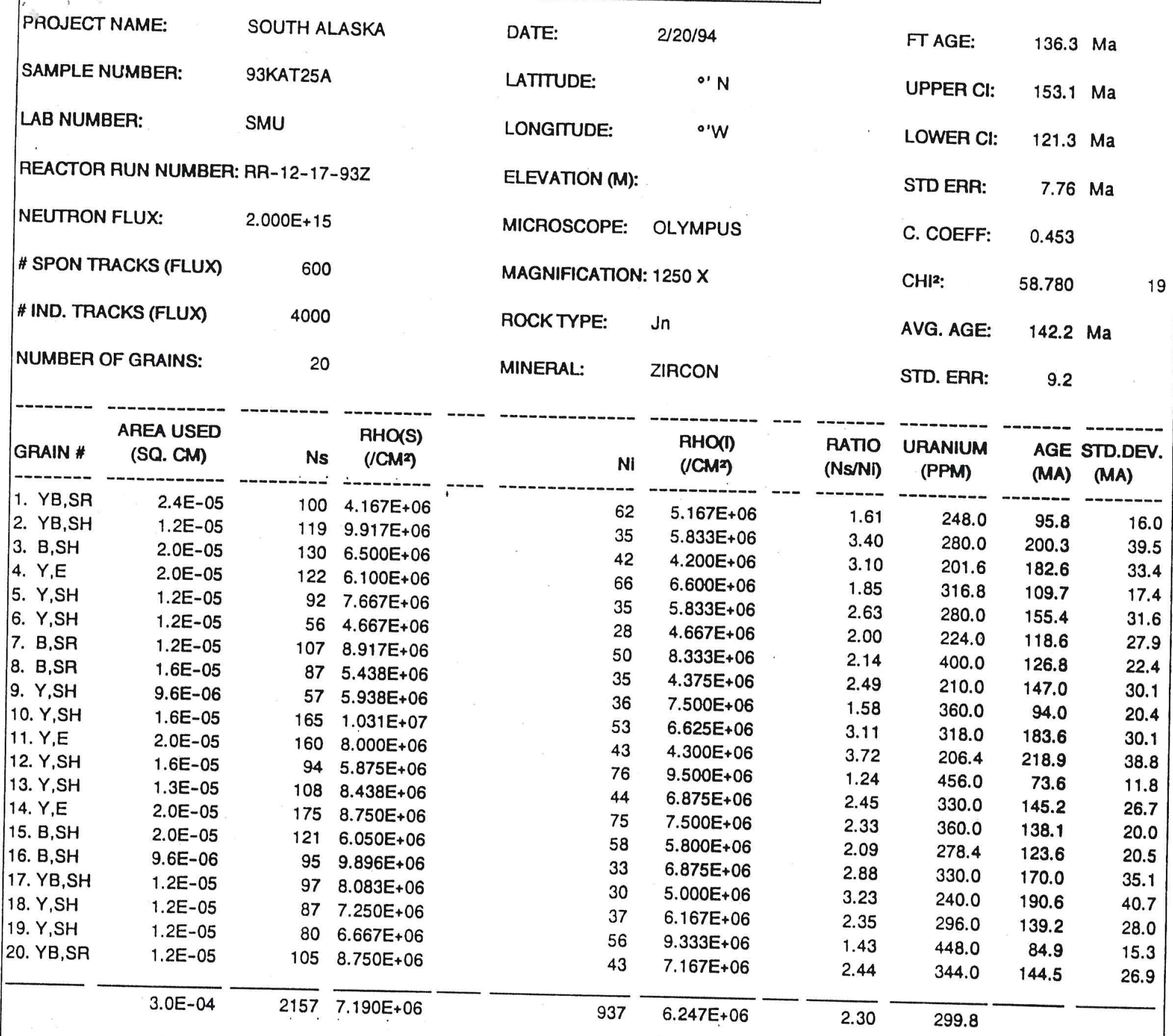



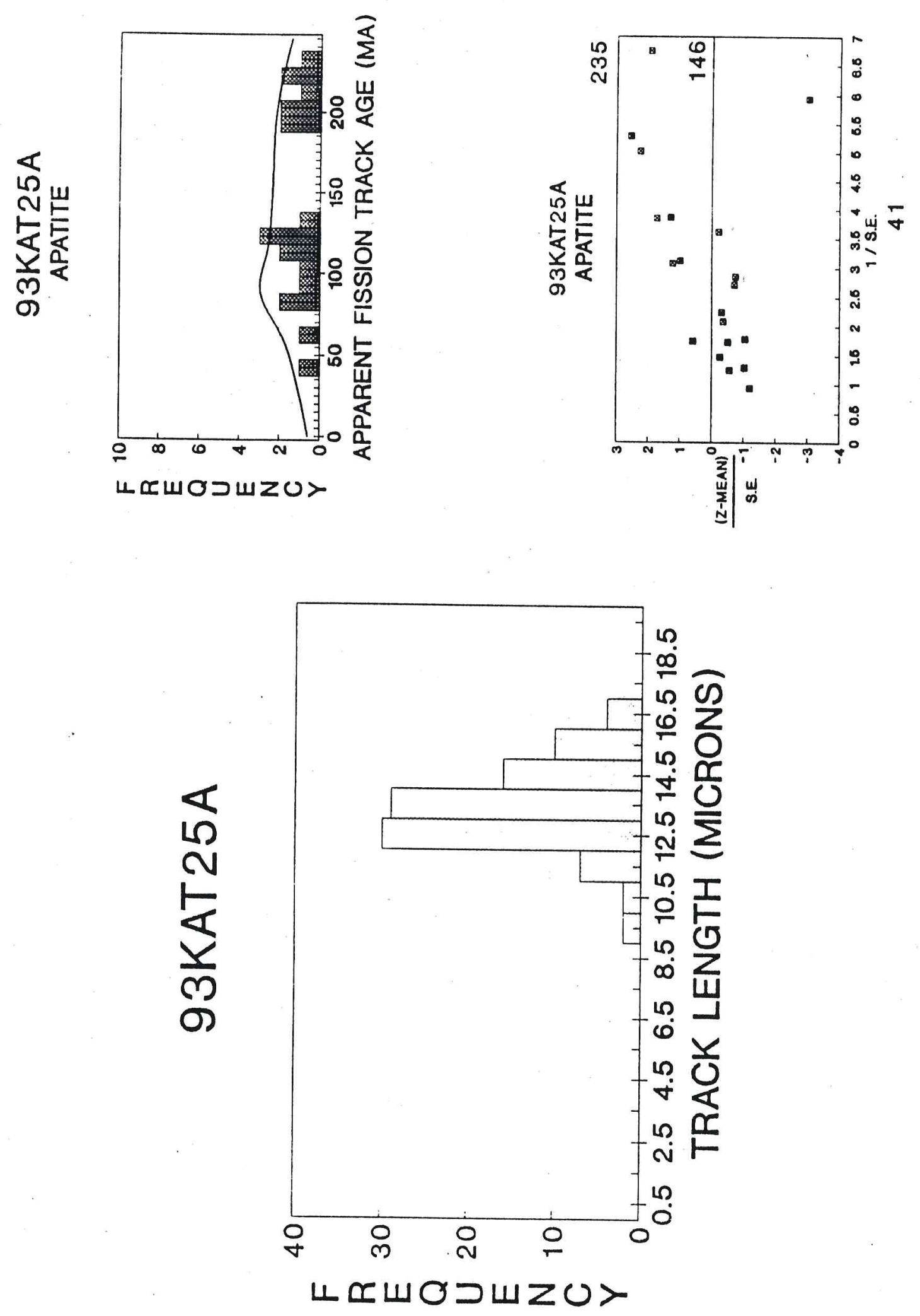

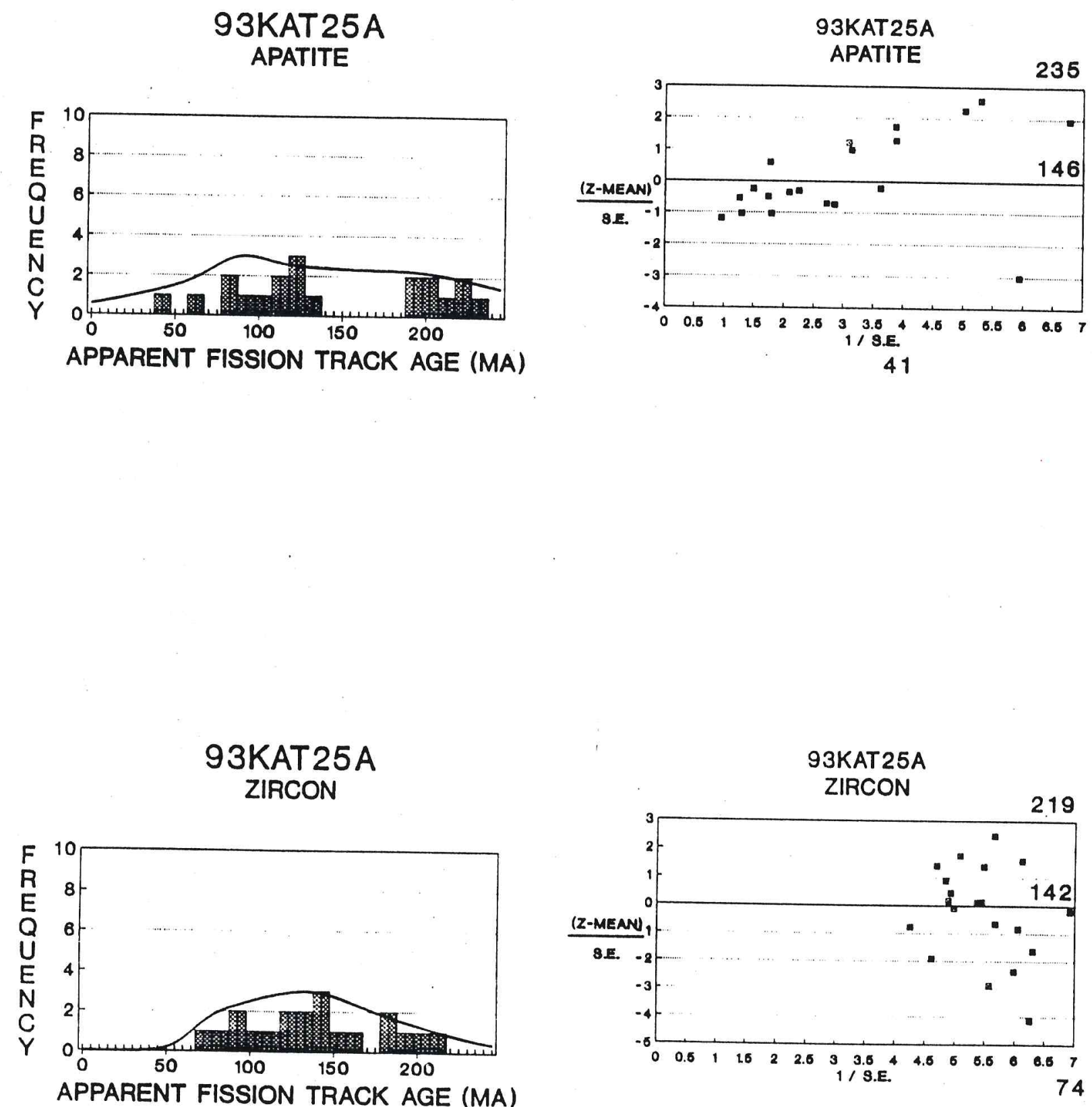